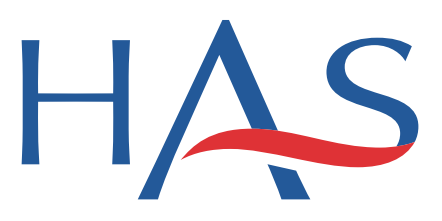

hAUTE AUTORITÉ DE SANTÉ

\title{
Rapport de mission
}

État de l'art (national et international) en matière de pratiques de simulation dans le domaine de la santé

Dans le cadre du développement professionnel continu (DPC) et de la prévention des risques associés aux soins 


\section{Le Pr Jean-Claude Granry et le Dr Marie-Christine Moll sont les auteurs de ce rapport}

Le traitement des bases de données a été réalisé par Virginie Roue

Ce rapport, comme l'ensemble des publications, est téléchargeable sur:

www.has-sante.fr 


\section{Sommaire}

Liste des abréviations 4

Lettre de mission HAS

Synthèse du rapport $\quad 7$

1. Définition de la simulation 7

2. Enseignements de l'utilisation de la simulation dans les activités à risques en dehors de la santé 7

3. Synthèse de l'état des lieux international : Amérique du Nord et Europe 8

4. Intérêts pédagogiques de la simulation en santé 9

5. Simulation, gestion des risques et DPC 10

Conclusion et propositions 11

Introduction 13

1. Définitions de la simulation 13

2. Historique de la simulation en santé 15

3. Simulation en dehors de la santé 16

4. Simulation en santé : état des lieux 26

5. Simulation en santé : techniques, bonnes pratiques et perspectives 57

6. Conclusion : intérêts et limites de la simulation en santé - propositions 78

Annexe 1

Annexe 2

Annexe 3

Annexe 4

Annexe 4b 88

Annexe 5

Annexe 5b 93

Annexe 6

Annexe 7

Annexe 8

Annexe 9 


\section{Liste des abréviations}

ACRM

ABSM

ACGME

AFSARMU

ALS

EDF

AVS

CAO

CESU

$\mathrm{CH}$

$\mathrm{CHU}$

CLCC

$\mathrm{CMO}$

CRM

DPC

DU

ENAC

ENSM

EPP

ESPIC

FAP

FMC

HF

HPST

IFCS

IFSI

LOFT

MOCA

$\mathrm{OACl}$

OMI

QT

$\mathrm{PSPH}$

SAMU

SESAM
Anesthesia Crisis Ressource Management

American Board of Medical Specialities

Accreditation Concil for Graduate Medical Education

Association Francophone de Simulation en Anesthésie Réanimation et en Médecine d'Urgence

Advanced Life Simulation

Électricité de France

Advence Video Systeme

Conception Assistée par Ordinateur

Centre d'Enseignement des Soins d'Urgence

Centre Hospitalier

Centre Hospitalier Universitaire

Centre de Lutte Contre le Cancer

Chief Medical Officer

Crew Resources Management

Développement Professionnel Continu

Diplôme d'Université

École Nationale de l'Aviation Civile

École Nationale Supérieure Maritime

Évaluation des Pratiques Professionnelles

Établissement de Santé Privé d'Intérêt Collectif

Fiche d'Aide à la Progression

Formation Médicale Continue

Haute-Fidélité

Hôpital Patient Santé Territoire

Institut de Formation des Cadres de Santé

Institut de Formation en Soins Infirmiers

Line Oriented Flight Training

Maintenance Of Certification in Anesthesia

Organisation de l'Aviation Civile Internationale

Organisation Maritime Internationale

Qualification de Type

Participant au Service Public Hospitalier

Service d'Aide Médicale d'Urgence

Society in Europe for Simulation Applied to Medicine

Simulateur BF Simulateur Basse-Fidélité

Simulateur HF Simulateur Haute-Fidélité 


\section{Lettre de mission HAS}

\section{HAS}

HAUTE AUTORITÉ DE SANTE

\section{LE PRESIDENT}

Monsieur le Professeur Jean-Claude GRANRY Madame le docteur Marie-Christine MOLL $\mathrm{CHU}$ d'Angers

4 , rue Larrey

49933 ANGERS cedex 9

Saint-Denis, le 2 février 2010

DAQSS

Service Évaluation et amélioration des pratiques

Nos réf. : 2010-0019

Dossier suivi par Frédérique POTHIER

Objet: Lettre de mission

Madame,

Monsieur le Professeur,

A l'instar des simulateurs de vol dans l'aéronautique, l'enseignement virtuel est entré dans le domaine de la santé. Au-delà du volet formation initiale, la structuration de plateformes de mise en situation simulée, avec ou sans environnement technologique dédié, peut s'inscrire idéalement dans le déploiement de programmes de développement professionnel continu (DPC). II peut aussi constituer un moyen de progresser dans la gestion des risques liés aux soins et des incidents critiques.

A cet égard, la HAS souhaite confier vous une mission afin de réaliser un état des lieux des initiatives ou expériences en cours, en France et à l'échelon international, en centrant les travaux sur le volet du développement professionnel continu, et de formuler des recommandations susceptibles de favoriser le déploiement de ce type de plateforme et de faciliter le partage de bonnes règles de fonctionnement.

Une attention particulière devra être accordée au strict respect du principe d'indépendance professionnelle.

Les modalités de réalisation de la mission ainsi que son calendrier prévisionnel sont définis dans le document de cadrage ci-joint et adopté par le Collège de la HAS lors de la séance du 9 décembre 2009 .

Dans l'attente de vous rencontrer, nous restons à votre disposition pour toute information qui pourrait vous être nécessaire et vous prions d'agréer, Madame, Monsieur le Professeur, Texpression de nos salutations distinguées.

PJ : mentionnée.

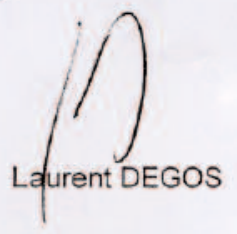

Secretariat: Chantal Dalencour

Tél +33(0) 155937388 - Fax $+33(0) 155937390$ c.dalencourt@has-sante.ft

2 avenue du Stade de France - F 93218 Saint-Denis La Plaine CEDEX - Tel.; $+33(0) 155937000-F a x ;+33(0) 155937400$ www.has-5ante.fr $N^{0}$ SIRET : 11000044500012 - COde APE: 751 A 



\section{Synthèse du rapport « Simulation »}

Simulation et Développement Professionnel Continu (DPC) sont les deux concepts qui ont guidé la rédaction de ce travail.

Dans un premier temps, il nous a paru important de définir la notion de "Simulation » dans ce contexte puis, après un bref rappel historique, d'étudier l'apport de la simulation pour la formation " continue » dans certains métiers à risques. L'expérience de ces métiers nous est apparue indispensable à connaître comme préalable à l'étude de la simulation en santé.

Celle-ci fait ensuite l'objet de l'état des pratiques internationales, tant à travers la littérature (enquêtes et revues) que par la vision de spécialistes reconnus. La visite de certains centres de référence a permis de préciser la réalité de ces pratiques sur le terrain.

La méthodologie et les résultats de deux enquêtes nationales effectuées dans le cadre de la mission sont rapportés. La première est une enquête " généraliste " utilisant un questionnaire simplifié et destinée à appréhender une vision globale de l'utilisation des techniques de simulation au sein des établissements de santé et des écoles des métiers de la santé (médecins, infirmières) en France. La seconde, plus complète, a pour but de préciser l'organisation et le fonctionnement des centres de simulation installés ou en cours d'installation.

La dernière partie du rapport développe les aspects actuels de la formation par la simulation. Les techniques de simulation sont décrites, tandis qu'une revue de la littérature retient les points essentiels de la formation par cette méthode. Le lien est ainsi établi avec le développement professionnel continu, au cours duquel la simulation peut représenter un outil particulièrement efficace.

Au terme de ce travail, des propositions générales sur la simulation en santé sont présentées.

\section{Définition de la simulation}

La définition suivante nous a paru intéressante, car elle intègre bien le rôle de la simulation dans la formation des professionnels de santé : « Le terme Simulation en santé correspond à l'utilisation d'un matériel (comme un mannequin ou un simulateur procédural), de la réalité virtuelle ou d'un patient standardisé pour reproduire des situations ou des environnements de soin, dans le but d'enseigner des procédures diagnostiques et thérapeutiques et de répéter des processus, des concepts médicaux ou des prises de décision par un professionnel de santé ou une équipe de professionnels.» ${ }^{1}$

\section{Enseignements de l'utilisation de la simulation dans les activités à risques en dehors de la santé}

La mise en place de la formation par la simulation dans les industries à risques, que l'on considère aujourd'hui comme activités ultra sûres, a répondu à deux motivations.

La première est la conviction profonde que la simulation pouvait apporter une amélioration de la sécurité. Ce concept, qui n'a pu être aujourd'hui démontré, reste cependant pour ces secteurs une évidence sur laquelle on ne peut revenir.

Le deuxième élément, qui a motivé la mise en place, est l'enjeu économique. En effet, il est moins coûteux d'entraîner des pilotes sur des matériels à terre que de faire voler un avion de ligne à vide. Concernant l'industrie nucléaire française, des gains financiers ont même été mesurés par une diminution de $20 \%$ de la fréquence des arrêts automatiques des réacteurs.

1. Chambre des représentants USA, 111th congress 02-2009. 
D'autres points communs méritent d'être retenus. En premier lieu, la formation par la simulation a d'abord été utilisée en formation initiale ou continue avec des moyens technologiques (simulateurs, mannequins, etc.). Elle s'est ensuite intéressée aux comportements des professionnels puis à la validation de leurs compétences. Les facteurs humains, liés aux activités en équipes, sont entrés progressivement dans le champ de la simulation, car il a été démontré qu'ils sont un des éléments prépondérants de la sécurité dans ces secteurs d'activité. Enfin, dans tous les cas, des investissements très importants ont été réalisés pour la conception et l'achat de simulateurs « pleine échelle » - reproduisant très fidèlement l'environnement de travail -, et pour le dimensionnement des besoins en ressources humaines, nécessaires à la formation des professionnels (formateurs compétents, temps prévus dans les effectifs en vue de la formation). Investissement et caractère obligatoire de ces formations reflètent une véritable politique institutionnelle reposant sur des bases sécuritaires (les accidents deviennent inacceptables pour le public) et économiques.

L'amélioration du savoir-faire grâce à la simulation est indiscutable de même que l'analyse et la modification des comportements, tout particulièrement en situation de crise. En revanche, certaines questions demeurent sans réponse : la formation par simulation diminue-t-elle les risques aéronautiques ou les incidents dans les centrales nucléaires ? Existe-t-il une corrélation entre formation par cette méthode et la performance ? Est-il justifié d'évaluer les professionnels par ces techniques?

\section{Synthèse de l'état des lieux international : Amérique du Nord et Europe}

\section{A. En Amérique du Nord : la simulation est un outil pédagogique à part entière}

Elle est utilisée de manière routinière dans l'enseignement initial des professions médicales et paramédicales. Une distinction existe entre programme de simulation et centre de simulation (lieu où se déroule la formation). La formation par simulation représente un argument d'attractivité des établissements de santé. L'organisation des centres se fait en réseau pour optimiser les ressources. La simulation est utilisée pour la certification ou la recertification des professionnels de santé et enfin, une accréditation des centres est possible au moyen de référentiels validés (1).

\section{B. En Europe, la simulation est un outil pédagogique bien implanté, mais les inégalités sont importantes}

On y constate un développement plus récent qu'en Amérique du Nord. L'investissement de départ est néanmoins approprié (locaux, ressources humaines, matériel) pour la majorité des centres. Une part importante des investissements revient à un financement privé et à la communauté européenne. L'activité de formation professionnelle continue occupe une place prépondérante où toutes les disciplines sont envisagées, avec une ouverture à d'autres secteurs que la santé. Enfin, l'activité de recherche en simulation est croissante.

\section{En France, la simulation est une activité émergente, mais qui intéresse de plus en plus le monde de la santé}

L'activité de simulation en santé, même si elle n'est pas encore très dense, se répartit sur l'ensemble du territoire national et intéresse toutes les disciplines. Beaucoup d'activités utilisant les principes de la simulation existent, mais demeurent assez artisanales.

Les centres français de simulation disposent en général de locaux dédiés de superficie modeste (en moyenne moins de trois salles par centre, équipées ou non de systèmes vidéo). Les locaux annexes permettant convivialité et debriefing sont peu développés. À l'exception de rares établissements, les matériels et équipements sont peu nombreux et encore assez peu diversifiés (tous déclarent cependant utiliser au moins un mannequin haute-fidélité). Par exemple, les simulateurs chirurgicaux sont rarement cités au sein des centres constitués. La simulation, ayant recours à des environnements virtuels, est quasi inexistante. 
Beaucoup de thèmes ou de disciplines sont couverts par la simulation même si certains sont plus récurrents, tels que ceux se rapportant à l'anesthésie-réanimation, à la médecine d'urgence et à la périnatalité (néonatalogie et obstétrique) de même que tout ce qui concerne les soins infirmiers.

Les ressources humaines allouées sont faibles et particulièrement pour les personnels supports (technicien, hôtesses et secrétaires). En revanche, il existe une réelle compétence au sein des centres puisqu'ils disposent le plus souvent de formateurs formés à la simulation (formations diplômantes).

La qualité des réponses concernant les ressources humaines montre une difficulté à évaluer précisément le niveau de celles-ci, souvent intriquées avec les personnels des services de soins des établissements. II est probable qu'une part non négligeable de l'activité soit réalisée sur du temps non rémunéré (temps personnel, repos de garde, etc.). De la même manière que pour les ressources humaines, les structures semblent mal définies, les organigrammes et la gouvernance sont peu ou pas formalisés. Les structures présentent une grande disparité en matière d'organisation et de modalités de formation dispensées. II est par ailleurs difficile d'évaluer le niveau d'activité moyen des centres.

Les centres souffrent d'un manque de règles ou de bonnes pratiques (par exemple les résultats de la séance font rarement l'objet d'une fiche d'aide à la progression), d'une grande dispersion des méthodes, des moyens, et des tarifs de formation. Et enfin, contrairement à ce qui est observé dans le reste de l'Europe, la recherche est très peu structurée.

\section{Intérêt pédagogique de la simulation en santé}

Plusieurs revues de la littérature ont été réalisées concernant ce sujet. Parmi elles, on peut citer par exemple la BEME Systematic review (2) qui a étudié cinq bases de données par l'intermédiaire de quatre-vingt-onze mots-clés sur une période de trente-cinq ans (1969-2003). Durant cette période, les auteurs ont retenu cent neuf études selon les quatre critères suivants : élimination des articles réalisés avec une méthodologie empirique ; utilisation d'un simulateur pour une évaluation objective des apprenants avec des résultats quantitatifs ; travaux de recherche comparatifs, expérimentaux ou quasi expérimentaux ; travaux de recherche impliquant la simulation comme unique méthode de formation. Les données des cent neuf articles ont été analysées par neuf lecteurs indépendants, selon un protocole standardisé.

Les résultats rapportés tiennent compte essentiellement des implications de ces travaux pour la pratique et selon les principes de l'Evidence Based Medicine. Ils peuvent être résumés ainsi :

- le debriefing est une étape essentielle de la simulation (47\% des articles retenus). De même que la pratique répétée ( $39 \%$ des articles) ;

- l'intégration de la simulation dans le curriculum global de formation (initiale ou continue) est un autre point majeur ( $25 \%$ des articles cités) ;

- la formation doit être réalisée avec des niveaux de difficulté croissants (14\%) en utilisant plusieurs stratégies d'apprentissage $(10 \%)$;

- la formation doit permettre de reproduire des situations cliniques variées (10\% dans un environnement contrôlé (où les apprenants peuvent faire, détecter ou corriger des erreurs sans risque) $(9 \%)$;

- les expériences pédagogiques avec la simulation doivent être reproductibles, standardisées et impliquant activement les participants ( $9 \%$ des articles) ;

- les objectifs pédagogiques doivent être précis et explicites, permettant des comparaisons et des résultats mesurables $(6 \%)$;

- enfin, il convient de s'assurer que le simulateur est un outil validé d'apprentissage (3\%). 


\section{Simulation, gestion des risques et DPC}

\section{A. Prérequis utilisés}

Les projets de DPC s'appuieront sur la définition qu'en fait la loi (la loi HPST2 réunit la formation continue et l'EPP sous le terme générique de DPC), et engloberont à la fois le champ de la qualité et de la gestion des risques. Les caractéristiques validant les projets ont été définies comme suit :

- une analyse de pratique en lien avec l'activité du professionnel concerné ;

- un enjeu d'amélioration de la qualité permettant de dégager une marge significative de progrès ainsi que la mesure d'un résultat qui signe l'amélioration effective de la pratique ;

- la prise en compte de références validées et d'un outil entériné par la HAS.

Par ailleurs, en ce qui concerne la gestion des risques et le DPC, des pratiques de " fiabilités humaines » ont été développées dans les industries dites ultra sûres. Les erreurs, étant surtout liées aux facteurs humains, il est nécessaire de développer des stratégies pour les récupérer ou les anticiper. Ces pratiques de fiabilité ou attitudes sécuritaires peuvent être détectées en simulation, et même servir de fil conducteur à l'analyse du scénario en termes de gestion des risques. Aussi, plusieurs approches par la simulation ont été abordées : la fiabilisation des pratiques par la simulation des accidents afin d'encourager l'apprentissage par l'erreur (RMM simulée), mais aussi le développement chez les professionnels de la capacité de détection et de récupération des erreurs en utilisant la synergie d'équipe et les attitudes sécuritaires.

\section{B. Programmes de DPC, les outils utilisables}

L'évaluation des pratiques emprunte à la qualité et à la gestion des risques de nombreux outils et, par ailleurs, se déploie selon des approches de type amélioration de la qualité ou gestion des risques. Un parallèle est établi entre les outils HAS existants et leur déclinaison en méthodes de simulation. Un mode d'emploi est proposé pour la mise en œuvre de programmes de DPC, et des exemples l'illustrent (programme de DPC pour la simulation de consultation d'annonce du cancer ou pour la check-list au bloc opératoire).

L'objectif de mise en place de programmes consiste à s'assurer que chaque professionnel de santé dispose d'une stratégie visant l'amélioration continue et l'évaluation périodique de ses pratiques.

Cette stratégie peut être de nature individuelle ou s'intégrer dans une démarche plus globale d'équipe, de service, de pôle ou de discipline nécessitant l'implication du management dans le domaine de l'amélioration des pratiques. Il est bien évident que la simulation ne peut constituer à elle seule un programme de DPC. C'est bien la combinaison des outils et des approches individuelles, collectives et de différents modes de formation qui permettra d'atteindre les objectifs choisis.

\section{Du DPC à l'évaluation des compétences}

On peut aussi imaginer, qu'à moyen terme, des organisations professionnelles développent et valident des programmes d'évaluation des compétences basées sur la simulation permettant d'embrasser plusieurs champs tels que :

- la revalidation périodique des compétences des professionnels tout au long de leur carrière, à l'instar des pilotes de lignes ou comme cela a été proposé par le Mont Sinaï Hospital de New York pour la revalidation des compétences en anesthésie grâce à un véritable programme de réentraînement (3) ;

- le bilan des « savoir-faire » des nouveaux professionnels recrutés dans un établissement ;

- la remise à niveau des praticiens évoluant vers une nouvelle branche de la discipline (par exemple : anesthésie adulte vers anesthésie pédiatrique) ;

2. Loi HPST 2009. 
- le transfert de compétences d'activités médicales vers des activités paramédicales ;

- la validation de nouvelles compétences acquises tout au long du cursus ou la sélection des internes, comme cela se pratique dans certains centres étrangers (Royaume-Uni).

\section{Conclusion et propositions}

Compte tenu de tous ces éléments, la simulation couvre bien l'ensemble du champ du DPC. En effet, elle peut permettre aussi bien la formation procédurale à des gestes ou des situations techniques (formation classique d'apprentissage) que l'évaluation des pratiques professionnelles et des synergies d'équipe.

La simulation permet une véritable implication individuelle « vérifiable» ainsi qu'une amélioration significative de la performance individuelle et collective des professionnels confrontés à une situation de prise en charge.

Si une politique concernant la formation par la Simulation était définie, cela permettrait en particulier la mise à disposition de plates-formes mutualisées de formation par la simulation, autorisant ainsi l'accès à ce mode de formation au plus grand nombre de professionnels. L'enjeu majeur est de garantir une formation optimale des professionnels pour l'acquisition et le maintien de leurs compétences, afin d'améliorer la qualité et la sécurité des soins dans l'intérêt des patients.

En conclusion du rapport, dix actions sont proposées qui permettraient un développement structuré de la simulation et un impact incontestable sur la qualité et la sécurité des soins :

\section{Simulation en santé : 10 propositions}

\section{Proposition 1}

$\rightarrow$ La formation par les méthodes de simulation en santé doit être intégrée dans tous les programmes d'enseignement des professionnels de santé à toutes les étapes de leur cursus (initial et continu). Un objectif éthique devrait être prioritaire : " jamais la première fois sur le patient ».

\section{Proposition 2}

$\rightarrow$ L'importance de l'impact de la formation par la simulation sur les facteurs humains et le travail en équipe ainsi que son utilité dans la sécurité des soins doivent être largement étudiés.

\section{Proposition 3}

$\rightarrow$ Une politique nationale doit permettre à la formation par la simulation d'être valorisée et dotée de manière adaptée.

\section{Proposition 4}

$\rightarrow$ La formation initiale et continue par la simulation doit faire l'objet de coopérations entre les universités et les structures de soins ou les instituts de formation (publics ou privés).

\section{Proposition 5}

$\rightarrow$ Les formateurs en matière de simulation doivent bénéficier d'une compétence réelle, validée par l'obtention de diplômes universitaires spécifiques.

\section{Proposition 6}

$\rightarrow$ Chaque société savante doit identifier des programmes de formation par la simulation adaptés aux priorités de leur discipline.

\section{Proposition 7}

$\rightarrow$ L'ensemble des ressources doit faire l'objet d'une mutualisation selon des critères validés (plates-formes équipées accessibles, banque de scénarios, programmes de DPC, etc.).

\section{Proposition 8}

$\rightarrow$ Au niveau national ou régional, les accidents les plus graves ou les plus signifiants doivent faire l'objet de reconstitutions en simulation afin d'en analyser les causes et de prévenir leur répétition.

\section{Proposition 9}

$\rightarrow$ La simulation peut être utilisée comme un outil de validation des compétences (ou de transfert de compétences) des professionnels au sein de structures « certifiées ».

\section{Proposition 10}

$\rightarrow$ Les travaux de recherche sur la simulation en santé doivent faire l'objet d'une méthodologie rigoureuse et d'une collaboration en réseau. 



\section{Introduction}

Simulation et développement professionnel continu sont les deux concepts qui ont guidé la rédaction de la première partie de ce travail.

Dans un premier temps, il nous a paru important de définir la notion de « simulation » dans ce contexte puis, après un bref rappel historique, d'étudier l'apport de la simulation pour la formation " continue " dans certains métiers à risque. L'expérience de ces métiers nous est rapidement apparue indispensable à connaître en préalable à l'étude de la simulation en santé.

Celle-ci fait ensuite l'objet de l'état des pratiques internationales, tant à travers la littérature (enquêtes et revues) que par la vision de spécialistes reconnus. La visite de certains centres de référence a permis de préciser la réalité de ces pratiques.

La méthodologie et les résultats de deux enquêtes nationales, effectuées dans le cadre de la mission, sont rapportés. La première est une enquête " généraliste ", utilisant un questionnaire simplifié et destiné à appréhender une vision globale de l'utilisation des techniques de simulation au sein des établissements de santé et des écoles des métiers de la santé. La seconde, plus complète, a pour but de préciser l'organisation et le fonctionnement des centres de simulation installés ou en cours d'installation.

La dernière partie du rapport développe les aspects actuels de la formation par la simulation. Les techniques de simulation sont décrites, tandis qu'une revue de la littérature retient les points essentiels de la formation par cette méthode. Le lien est ainsi facilement établi avec le développement professionnel continu, au cours duquel la simulation peut représenter un outil particulièrement efficace.

Au terme de ce travail, des propositions générales sur la simulation en santé sont présentées.

\section{Définitions de la simulation}

D'après « Le Robert ${ }^{\circledR}$ », dictionnaire historique de la langue française, le verbe " simuler » est emprunté au $\mathrm{XIV}$ siècle au latin classique simulare avec les sens de :

- « représenter exactement », « copier », « imiter », « feindre », « prendre l'apparence de » ;

- « donner pour réel ce qui n'est pas, en imitant l'apparence de la chose à laquelle on veut faire croire ».

L'adjectif " simulé » semble plus usité que le verbe au moins jusqu'au XVIII siècle, et s'applique couramment à :

- « ce qui est feint »;

- « imité » (Rabelais).

Le mot « simulation » désigne une méthode de mesure et d'étude consistant à remplacer un phénomène, un système à étudier par un modèle plus simple, mais ayant un comportement analogue ("Petit Larousse $\left.^{\circledR} 》\right)$.

Plusieurs définitions de la simulation sont retrouvées dans la littérature ; il n'existe pas cependant a priori de définition consensuelle et internationalement reconnue. Nous en proposons quelques-unes : trois « généralistes » et une plus spécifiquement dédiée à la simulation en santé.

Si l'on recherche une définition encyclopédique de la simulation on retrouve celle-ci : "La simulation est un outil utilisé par le chercheur, l'ingénieur, le militaire, le médecin, etc. pour étudier les résultats d'une action sur un élément sans réaliser l'expérience sur l'élément réel. Le moyen le plus simple serait de tenter l'expérience, c'est-à-dire d'exercer l'action souhaitée sur l'élément en cause pour pouvoir observer ou mesurer le résultat. Dans de nombreux cas, l'expérience est irréalisable, trop chère ou contraire à l'éthique. 
On a alors recours à la simulation : rechercher un élément qui réagit de manière semblable à celui que l'on veut étudier et qui permettra de déduire les résultats $»$.

Pour certains auteurs [Pascal Béguin et Annie Weill Fassina (4)], la simulation est une méthode d'enseignement, de savoir-faire et d'habiletés utilisés dans des tâches pour lesquelles un enseignement direct s'avère impossible pour des raisons déontologiques (sécurité et sûreté), économique (coût du matériel) ou technique (très faible probabilité d'occurrence des incidents ou accidents). L'objectif est de permettre à l'opérateur d'apprendre à reproduire de la façon la plus réaliste et fidèle les comportements attendus.

Pour Leplat « La notion de simulation recouvre le plus souvent le cas où il est fait appel à un objet support, le simulateur, spécifié par un but lié très directement au travail ; mais il est d'autres catégories de simulation dans lesquelles le rôle de l'objet support n'est plus joué par un dispositif matériel mais par un être ou groupe humain ou par une situation symbolique ou virtuelle » (5).

En ce qui concerne la simulation en santé proprement dite, la définition suivante nous a paru intéressante, car elle intègre bien le rôle de la simulation dans la formation des professionnels de santé : "Le terme simulation en santé correspond à l'utilisation d'un matériel (comme un mannequin ou un simulateur procédural) de la réalité virtuelle ou d'un patient standardisé pour reproduire des situations ou des environnements de soin, dans le but d'enseigner des procédures diagnostiques et thérapeutiques et de répéter des processus, des concepts médicaux ou des prises de décision par un professionnel de santé ou une équipe de professionnels " (Chambre des représentants USA, 111 th congress 02-2009).

Les différentes approches de la simulation peuvent être résumées par le schéma ci-dessous [d'après G.Chiniara (6)]. Dans ce schéma, la zone grisée correspond aux méthodes de simulation les plus fréquemment utilisées aujourd'hui.

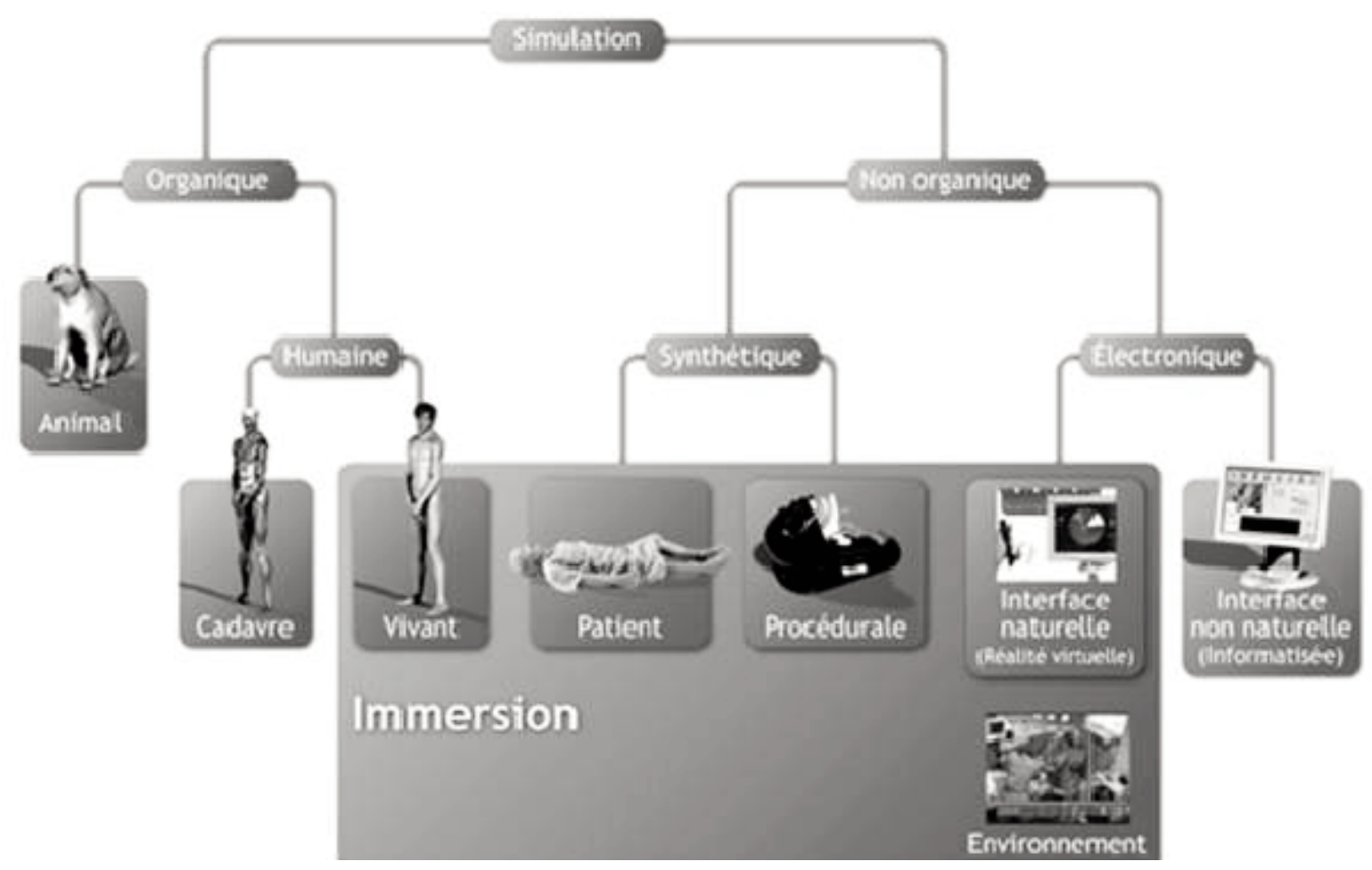

Schéma 1. Les différents champs de la simulation en santé 


\section{Historique de la simulation en santé}

(Contribution Dr Jérôme Berton, responsable du CESAR, centre de simulation du CHU d'Angers) $(7,8)$.

Au XVIII siècle, une sage-femme, Madame Du Coudray (9), décide d'enseigner aux matrones des campagnes «l'art des accouchements». Une partie de cet enseignement repose déjà sur l'utilisation d'une panoplie de mannequins qui permet de recréer des manœuvres obstétricales. Elle parcourt ainsi la France pendant vingt-cinq ans et forme plus de 5000 femmes grâce à la simulation. Au cours de sa campagne de formation, il est estimé qu'environ 4000 sages-femmes à travers la France ont utilisé ce mannequin, et que la mortalité infantile a montré ensuite une nette diminution.

De très nombreux exemples sont retrouvés dans l'histoire : Platon et Aristote évoquent déjà l'apprentissage par l'amusement et l'imitation de certaines activités, guerrières en particulier.

Au Moyen Âge, les chevaliers s'entraînent avec des simulateurs pour les joutes et les tournois permettant de développer leurs aptitudes au combat.

À la Renaissance, le jeu de rôle et le théâtre sont largement utilisés comme arts pédagogiques (10).

À partir de 1910 et jusqu'au milieu des années 70, un mannequin de bois, surnommé Madame Chases (du nom de sa conceptrice, fabricante de jouet) sera utilisé par les élèves infirmières, du Hartford Hospital Training School of Nurses, pour la pratique des soins de nursing de base. Ce modèle va se perfectionner et sera toujours utilisé par l'armée américaine durant la seconde guerre mondiale. Durant les années 50 , le Pr Peter Safar, du Baltimore City Hospital, tente de perfectionner les manœuvres de réanimation cardiorespiratoire. À cette époque, il doit encore mettre à contribution les membres de son équipe pour simuler les patients. Les volontaires sont alors endormis et intubés. Devant le manque évident de modèle de simulation, il va s'associer avec le médecin norvégien Bjorn Lind, pour tenter de développer un modèle adapté à la réanimation cardio-respiratoire. Le fabricant de jouet Asmund Laerdal, qui fabrique déjà des patients factices pour l'armée, développe avec les deux médecins le fameux mannequin Resusci Anne au début des années 60. À la même époque, les docteurs Stephen Abrahamson et Judson Denson mettent au point le premier mannequin contrôlé par ordinateur, le Sim One (11). II sera le modèle qui inspirera, par ses capacités et son réalisme, les mannequins haute-fidélité actuels (8).

À côté de l'évolution technologique, l'utilisation du patient standardisé (12) (un acteur simulant un patient) commence dès les années soixante aux États-Unis, initié par le Dr Howard Barrows (le premier " patient " simulera un cas de sclérose en plaques). Toujours à la même époque, un autre mannequin de simulation, Harvey, entièrement dédié à la cardiologie est mis au point par le Dr Michael Gordon. Ce mannequin peut mimer plus de trente pathologies cardiaques. Le développement de la programmation permet de mettre au point différents modèles physiologiques et pharmacologiques réalistes et adaptés à la pédagogie. Par exemple, le logiciel GasMan (développé par le Dr Philip en 1984) simule les échanges pharmacologiques de différents produits en anesthésie. En 1986, le Dr Gaba met au point le Comprehensive Anesthesia Simulation Environment (CASE), dans le cadre de ses recherches sur les facteurs humains et la gestion des crises en anesthésie. Ce simulateur était composé à son origine d'un simulateur de monitorage, d'une tête d'intubation modifiée et d'un bras de perfusion. Progressivement, le modèle va être perfectionné puis distribué sous le nom du Eagle Patient Simulator en 1995. À la même époque, un autre mannequin, tout aussi perfectionné, le Gainesville Anesthesia Simulator, peut analyser en direct les échanges gazeux. II sera encore perfectionné et commercialisé sous le nom de Human Patient Simulator par le distributeur METI. Un modèle moins perfectionné, mais adapté à la médecine d'urgence, le SimMan, est proposé en 2000 par Laerdal. Aux États-Unis, en 2001, la publication du rapport « to Err is Human » (13) permet une prise de conscience de l'importance du facteur humain dans les erreurs médicales, et propose de positionner la simulation médicale comme l'un des moyens d'en réduire la fréquence ou les conséquences. 


\section{Simulation en dehors de la santé}

Dans le cadre de notre mission, nous avons souhaité découvrir l'expérience d'autres métiers. II nous a paru important de rapporter leur témoignage au début de ce rapport, en raison de la similitude des pratiques observées. Nous avons choisi trois « métiers » à risque : l'aéronautique, la marine marchande et l'industrie nucléaire.

\subsection{Simulation en aéronautique}

Deux contributions sont ici rapportées : les enjeux de la simulation en aéronautique (René Amalberti, docteur en médecine et en psychologie, chargé de mission auprès de la direction générale de l'aviation civile depuis 1994) et la formation des aiguilleurs du ciel (Florence-Marie Jegoux, responsable de la formation des aiguilleurs du ciel, subdivision instruction, subdivision Nantes Atlantique Ouest).

\section{Enjeux de la simulation en aéronautique}

L'aéronautique est considérée comme le berceau de la simulation professionnelle à grande échelle.

Les premiers simulateurs de vol (on devrait dire de machines volantes) sont apparus en début du $\mathrm{XX}^{\mathrm{e}}$ siècle, concomitamment aux vrais avions ; ils ont mélangé très tôt des capacités de découverte des commandes et des procédures, d'émulation du vol, et un usage tourné vers la sélection pour tester les aptitudes spatiales des candidats.

II faut dire que la sanction répétée des accidents mortels lors de l'entraînement initial sur des machines inconnues, particulièrement des chasseurs monoplaces, a très vite motivé l'adoption d'une découverte première de l'appareil sur simulateur, d'abord limitée techniquement à l'environnement de base du cockpit et à l'apprentissage des procédures, puis de plus en plus précise et réaliste, restituant le vol et le mouvement.

Ces acquis militaires ont diffusé naturellement à l'aviation civile dès l'après guerre.

On notera toutefois que la formation en vol réel avec instructeurs perdure, y compris de nos jours pour les acquisitions ab initio (la formation initiale de pilote privé et de pilote de ligne), et ce même si cette formation initiale est de plus en plus aidée par des simulateurs simplifiés, voire partiels, qui servent à enseigner la navigation, le maniement de secours de certains équipements, etc. L'arrivée massive de micromondes réalistes avec des simulateurs de vol sur PC à très bas coûts n'y change rien. En bref, on ne peut pas apprendre à voler sans voler. Le nombre de vols réels pour chaque niveau de qualification de pilote (pilote privé, pilote professionnel) est même imposé, non dérogeable et fixé par les règles internationales.

Le simulateur va, en revanche, être utilisé massivement pour les formations de reconversion spécifique à chaque appareil qui rythment la carrière des pilotes, et pour leurs séances de réentraînement continu imposées par les règlements tous les six mois.

C'est évidemment un outil rêvé pour ce faire, capable d'entraîner les pilotes à des pannes rares ou des pannes qui sont trop dangereuses pour être produites en vol réel.

La première qualification sur un appareil (on parle de Qualification de Type : QT) autorisée sans aucun vol dans le cursus par les autorités internationales de l'aviation civile remonte à la fin des années quatre-vingts chez Airbus (qualification sur Airbus A310) : les pilotes venaient pour un stage de cinq semaines, découvraient pendant quinze jours l'appareil et ses fonctions de façon interactive sur un système d'apprentissage par ordinateur, puis entraient dans une série de simulations sur simulateur à base fixe qui leur faisait découvrir l'appareil, les procédures et la gestion des pannes les plus habituelles. La dernière semaine était consacrée à des séances sur un simulateur mobile, où l'équipage en formation devait affronter des conditions plus dégradées. Aujourd'hui, les stages se sont raccourcis, mais plus aucune QT chez aucun constructeur mondial n'utilise de vol réel (trop cher, trop dangereux). 
En résumé, les règlements internationaux de l'aviation civile imposent aujourd'hui la simulation comme outil de formation continue et d'entraînement obligatoire aux procédures et pannes. Les pilotes sont évalués et qualifiés sur cet outil. Des inspections en vol viennent ponctuellement compléter le dispositif.

\section{$\rightarrow$ Personnes concernées par la formation}

On l'aura compris, l'ensemble des pilotes professionnels est soumis à la formation continue sur simulateur depuis plus de vingt ans.

Mais la formation continue sur simulateurs concerne bien plus de personnels ; elle s'est imposée dans les années quatre-vingts aux personnels de cabine pour l'évacuation (toboggans, ouverture des portes et issues de secours, etc.) et aux contrôleurs aériens pour leur entraînement initial et continu.

Elle est en revanche à peine débutante en formation continue pour la maintenance (enseignement assisté par ordinateur plus que simulation). Dans ce domaine, elle existe surtout en Conception Assistée par Ordinateur (CAO) grâce au logiciel CATIA (Dassault System), qui se présente comme un outil exceptionnel permettant de faire vivre le produit avant qu'il n'existe (intérêt technique et commercial), de le visiter, de le régler, et de tester à l'avance toutes les opérations de maintenance, leur faisabilité, les habilités requises et comment préparer leur enseignement (mannequins électroniques simulant les mécaniciens interagissant avec l'environnement émulé par l'ordinateur et testant l'anthropométrie requise pour réaliser chaque réparation : quelle taille, quelle force, quelle corpulence, quel savoir-faire, quel outil, avec en retour des modifications de conception chaque fois que la maintenance va s'avérer trop difficile).

Dans tous les cas, l'aéronautique est certainement le secteur industriel mondialisé où il y a le plus de simulateurs de tous ordres, le plus de règlements les imposant ${ }^{3}$ dans la formation continue, et le plus grand taux de satisfaction des professionnels.

\section{$\rightarrow$ Types de simulation}

L'aéronautique utilise toute la gamme de simulations possibles:

- jeux électroniques (micromondes réalistes), aujourd'hui extrêmement réalistes et accessibles sur ordinateur. Ces jeux de simulation ne sont pas reconnus par les autorités, relèvent d'achat personnel, n'ont pas de place obligatoire dans les cursus, mais ils sont encouragés et participent au maintien général des compétences aussi bien en aviation militaire que civile ;

- simulateurs de conception, domaine particulier presque entièrement résumé à CATIA, le logiciel de CAO tellement puissant qu'il s'est imposé comme un standard mondial. Ce simulateur informatique est certifié ;

- simulateurs informatiques d'apprentissage : ce sont des simulateurs informatiques plus sophistiqués que les jeux sur ordinateur, souvent vendus avec la machine comme outils d'entraînement libres et bas coûts, parfois intégrés au cursus de formation. Ces simulateurs n'ont pas d'obligation légale ;

- simulateurs simplifiés : il s'agit de cockpits ou d'entraîneurs au contrôle aérien sur une base en dur, assez réaliste mais qui n'émule qu'une partie du travail : découverte de l'interface de dialogue de la machine, travail sur la navigation, etc. Ils sont achetés par les institutions, souvent posés en self-service dans les centres opérationnels militaires, de contrôle aérien, de secteurs de compagnies aériennes. Ils ont parfois des obligations réglementaires, et sont dans ce cas certifiés par les autorités de l'aviation civile (simulateur de toboggans d'évacuation par exemple);

- simulateurs réalistes complets : il s'agit de simulateurs d'entraînement surtout réservés à la formation continue, hautement réalistes pour l'interface et pour le modèle de processus à contrôler (le vol, le contrôle aérien). Ces simulateurs sont certifiés et ont des obligations réglementaires à la fois dans leur performance, leur usage et leur contrôle ;

3. Arrêté du 27 juillet 2006 modifiant différents arrêtés relatifs aux membres d'équipage de conduite d'avion. 
- sans oublier les jeux de rôles dans la gestion des conflits de l'équipage, qui ont été largement utilisés dans les formations " facteurs humains », elles aussi obligatoires sous l'acronyme CRM (Crew-Resource Management).

\section{$\rightarrow$ Moyens alloués}

Les moyens alloués à la simulation en aéronautique sont considérables. On ne parlera pas ici de formation initiale. Mais la seule formation continue en aéronautique constitue un poids moyen de 10 à $15 \%$ du budget total d'une compagnie ou d'un système de contrôle. L'imposition réglementaire est si forte (pas de maintien de qualification sans formation continue) que l'aéronautique a intégré le temps de formation dans le provisionnement de ses effectifs, et considère ainsi qu'un secteur normal de travail doit avoir $110 \%$ de postes par rapport à l'usage imposé par le service proprement dit. Les $10 \%$ supplémentaires sont réservés au temps de la formation ou à des décharges partielles d'activités au profit d'activités de cadres et d'instruction.

\section{$\rightarrow$ Difficultés et limites}

L'aéronautique a tout misé sur la formation aux procédures normales et anormales (entraînement à des pannes rares ou des situations difficiles). Le simulateur est un outil parfait pour cela.

En revanche, l'usage de l'outil pour des situations plus complexes, plus liées à des comportements humains a toujours posé problème.

À la fin des années 80 puis au début des années 90, l'OACI (Organisation de l'aviation civile internationale) a préconisé des entraînements facteurs humains pour gérer les conflits à bord, les problèmes de communication, les problèmes de distractions, etc. L'interprétation de ces demandes a conduit à mettre au point deux types de formations :

- les CRM (Crew-Resource Management) : des cours résidentiels pour quinze à vingt participants d'une durée de un à deux jours sur la façon de se comporter avec des jeux de rôles et des saynètes montrant des professionnels en difficulté pour lesquels réagissent les participants ;

- les LOFT (Line Oriented Flight Training) sont des séances de simulation annuelles imposées, où le scénario est plus complexe, avec une interactivité plus importante concernant le contrôle aérien et les incidents non nécessairement techniques par rapport aux entraînements liés aux procédures.

Le résultat est discutable et discuté sur le long terme. Les CRM et les LOFT sont pratiqués parce qu'ils sont obligatoires et réglementaires, mais leur contenu a souvent été pointé du doigt comme se dégradant au fil du temps pour devenir des scénarios trop simples, vite connus de tous et peu porteurs d'apprentissage réel. Le système a particulièrement souffert dans la durée, de l'érosion des formateurs motivés sur ces sujets : les premiers instructeurs l'étaient, les suivants ont plus été contraints et sont sans doute moins psychologiquement préparés et entraînés à des debriefings complexes. Un effort international est en cours pour corriger ce problème, mais il se heurte à d'autres priorités.

Jusqu'à présent, et au nom d'une confiance qu'il faut préserver à tout prix dans le système et les procédures, les autorités et l'industrie aéronautique se sont totalement refusées à injecter dans les simulations facteurs humains des pannes, conflits ou situations très rares qui conduiraient à mettre en doute la machine et le contrôle aérien, ou pire à former des opérateurs à prendre des initiatives et récupérer une autonomie que personne ne souhaite qu'ils reprennent.

Inversement, l'aéronautique a mis l'accent, à partir de 1998, sur la nécessité d'évaluer ces formations facteurs humains devenues obligatoires, mais faites au départ sur des bases volontaristes. L'évaluation est aujourd'hui imposée mais ne fonctionne pas très bien, et se limite finalement à une évaluation de conformité aux procédures. Plus globalement, l'utilisation de milliers d'instructeurs et d'évaluations professionnelles directes et sanctionnantes a toujours pour corollaire un encadrement fort, afin de ne pas créer de jugement trop personnel vis-à-vis du regard soupçonneux et scrupuleux des syndicats. C'est un point sur lequel l'aéronautique a très bien réussi concernant la formation aux procédures, moins bien pour filtrer par la simulation ses professionnels les plus marginaux ou dangereux. 


\section{$\rightarrow$ Résultats (en termes de satisfaction et si possible d'efficacité, etc.)}

Personne n'imaginerait revenir sur le passé en aéronautique. La simulation est l'outil de la formation continue. Les économies en prix sont sans précédent (un vol à vide coûte $50000 €$ à $100000 €$ selon la machine), et la formation évidemment de grande qualité, puisqu'elle peut entraîner les personnels à ce qu'ils voient tous les jours et à ce qu'ils n'observeront jamais.

En termes de prospective, la simulation en aéronautique n'est pas dans une phase de révolution ; on peut parler aujourd'hui de simple continuité.

\section{Aiguilleurs du ciel}

Au fur et à mesure de l'évolution des technologies dans le contrôle aérien, la simulation s'est imposée comme un moyen essentiel pour former les contrôleurs. Toutefois, tous les pays n'utilisent pas la simulation de la même manière, souvent pour des raisons économiques.

\section{$\rightarrow$ Avantages et inconvénients}

Les avantages de cette méthode de formation sont variés. Le fait d'être détaché du trafic réel a des avantages pédagogiques et sécuritaires. Les instructeurs peuvent aller plus loin dans les situations ; ils peuvent tester les limites des élèves en toute sécurité et leur faire comprendre par eux-mêmes certaines erreurs de raisonnement; ils peuvent programmer une séance en fonction du niveau de l'élève, ou en ciblant une difficulté particulière, ce qui est impossible dans la réalité, où l'on subit la quantité et la complexité du trafic par nature imprévisibles.

Le simulateur permet également de maintenir certaines aptitudes et capacités professionnelles, en particulier quand le niveau de trafic baisse pour des raisons économiques ou saisonnières.

En revanche, il est difficile de concevoir et d'effectuer des simulations qui correspondent complètement à la réalité, avec la part de surprise et d'imprévu.

\section{$\rightarrow$ Formation initiale}

Actuellement, dans l'aviation civile, le simulateur est utilisé en formation initiale, à l'ENAC de Toulouse (École nationale de l'aviation civile), avec des simulateurs correspondant aux différents métiers et différentes phases de formation des contrôleurs : contrôle tour, contrôle d'approche, contrôle en route. Les examens de contrôle aérien se déroulent donc obligatoirement sur simulateur pendant les études.

La dernière partie de la formation initiale des contrôleurs se déroule dans les centres d'affectation. Et comme pour la formation continue, les moyens de simulation dépendent du centre : plus le centre est important, plus la formation sur simulateur est importante. Par exemple à Nantes, les élèves doivent passer quatre journées réglementaires sur simulateur, dont un stage de qualification (examen) nécessaire à l'obtention de la qualification du centre ; auxquelles se rajoutent une journée de formation initiale à l'approche et des journées de formation en fonction de la demande de l'élève ou des instructeurs.

\section{$\rightarrow$ Formation continue}

Pour la formation continue, il est obligatoire d'effectuer un stage "Situations inhabituelles et dégradées » tous les trois ans. Ce stage comporte une demi-journée de théorie et un jour et demi de simulateur. Le programme permet de rester à jour sur toutes les situations rares et critiques : pannes de tous matériels côté contrôleur, phénomènes météo particuliers, problèmes avions divers (pannes, déroutements, détournements, alertes, détresse, malades à bord, etc.), fermeture de piste, accidents, etc.

Pour les contrôleurs qui ne font que du contrôle « tour ", il n'existe pas encore de simulation « tour » dans les services (hormis à l'ENAC). Ce stage se déroule donc uniquement de manière théorique, et les contrôleurs ayant expérimenté les deux systèmes de formation sont unanimes sur la prédominance de la simulation sur la théorie. 


\section{$\rightarrow$ Réglementation}

Ce temps passé au simulateur est différent dans chaque centre, car le centre élabore lui-même son programme de formation qui est ensuite validé par les autorités de surveillance (Programme de formation d'unité pour la formation initiale, et Programme de compétences d'unité pour la formation continue).

Pour les constructions de nouveaux aéroports, pour tous les changements importants de la structure des espaces aériens, il est obligatoire de réaliser les formations des contrôleurs au moyen de simulateurs, puisque les structures n'existent pas encore, que les temps de formation sont longs, que la formation doit être terminée et les personnels compétents le jour $\mathrm{J}$.

\section{$\rightarrow$ Résultats}

Le bénéfice de la simulation est très difficilement quantifiable et séparable du reste de la formation. La simulation est actuellement considérée comme un passage obligatoire, que personne ne veut ni ne peut remettre en cause. La tendance serait de manière très nette pour l'allongement des temps de formation sur simulateur.

\subsection{Simulation en marine marchande}

(Contribution rédigée par J.P. Clostermann, capitaine illimité, professeur de l'enseignement maritime et instructeur sur simulateur à l'ENSM du Havre)

Introduite dès le début des années 80 pour l'entraînement au travail sur radar, la simulation a depuis étendu son champ d'activité à tous les domaines du navire et augmenté sa fidélité jusqu'au niveau full mission, niveau dans lequel le réalisme des situations permet de travailler - au-delà des tâches techniques - les comportements (14).

\section{- Enjeux}

L’enjeu majeur de la simulation est un enjeu de société : la sécurité ; l'accident n'est plus tolérable.

Cet enjeu explicite global mobilise de multiples enjeux intermédiaires :

- pédagogiques : le travail en dynamique permet d'introduire l'entropie qui rend la validité d'une décision limitée dans le temps et fait prendre conscience de la notion d'irréversibilité ;

- politiques : il était difficile de rester à l'écart d'une tendance lourde. De plus la compétition ouverte dans une activité mondialisée impose à l'enseignement maritime d'innover sans cesse pour rester dans le peloton de tête et justifier la présence de marins français à bord ;

- financiers : les assureurs, payant en dernier ressort, poussent à l'utilisation de la simulation afin d'augmenter la compétence pour diminuer l'accidentologie ;

- techniques : dans la conception d'un projet maritime, la simulation permet de valider des options, comme la taille maximale des navires ou de fixer a priori des limites pour l'exploitation d'un terminal.

- judiciaires : cet enjeu est apparu ces dernières années avec l'intensification des recherches en responsabilité après des événements de mer : les stages de formation continue sur simulateur permettent aux compagnies de navigation de voir s'éloigner un reproche de négligence.

- réglementaire : (Annexe 1. Arrêté du 30 juin 1999 relatif à l'utilisation des simulateurs dans les formations conduisant à la délivrance de titres de formation professionnelle maritime). L'OMI (Organisation Maritime Internationale), qui tente depuis soixante ans de réglementer une activité historiquement très libre, impose de plus en plus le recours à la simulation pour concilier les exigences contradictoires du commerce maritime mondial : davantage d'officiers, plus vite et mieux formés et si possible déjà en possession de savoir-faire. 


\section{- Public : étudiants et navigants confirmés}

En formation initiale, la simulation permet de valider une compétence en situation, là où auparavant il n'était possible de valider que des connaissances ; en formation continue, elle met à jour les savoir-faire dans un environnement technologique en évolution rapide et les réactive lorsque le métier n'a pas permis, temporairement, de les exercer. Comme dans l'aéronautique, brevets et certificats ont une durée limitée et sont soumis à des revalidations périodiques, sauf s'il est prouvé que les savoir-faire précisément concernés n'ont pas cessé d'être exercés.

\section{Moyens alloués}

À l'heure actuelle, il n'y a pas de moyens spécifiques alloués à la simulation en elle-même. Les établissements d'enseignement, du niveau académique ou de niveau intermédiaire choisissent la simulation parmi d'autres investissements possibles. La montée en puissance de ces outils très coûteux en acquisition, entretien et frais de fonctionnement (un à deux instructeurs pour huit stagiaires), explique peut-être pour une part les façades parfois délabrées de certaines écoles maritimes. Des partenariats public-privé commencent à se mettre en place.

\section{- Types de simulation}

L'outil, qui simule une partie de l'installation, permet d'apprendre les gestes techniques : simulateur radar, installation radio ou carte électronique, ou de valider un module de connaissances par les savoir-faire : simulateur de chargement par exemple.

Le simulateur dit full mission permet par le réalisme de l'installation d'exercer l'ensemble des savoir-faire, mais surtout de travailler sur les comportements en conduite du navire.

Un autre type de simulation est dit « de bas niveau » : une situation schématique est projetée sur un écran. Elle stimule la réflexion et permet de travailler efficacement en petits groupes sur les processus de prise de décision : ici se vérifie particulièrement l'adage : la simulation n'est qu'un prétexte au debriefing.

\section{Bénéfices}

Ils sont difficiles à quantifier, d'autant plus que les lieux de l'activité étant très éloignés de ceux de l'enseignement, les retours des établissements sur la qualité de leur formation sont très limités.

Le signe le plus tangible de retour positif est que les compagnies de navigation n'hésitent plus aujourd'hui à investir des sommes importantes, afin de posséder leurs propres installations de simulation, ce qui pose la question de la qualité de la pédagogie mise en œuvre.

\section{Conclusion pour la simulation dans le maritime}

Magnifique outil d'acquisition de la compétence professionnelle, la simulation mérite que toute la pédagogie soit repensée, afin que les connaissances théoriques ne soient plus le cœur de l'enseignement, mais prérequis à des mises en situations qui valideront la présence des savoir-faire indispensables à l'exercice du métier. Un bémol cependant : la simulation ne peut remplacer l'expérience pour la détection des signaux faibles, ceux qui, grâce à des mois ou même des années de métier, permettent à l'expert de déceler, au milieu d'un bruit de fond confus, l'anomalie significative. La simulation ne transforme pas un novice en expert, elle aide le novice à faire ses premiers pas de professionnel dans des conditions de sécurité améliorées. 


\subsection{Simulation dans l'industrie nucléaire}

(Contribution rédigée par M. Philipe Fauquet-Alekhine, consultant facteurs humains au CNPE de Chinon et chercheur au laboratoire de recherche, pour les sciences de l'énergie)

\section{Contexte : installation et pilotes}

L'exploitation des centrales nucléaires nécessite un haut degré de maîtrise des installations, que ce soit en termes de pilotage ou de maintenance ou en situation normale ou accidentelle. II en va de la sécurité et de la santé des populations, et donc de la possibilité de maintenir la filière nucléaire sur le marché de l'énergie. L'exploitant nucléaire doit ainsi être capable non seulement de maintenir son savoir-faire, mais également de l'actualiser afin de s'adapter aux nouveaux impératifs pouvant intervenir au niveau de la sûreté ou de la sécurité, de la réglementation ou de la législation (15) ou de l'économie.

Plusieurs champs sont exploités en vue de garantir ces impératifs : l'aspect technique en premier lieu puisqu'il s'agit de faire fonctionner un système technique. Cette dimension reçoit toute l'attention des ingénieurs, de la conception au démantèlement d'une centrale, en passant par la construction et l'exploitation. L'aspect organisationnel ensuite est fondamental, avec une volonté permanente d'analyse et d'adaptation de cette organisation, en prenant en compte l'homme dans toute sa dimension, puisqu'il s'agit d'exploiter un système sociotechnique $(16,17)$.

De nombreux autres champs sont exploités en permanence, parmi lesquels ceux mis en œuvre pour répondre à la capacité de développer et de maintenir le savoir-faire $(18,19)$ : EDF dispose pour cela de simulateurs de pilotage de réacteur nucléaire. Les salles de commande sont reproduites à l'échelle 1 (simulateurs " pleine échelle »), et des calculateurs permettent en temps réel de simuler les paramètres physiques de l'installation. Le choix d'un tel outil pédagogique est motivé notamment par une double nécessité : créer des situations les plus proches de la réalité d'exploitation pour entraîner une équipe à piloter un système technique complexe en collectif. Dans cette perspective, le simulateur « pleine échelle » a démontré toute sa plus-value pour l'industrie nucléaire.

Les acteurs de la situation simulée sont les membres de l'équipe de conduite ${ }^{4}$ et les instructeurs ou formateurs. Une équipe de conduite de réacteur nucléaire est généralement composée d'une quinzaine de personnes qui ont en charge le pilotage et l'exploitation d'une paire de réacteurs et équipements associés. La mission de l'équipe de conduite est de piloter le réacteur en fonction de la demande pour produire de l'électricité à partir de l'énergie nucléaire, tout en garantissant la sûreté des installations. Au sein de cette équipe, quatre à six personnes sont concernées directement par le pilotage, les autres étant rattachées aux manipulations d'organes sur l'installation directement.

\section{Conception technique et pédagogique : genèse et développement}

L'entreprise EDF (Électricité de France) s'est dotée de différents types de simulateurs pour le pilotage : des simulateurs partiels et des simulateurs pleine échelle.

Les simulateurs partiels représentent des parties de la salle de commande, et focalisent sur un ensemble élémentaire de l'installation que les pilotes doivent apprendre à connaître, avant d'embrasser le fonctionnement.

Les simulateurs «pleine échelle » sont constitués de la salle de commande dans son ensemble, réplique identique de la réalité d'exploitation, d'un calculateur, et d'un pupitre de contrôle d'où les instructeurs gèrent la situation de simulation. Ce pupitre est fermé par des vitres sans teint et équipé d'un système vidéo numérique, relié à plusieurs caméras permettant des points de vue divers de la salle de commande. L'ensemble des vues de caméras est enregistrable et exploitable par la suite en salle de debriefing. Ce système vidéo présente une plus-value indéniable, et est soumis à une déontologie stricte.

L'utilisation du simulateur est cadrée par des scénarios qui permettent d'initier la situation de travail et de

4. L'équipe de conduite désigne l'équipe en charge du pilotage du réacteur. 
proposer un enchaînement technique du process pendant la situation simulée. L'ensemble est coordonné par les instructeurs.

Les instructeurs ont des profils de carrière divers. Être issu du métier donne une certaine légitimité d'emblée face aux personnes en formation, et permet d'agrémenter la formation d'exemples concrets ; être novice favorise la prise de recul par rapport à la situation simulée et évite à l'instructeur de s'engager dans des débats exclusivement techniques.

L'ensemble de ce qui est mis en œuvre en situations simulées et en debriefing est essentiellement issu des travaux de recherche réalisés par Pastré, Samurçay, et Plénacoste, de 1996 à 2001 (20,21). Ils donnent une description sous l'angle conceptuel des situations de simulation et de debriefing (22), travaux complétés par Fauquet (23).

Pour résumer, retenons que différentes modalités de travail existent sur simulateur en fonction des objectifs recherchés : professionnalisation initiale, recyclage, application de procédures accidentelles, développement des savoir-faire. La professionnalisation initiale est elle-même décomposée en différentes phases : découverte et appropriation du simulateur, fonctionnement basique de l'installation, fonctionnement en situation de pannes. Cette approche progressive est fondamentale, car elle permet de placer les apprenants dans de bonnes conditions d'apprentissage : des travaux récents ont montré qu'une mise en situation de simulation de crise sans approche progressive plaçait la plupart des apprenants dans une zone de désordre cognitif néfaste à l'apprentissage (24).

Pour atteindre ces objectifs, des méthodes d'analyse de pratiques sont utilisées (cf. ci-dessous). Ce dispositif permet de réinterroger ce qui est réalisé par l'équipe comme ce qui ne l'est pas, les moteurs ou les freins, avec une perspective de transformation éventuelle, individuelle ou collective, ou une prise de conscience concourant à l'ancrage de ces pratiques professionnelles et éventuellement permettant de les penser autrement (25). La prise de conscience, d'abord individuelle, diffuse dans le collectif pour s'intégrer dans un genre professionnel (26).

L'effet recherché, lors du debriefing de séance, passe par la distanciation des pilotes concernant leur action lors de la situation simulée qu'ils viennent de vivre.

Le debriefing renvoie aux méthodes d'analyse de l'activité de travail développée par Yves Clot (26-30) et s'apparente, par certains de ses aspects, aux Crew-Resource Management pratiqués par les compagnies aériennes.

Le passage en situation de simulation d'une équipe de conduite est réalisé en moyenne sur trois jours, divisés la plupart du temps en une séance de 3 heures (passage sur simulateur) et un debriefing de 3 heures, séparés par une pause de trente minutes.

II faut garder à l'esprit qu'une situation de simulation n'a de sens qu'en associant des durées de debriefing adaptées, donnant le temps de discussion nécessaire. En d'autres termes, croire qu'une bonne situation de simulation est celle qui privilégie le temps de passage sur simulateur est une erreur.

\section{Extension : études, passage à l'évaluation et entraînement maintenance}

Au-delà de la formation initiale, du développement ou de l'apprentissage de nouvelles méthodes (31), le simulateur est un lieu privilégié d'études (30,32-34).

Formation, étude, etc. et évidemment évaluation ! Depuis plus de dix ans, les capacités des personnels formés au pilotage des réacteurs sont soumises à évaluation initiale, mais le renouvellement de la validation des capacités n'est en œuvre que depuis 2005. Sur ce point, il faut noter la difficulté rencontrée par la compagnie afin de mettre en place ce système d'évaluation continue : la décision de direction s'est vue confrontée, dès le début du projet, à une opposition forte d'une certaine catégorie de personnels fortement relayée par les organisations syndicales. L'un des enseignements de cette situation est que, pour éviter ce 
genre de conflit, l'évaluation continue par la simulation doit être intégrée très tôt dans l'organisation, sinon les habitudes prises peuvent délégitimer le simulateur comme outil d'évaluation.

Les progrès, induits par la formation sur simulateur, ont été tels que la direction de production nucléaire d'EDF a récemment pris deux décisions majeures, lourdes de conséquences organisationnelles et financières, mais fructueuses en termes de développement de compétences et de résultats sûreté. À la fin des années quatre-vingt-dix, chacun des vingt sites nucléaires français a été doté d'un simulateur de pilotage. Puis en 2006, la direction a choisi d'étendre cette action pédagogique à d'autres métiers que le pilotage : chaque site nucléaire a été doté d'un simulateur de maintenance "Pleine échelle « dans des structures dites « chantier école » (200 m2 reproduisant un environnement industriel dédié à la formation par la simulation).

Quantifier le résultat, induit par de telles mesures, est difficile car elles font toujours partie d'un plan d'action ; ce qui peut être mesuré est le résultat de l'ensemble de ces actions conjointes. Pour ne donner qu'un seul chiffre, depuis 2006, le nombre d'arrêt automatique réacteur du parc nucléaire français a été réduit de plus de $20 \%$, ce qui est considérable.

\section{- Avantages, inconvénients, limites}

Parmi les bénéfices d'une formation sur plusieurs jours consécutifs, nous pouvons pointer : l'existence d'un temps d'intégration d'une séance à l'autre, la mémorisation récente des évènements vécus sur simulateur d'un jour à l'autre, et la facilité d'ajustement de la progression pédagogique.

Le simulateur permet également de varier la cinétique des phénomènes physiques dans deux sens : gel du simulateur pour comprendre ce qui se passe, ou accélération d'un phénomène lent pour éviter l'attente. Toutefois, en parallèle de l'avantage pédagogique de compréhension, les apprenants courent le risque de ne pas s'entraîner à la gestion de la cinétique réelle.

Pour aider les personnes en formation, les instructeurs suivent leur évolution d'une formation à une autre par un système de FAP, Fiche d'aide à la progression ou à l'aide d'un contrat de transfert collectif rédigé à la fin des trois jours.

La séparation physique instructeurs/équipe de conduite présente plusieurs avantages : elle favorise une mise en situation des acteurs, permet une concertation entre instructeurs sans perturbation ni interférence avec les acteurs de l'équipe, et favorise la prise de notes.

L'une des contreparties d'un instructeur, issu d'un métier de conduite ou de maintenance, est qu'il peut s'engager dans des débats techniques pendant le debriefing. La difficulté réside à garder la distance nécessaire pour ne pas tomber dans un tel piège. Cependant, la solution ne peut pas être opposée, soit choisir des instructeurs qui n'ont pas une réelle expérience professionnelle, car cela impose d'accéder à une légitimité, a priori plus acquise. Il est nécessaire pour l'instructeur, non issu des métiers de la conduite, de connaître a minima ce qui fait la technicité de ces métiers. Cependant, il pourra gagner une légitimité par l'utilisation de techniques ou de méthodes appropriées afin d'aider, par son questionnement, les acteurs à analyser leurs pratiques. Le fait qu'il ne soit pas du métier lui ouvre alors des portes pour un questionnement productif des acteurs.

Lorsque utilisé pour la formation par des professionnels de la formation, le simulateur se révèle être un outil remarquable. S'appuyer sur une telle compétence est fondamental, s'en priver peut devenir dangereux : les risques premiers peuvent être la déconstruction de savoir-faire, de collectif, voire l'implantation de mauvaises pratiques. Car même sans être incompétents, les formateurs peuvent générer des résultats leur échappant dans un premier temps pour leur revenir via l'analyse des événements indésirables : de nouveau intervient le problème de l'effet produit par l'entraînement sur simulateur. En chirurgie par exemple (35), une simulation virtuelle où le patient virtuel peut « mourir » sans conséquences, avec possibilité de rejouer la situation jusqu'au succès de l'opération, pose une question majeure : entraîne-t-on à opérer ou à banaliser la mort ou les deux? 


\section{- Perspectives (simulations hybrides, virtuelles, Serious Games)}

Ainsi, proposer aux équipes de conduite de travailler en situation de simulation contribue à fiabiliser les activités de travail, dans la mesure où les pratiques sont réinterrogées, repensées pour une nouvelle élaboration individuelle et collective. Ce point est fondamental pour la gestion des systèmes industriels à risque, quand les recherches montrent que cette gestion a généralement tendance à migrer vers des zones de fonctionnement moins sûres que ce que prévoyait le concepteur à l'origine (36). La mise en situation sur simulateur permet de réinterroger la pertinence des modalités d'actions mises en œuvre par les acteurs dans un tel système sociotechnique.

Toutefois, l'exploitant se heurte à un problème d'investissement, car les objets techniques pilotés par calculateur que sont les simulateurs coûtent très chers à l'achat et à l'entretien. Les évolutions technologiques sont néanmoins là pour réduire ce coût et ouvrir de nouvelles perspectives. Certains développeurs ont conçu des systèmes hybrides qui allient l'environnement de travail des pilotes (les pupitres de commandes taille réelle) et l'imagerie virtuelle (le pupitre ne comporte aucun bouton réel, mais est lui-même un grand écran LCD tactile qui reproduit les boutons et indicateurs configurables par simple touché de l'écran). En parallèle se développe actuellement le marché des Serious Games qui plonge l'apprenant dans un monde totalement virtuel représentant au plus près la réalité d'exploitation. La réduction des coûts est considérable puisque, du simulateur, il ne reste que le calculateur, l'objet technique devenant obsolète. La question qui doit alors être posée porte sur ce que l'on perd avec de tels systèmes du point de vue de l'intégration des savoir-faire, car la pratique professionnelle ne s'incorpore plus de la même façon (37). Ce champ reste à explorer.

\subsection{Synthèse de ces contributions}

L'ensemble de ces contributions présente indéniablement des points communs.

La mise en place de la formation par la simulation dans les industries à risques que l'on considère aujourd'hui comme activités ultra sûres a répondu à deux motivations :

- la première est la conviction profonde que la simulation pouvait apporter une amélioration de la sécurité. Ce concept, n'ayant n'a pu être aujourd'hui démontré, reste cependant pour ces secteurs une évidence sur laquelle on ne peut revenir. Le deuxième élément, qui a motivé la mise en place, est l'enjeu économique. En effet, il est moins coûteux d'entraîner des pilotes sur des matériels à terre que de faire voler un avion de ligne à vide. Dans l'industrie nucléaire, des gains financiers ont même été mesurés par une diminution de $20 \%$ de la fréquence des arrêts automatiques des réacteurs français.

- d'autres points communs méritent d'être retenus. En premier lieu, la formation par la simulation a d'abord été utilisée en formation initiale ou continue avec des moyens technologiques (simulateurs, mannequins, etc.). Elle s'est ensuite rapidement intéressée aux comportements des professionnels puis à la validation de leurs compétences. Les facteurs humains, liés aux activités en équipes, sont entrés progressivement dans le champ de la simulation, car il a été démontré dans ces secteurs qu'ils sont un des éléments prépondérants de la sécurité. Enfin, il est clair que dans tous les cas des investissements très importants ont été réalisés pour la conception et l'achat de simulateurs " pleine échelle », reproduisant très fidèlement l'environnement de travail, et pour le dimensionnement des besoins en ressources humaines nécessaires à la formation des professionnels (formateurs compétents, temps prévus dans les effectifs en vue de la formation). Investissement et obligation de ces formations reflètent une véritable politique institutionnelle reposant sur des bases sécuritaires (les accidents deviennent inacceptables pour le public) et économiques. Des limites sont cependant reconnues par la plupart des professionnels, en particulier le fait que l'apprentissage ne peut être réalisé en totalité sur simulateurs mais que la pratique réelle reste nécessaire, l'expérience seule permettant de détecter les " signaux faibles ».

L'amélioration du savoir-faire grâce à la simulation est indiscutable de même que l'analyse et la modification des comportements, tout particulièrement en situation de crise. En revanche, certaines questions demeurent sans réponse : la formation par simulation diminue-t-elle les risques aéronautiques ou les 
incidents dans les centrales nucléaires ? Existe-t-il une corrélation entre formation par cette méthode et la performance ? Est-il justifié d'évaluer les professionnels par ces techniques ?

Il est cependant possible de dire, avec René Amalberti (30) que « la simulation professionnelle n'est pas un jeu. Elle donne à voir les compétences, les échecs, questionne l'ego des professionnels, et peut susciter une émotion réelle des participants. Ces dimensions peuvent servir utilement l'acquisition des compétences, mais leur gradation doit être particulièrement contrôlée, et probablement appliquée avec discernement selon les personnalités des opérateurs. Car l'opérateur de conduite en formation est bien la cible du dispositif ; et c'est bien aussi le seul élément du puzzle qui ne soit pas simulé [...] ».

\section{Enseignements tirés des méthodes de simulation en dehors de la santé}

$\rightarrow$ Une conviction profonde que la formation par la simulation est un facteur de sécurisation des pratiques.

$\rightarrow$ Un investissement en ressources humaines permettant de dédier du temps à l'entraînement des personnels est nécessaire.

$\rightarrow$ Une économie réalisée par la formation en simulation versus la formation en situation réelle est démontrée dans certains secteurs d'activité.

$\rightarrow$ Une obligation de revalidation périodique des compétences pour continuer d'exercer est indispensable.

$\rightarrow$ Dans certains domaines d'activité, une évaluation du bénéfice en matière de performance est possible.

\section{Simulation en santé : état des lieux}

Pour montrer tout l'intérêt et toute la place tenue par la simulation en santé, il suffit de consulter le recensement quasi exhaustif des centres de simulation dans le monde réalisé par l'équipe du Centre de simulation de Bristol (Royaume-Uni). Cette liste est consultable sur le site http://www.bmsc.co.uk/. Elle rapporte le nombre de centres « déclarés » par continent (1 160 aux États-Unis, 129 au Canada, 28 en Amérique du Sud, 274 en Europe, 8 en Afrique, 204 en Asie et 28 en Australie), leurs coordonnées et sites internet, le matériel utilisé et la date de création.

II s'agit d'une base de données très fiable et complète, même si de nombreux centres de création récente (en particulier français) n'y figurent pas.

Nous examinerons donc après une revue de la littérature plusieurs expériences internationales (Amérique du Nord et Europe) puis l'avancement de ces démarches en France.

\subsection{Données de la littérature}

\section{Simulation et disciplines médicales}

Les données existantes sur l'état des lieux international de la simulation en santé sont rarement présentes dans la littérature. Néanmoins, on retrouve plusieurs études et revues thématiques.

Une enquête publiée en 2002 (38) avait pour objectif de rassembler les informations concernant l'utilisation de la simulation pour la formation, l'évaluation et la recherche en anesthésie. Un questionnaire de soixantesept items avait été adressé à cent cinquante huit centres de différents pays pratiquant la simulation médicale. Soixante réponses reçues $(38 \%)$ dont quarante et une émanant de facultés de médecine avaient permis de préciser que $77 \%$ des centres répondeurs intervenaient dans la formation initiale et $85 \%$ dans la formation continue. Peu de ces centres étaient impliqués dans l'évaluation des compétences. L'insuffisance de ressources financières et humaines a été le critère le plus fréquemment souligné par les répondants. 
Une revue complète de la littérature (1986-2006) a été réalisée concernant la formation par la simulation en psychiatrie. Sur 5000 articles étudiés concernant la formation par la simulation des professionnels de santé, soixante-douze articles et ouvrages utilisaient le terme " simulation », " patient standardisé » ou " jeu de rôle » dans la formation en psychiatrie. Sur 900 articles concernant la validation des compétences cliniques (Examens cliniques objectifs structurés : [ECOS]), 24 articles concernaient la validation par la simulation. Les conclusions de cette revue montraient que la simulation était utilisée pour l'enseignement, l'évaluation et la recherche à tous les niveaux de formation en psychiatrie. La simulation et le recours aux patients standardisés étaient utiles et adaptés pour la formation et l'évaluation.

Ce mode de formation était tout à fait accepté aussi bien par les étudiants en formation initiale que par les professionnels en formation continue. Le jeu de rôle occupait également une place importante. L'une des conclusions portait aussi sur la nécessité de développer la recherche, afin de montrer l'impact des différentes techniques de simulation sur la formation en psychiatrie et d'en évaluer la qualité. Plusieurs travaux ont concerné la simulation chirurgicale (39).

La formation aux actes interventionnels consiste à atteindre des objectifs cliniques, techniques et comportementaux. Le compagnonnage demeure le principal mode d'entraînement pour cette formation, mais la nécessité de répéter les gestes techniques en particulier a conduit à utiliser des modèles tels les animaux ou les cadavres, et plus récemment les simulateurs. La question qui se pose aujourd'hui est de savoir non seulement si ces nouvelles méthodes pédagogiques sont utiles à la formation des futurs opérateurs, mais également si elles permettent de limiter les risques pour les patients.

Quelques revues ont étudié la validité des travaux scientifiques concernant la simulation chirurgicale. Nous en retiendrons deux :

- la première, réalisée par des équipes australiennes (40) retrouve par l'intermédiaire de plusieurs bases de données (principalement Medline, Embase, the Cochrane Library, etc.) trente études randomisées contrôlées sur le sujet comportant au total 760 participants.

- les principaux résultats sont les suivants : la méthodologie utilisée est notablement insuffisante (absence de double aveugle et de suivi en particulier), petits échantillons de participants, comparaisons multiples et non précises, techniques non standardisées, etc.). Les résultats globaux ne permettent pas d'affirmer la supériorité d'une des méthodes de simulation (informatique, vidéo, matériel ou cadavre) par rapport à une formation classique ou à l'absence de formation préalable.

En conclusion, les auteurs insistent sur la nécessité de réalisation d'études multicentriques randomisées avec des protocoles communs. Le coût de la simulation doit être parallèlement étudié.

La seconde revue, réalisée par the Institute for Simulation and Interprofessionnal Studies (ISIS) (41) a retenu 83 études (pubmed). Une grande majorité de celles-ci (82\%) était monocentrique, et la moyenne des participants étudiés était de 37 . Pour les auteurs, moins de la moitié des études retenues (45\%) rapportait des données fiables. Pour les études comportant des évaluations par des observateurs (33\%), un tiers d'entre elles (34\%) n'utilisait pas de méthode en aveugle. Les résultats les plus fréquemment rapportés étaient la durée de la tâche $(86 \%)$, les erreurs techniques $(48 \%)$ et le nombre de mouvements réalisés (15\%).

\section{- Simulation paramédicale}

(Contribution du professeur Guillaume Alinier, manager du programme de simulation, centre médical et de recherche Sidra, (Doha, Qatar), et professeur en formation du personnel de santé par la simulation, université de Hertfordshire, Hatfield, Grande-Bretagne).

Les professionnels paramédicaux constituent l'effectif majeur du personnel de santé, et la plus grande partie de leur temps est passé au contact direct avec les patients, raison pour laquelle il est important de mettre à leur disposition les meilleurs moyens de formation possibles, que ce soit de façon unidisciplinaire ou multidisciplinaire. 
Les efforts de recherche et de développement en simulation médicale au cours de la fin du siècle dernier ont été dirigés par le corps médical (42), principalement dans le domaine de l'anesthésie (43-45) et de la cardiologie (46).

Dès 1971, cependant, des modifications furent apportées au premier simulateur de patient afin d'étendre son utilisation à d'autres professionnels de la santé (11). À cette époque, un nombre très limité de personnes avait alors l'opportunité de prendre part à des sessions de simulation.

De nos jours, on observe une nouvelle émergence de l'utilisation des méthodes de simulation pour le personnel paramédical en formation initiale comme en formation continue. Au Royaume-Uni, la formation des élèves infirmiers est constituée de 2300 heures de pratique (contact avec des patients) sous la forme de stages en hôpital, ainsi que de 2300 heures de formation théorique en établissement universitaire, incluant les sessions pratiques en laboratoire. Ce modèle de formation fait qu'il y a une demande permanente de places de stage, et que leur qualité éducationnelle varie grandement en fonction du service et du support donné par les superviseurs, le manque d'opportunités de stages hospitaliers de qualité (47) et la recommandation de reconnaître les heures de formation par la simulation comme une méthode de formation à part entière (48). Le Nursing and Midwifery Council (NMC) a d'ailleurs lancé un appel à projets de recherche universitaire (49).

Le NMC a accompagné treize projets de relativement courte durée (trois mois), de nature très diverse en termes de pédagogie et de simulation, et sans support financier. Informé des résultats des divers projets de recherche menés par différentes universités $(48,50,51)$, le NMC a publié une circulaire officielle stipulant qu'un maximum de 300 heures de formation pratique hospitalière pouvaient maintenant être acquises par le biais de la simulation en laboratoire universitaire (52). Cependant, cette dérogation ne donne aucune définition du terme " simulation », mais stipule simplement que ces heures de formation pratique doivent s'effectuer dans un environnement clinique simulé. Bien que la plupart des universités n'utilisent pas encore leur quota de 300 heures de simulation, quelle qu'en soit la forme (basse ou haute-fidélité), il y a eu une plus grande prise en compte de l'importance des divers types d'approches éducationnelles à la formation des élèves infirmiers. Certains états d'Amérique du Nord ont adopté une approche similaire en reconnaissance de la valeur éducationnelle de l'expérience acquise au travers de la simulation $(53,54)$.

La prise en charge des patients est un travail d'équipe et bien que chaque profession ait sa spécificité, il est maintenant reconnu qu'un certain degré de formation en commun est requis afin d'assurer le fonctionnement optimum d'une équipe $(55,56)$. Cela permet d'augmenter les opportunités de formation multiprofessionnelles tant en formation de base qu'en formation continue (57-59).

Dans l'état de l'Oregon, il est intéressant de noter que la stratégie d'adoption globale de la simulation dans le secteur de la santé a commencé par le domaine paramédical, car la simulation y est plus communément utilisée que dans le domaine médical (60). Certains hôpitaux aux États-Unis ont aussi adopté la simulation pour l'orientation des nouveaux infirmiers (61), dans des programmes de formation du second cycle universitaire (62). L'objectif est de mieux les préparer à l'activité clinique en les confrontant à des cas complexes en simulation impliquant d'autres professions, mais aussi en formation continue, afin de juger les compétences cliniques du personnel infirmier (63). Une étude doctorale, conduite en 2005 aux États-Unis, rapportait que d'ici $2008,88 \%$ des programmes de formation des infirmiers anesthésistes allaient inclure l'utilisation de simulateurs de patients (64). En vue de promouvoir le développement de la pratique de la simulation aux États-Unis, une association fut créée en 2001 sous l'appellation INACSL pour Internationale Nursing Association for Clinical Simulation and Learning et son journal «Clinical Simulation in Nursing » commença à être publié en 2006.

À l'échelle internationale, le potentiel éducationnel de la simulation a été reconnu et a encouragé de nombreux changements au niveau des programmes de formation dans le domaine paramédical. C'est un mode de formation apprécié autant des étudiants ou participants qualifiés que des formateurs, car cela leur 
permet d'identifier leurs limites et lacunes (65). Cependant, ces mêmes articles ainsi que d'autres rapports soulignent l'importance de la formation spécifique des formateurs qui utilisent la simulation, de leur personnalité et du soin devant être apporté à l'intégration et la qualité de ce type de formation, afin que cela soit bénéfique aux participants (66-69).

\section{Simulation et recherche}

Plusieurs revues mettent en évidence les faiblesses méthodologiques des travaux de recherche concernant la simulation en santé $(41,70)$. Les causes en sont nombreuses et non limitatives : faible nombre de sujets inclus, puissance statistique insuffisante, grande variabilité des tests utilisés pour l'évaluation, etc.

La revue Cochrane, publiée en 2009 (71) reprenant les études contrôlées randomisée sur l'efficacité des méthodes de simulation en chirurgie, concluait sur la nécessité d'une plus grande rigueur méthodologique dans les travaux entrepris. Van Nortwick et al. (37) ont proposé des recommandations pour une standardisation méthodologique des travaux de recherche sur le thème de la simulation.

Il a par ailleurs été proposé que les techniques de simulation soient utilisées pour les essais cliniques de phase 3. L'incorporation de la simulation au sein des protocoles aurait de nombreux avantages : diminution des violations de protocole, augmentation du nombre de participants inclus, augmentation de la sécurité des participants, etc.(72). Enfin, selon Taekman et al., la formation des coordonnateurs d'études cliniques pourrait être notablement améliorée par la simulation. Ces travaux demandent cependant d'être confirmés (73).

\subsection{Simulation en Amérique du Nord}

(Contribution rédigée par Sylvain Boet MD, Med*, Viren N. Naik MD, FRCPC, Med Allan Waters Family Patient Simulation Centre, St. Michael's Hospital, University of Toronto, Toronto, Ontario, Canada, Department of Anesthesiology, The Ottawa Hospital, University of Ottawa, Ottawa, Ontario, Canada \& The Royal College).

Le but du texte suivant est de fournir une synthèse de l'état des lieux et de l'évolution de la place de la simulation en santé en Amérique du Nord $(74,75)$.

La simulation est largement intégrée dans l'enseignement des disciplines de santé en Amérique du Nord. Elle est utilisée de façon routinière en particulier dans les formations médicales, chirurgicales, paramédicales (infirmières, ambulanciers, kinésithérapeutes) et aussi de diététique, de pharmacie. La grande majorité des établissements de formation en santé dispose d'un programme de simulation. II est intéressant de noter la distinction entre un programme en simulation et un centre de simulation. Un programme de simulation signifie que la simulation est utilisée comme outil d'éducation, alors qu'un centre de simulation correspond à un lieu physique dédié spécifiquement à l'enseignement par simulation. La distinction est intéressante, car il est possible de disposer d'un programme de simulation accrédité sans disposer de centre de simulation à proprement parler.

Le premier centre de simulation canadien a ouvert en 1995 à Toronto, peu de temps après les premiers centres aux États-Unis. II existait une demi-douzaine de centres de simulation en 1999, plus de soixante lors du dernier recensement en $2009^{5}$. Ceci correspond à environ quatre-vingts programmes de simulation d'après les estimations actuelles. Beaucoup de centres de simulation se situent dans les hôpitaux, d'autres sont dans les institutions de formation, notamment paramédicales. Aujourd'hui, les établissements de soins et de formation ne disposant pas de centre de simulation, sont considérés comme peu attractifs aussi bien par les patients que les étudiants. Les centres de simulation sont donc aussi une vitrine pour la promotion de l'excellence des établissements de soins et de formation. Après la phase naturelle de construction de centres, la priorité a été de coordonner les différents centres en réseaux, avec l'objectif d'optimiser les ressources : c'est par exemple un des objectifs du Network of Excellence in Simulation for Clinical Teaching and Learning de Toronto, Canada.

5. Données non publiées, fournies gracieusement par le Collège royal des médecins et chirurgiens du Canada. 
Les centres de simulation ont plusieurs types d'activités. Leur mission première est l'enseignement, principalement pour la formation initiale et, depuis plus récemment, pour la formation continue. Tous les types de savoir sont enseignés : les gestes techniques comme les compétences relationnelles. Mais la simulation est aussi utilisée pour beaucoup d'autres activités.

En effet, la formation des personnels de santé, lors d'introduction de nouveaux matériels ou nouvelles techniques de soins (en chirurgie par exemple), est de plus en plus assurée grâce à des simulateurs avant la mise en place clinique auprès des patients.

La simulation est aussi utilisée pour développer la formation et la recherche en éducation médicale ainsi que dans le domaine en expansion constante des facteurs humains et des compétences non techniques telles que la communication et le travail en équipe (76).

Un des axes de recherche actuel est celui de l'optimisation des ressources pour l'enseignement par simulation (77). Le développement actuel de la simulation interprofessionnelle est majeur, même si de nombreuses barrières persistent concernant son utilisation routinière.

Enfin, un autre axe de développement important est celui de la certification et recertification des professionnels de santé. La certification (obtention du diplôme de formation initiale) est déjà utilisée régulièrement pour les ambulanciers et autres professions paramédicales. La simulation, notamment grâce à des patients standardisés, a une place pour l'instant limitée pour la validation des compétences des professions médicales, mais devrait s'accroître au cours des prochaines années (77).

La simulation a aussi été introduite pour la recertification (autorisation d'exercice soumise à une revalidation régulière). Par exemple, les anesthésistes des États-Unis doivent participer à une séance de formation par simulation (programme MOCA) à chaque cycle de recertification. Pour le programme MOCA, il s'agit pour l'instant d'une évaluation formative (la simple participation est validante), non sommative (pas d'examen sur simulateur) pour l'instant.

Le financement des programmes et centres de simulation a été principalement public. Dans un second temps, des centres privés ont été créés principalement aux États-Unis, alors que le Canada a privilégié les partenariats privé-public.

Le modèle économique des centres publics repose sur le principe d'autofinancement par les formations, les donations et la recherche, une fois la phase de lancement initiale passée. Le personnel, spécifiquement dédié au fonctionnement des centres de simulation, est primordial ; il optimise la valeur ajoutée de chaque intervenant dans les formations. Le coût en personnel tel que les techniciens en simulation et les formateurs ne doit pas être sous-estimé et peut représenter plus de la moitié du budget de fonctionnement (78).

Les programmes de formation notamment recertificatifs sont une source de revenus non négligeable pour les centres de simulation et contribue en partie à leur autofinancement. Les assureurs des professions médico-chirurgicales proposent depuis plusieurs années aux États-Unis des réductions significatives de primes d'assurance pour les praticiens justifiant de formation sur simulateurs. Au Canada, les leviers de financement sont au contraire majoritairement publics.

L'accréditation des centres de simulation est en place aussi bien aux États-Unis qu'au Canada avec des systèmes et philosophies différents. Aux États-Unis, plusieurs organismes ont la possibilité de certifier les formations dispensées par les centres de simulation : la société internationale de simulation en santé, la société américaine des anesthésistes, la société américaine des chirurgiens, etc. Chaque organisme accréditeur est en mesure de certifier une formation particulière au sein d'un centre de simulation donné. 
À l'opposé, au Canada, un seul organisme accréditeur (le Collège royal) accrédite chaque programme de simulation pour l'ensemble des formations dispensées par ce centre. Ce processus d'accréditation des centres de simulation inclut l'accréditation des formateurs. Évidemment, les centres ne peuvent offrir des programmes de formations recertificatives qu'après avoir obtenu leur accréditation.

En conclusion, la simulation est partie intégrante du monde de la santé en Amérique du Nord. La simulation est un des éléments essentiel de la formation de toutes les professions de santé et est de plus en plus utilisée à visée (re)certificative.

\section{En Amérique du Nord : la simulation est un outil pédagogique à part entière}

$\rightarrow$ Elle est utilisée de manière routinière dans l'enseignement initial des professions médicales et paramédicales.

$\rightarrow$ II faut distinguer programme de simulation et centre de simulation (lieu où se déroule la formation).

$\rightarrow$ La formation par simulation représente un argument d'attractivité des établissements de santé.

$\rightarrow$ L'organisation des centres se fait en réseau pour optimiser les ressources.

$\rightarrow$ La simulation est utilisée pour la certification ou la recertification des professionnels de santé.

$\rightarrow$ Une accréditation des centres est possible au moyen de référentiels validés.

\subsection{Simulation en Europe}

L'analyse de la simulation en Europe a été réalisée au moyen d'une enquête sur les centres de simulation médicale pédiatrique et l'analyse détaillée de neuf centres de simulation représentatifs de huit pays européens.

\subsubsection{Enquête européenne et simulation pédiatrique}

Une enquête européenne concernant la simulation médicale pédiatrique a été publiée en 2009 (56). Parmi les cent dix centres de simulation européens répertoriés, trente-huit avaient une activité pédiatrique spécifique. Un questionnaire de quarante items avait été élaboré et adressé à ces centres. Vingt centres $(52 \%)$ ont répondu à tous les items. Parmi eux, dix-huit avaient plus de deux années d'expérience en simulation pédiatrique, et seize étaient directement rattachés à une faculté de médecine. Les participants étaient surtout des étudiants (35\%), des internes (21\%), mais aussi des médecins seniors $(7 \%)$ et des infirmiers (20\%). Pour les externes et les internes, la formation était dans la plupart des cas obligatoire.

La formation continue était cependant réalisée dans $80 \%$ des centres, et $40 \%$ réalisaient des évaluations par cette méthode. II faut noter l'hétérogénéité géographique des centres : $50 \%$ des centres répondeurs étaient situés au Royaume-Uni, pays particulièrement en avance sur les méthodes de prévention des risques liés aux soins.

\subsubsection{Questionnaires d'enquête : centres de simulation européens}

L'analyse de l'activité en Europe a été réalisée à travers un échantillon de neuf centres européens. Les données ont été recueillies via le site internet http://www.bmsc.co.uk/ et la banque de données du SESAM. Le premier site recense l'ensemble des centres européens de simulation. L'objectif de cette étude est de donner un aperçu des centres européens, qui par leur ancienneté et leurs moyens se différencient notablement des centres français. Les résultats de cette enquête pourraient permettre d'alimenter les propositions à réaliser pour le développement des centres français. 


\section{Centres étudiés}

Huit centres sont publics et un centre est privé. La moitié est rattachée à un CHU, deux à l'université, une est une fondation et un est rattaché à la région.

\begin{tabular}{|l|l|}
\hline Bologne & Italie \\
\hline Copenhague & Danemark \\
\hline Gjovik & Norvège \\
\hline Genève & Suisse \\
\hline Santiago de Compostela & Espagne \\
\hline Stavenger & Norvège \\
\hline Tubingen & Allemagne \\
\hline Université de Hertfordshire & Royaume-Uni \\
\hline Swindon & Royaume-Uni \\
\hline
\end{tabular}

$\rightarrow$ La moitié des centres a été créée avant 2001. La région de Galice en Espagne développe une politique nationale (cf. « Rapport NHS »).

$\rightarrow$ Les centres sont dirigés dans les $2 / 3$ des cas par des médecins (deux professeurs et cinq docteurs en médecine) appartenant à l'hôpital et à l'université.

$\rightarrow$ Dans certains centres, on trouve des managers disposant de compétences purement administratives (type directeur d'hôpital) ou en sciences de l'éducation. Lorsqu'il s'agit de médecins, se sont le plus souvent des médecins anesthésiste-réanimateurs.

\section{Financement de départ}

Plusieurs centres sont financés de manière non négligeable par la clientèle externe à l'hôpital ou à l'université, mais de manière générale, les principaux financeurs sont les régions, les fonds privés de l'industrie y compris pharmaceutique et les centres hospitaliers.

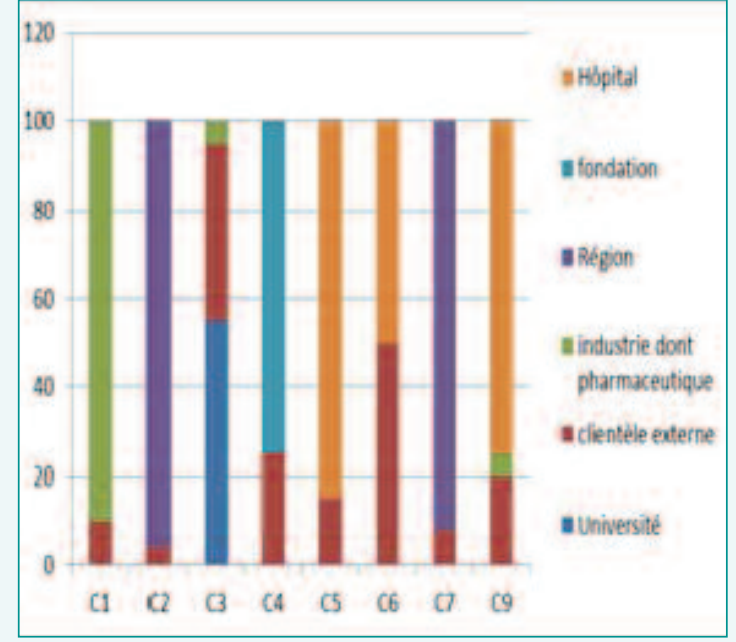

Financement de départ

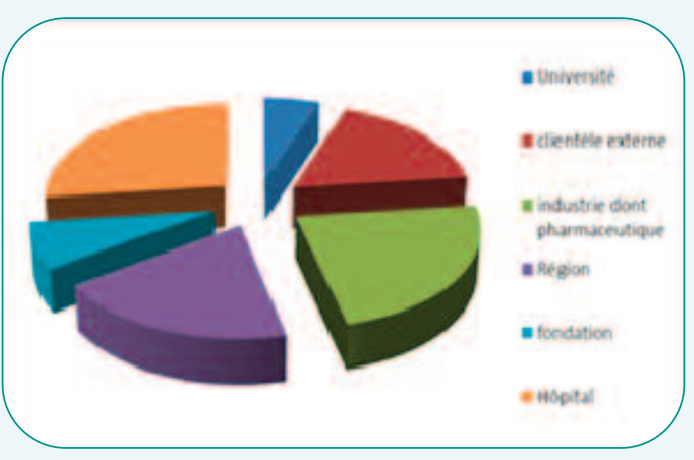

Répartition générale du financement 


\section{Surfaces allouées}

Six centres ont communiqué leur surface ; dans l'ensemble très spacieux, la médiane se situe à $245 \mathrm{~m} 2$.

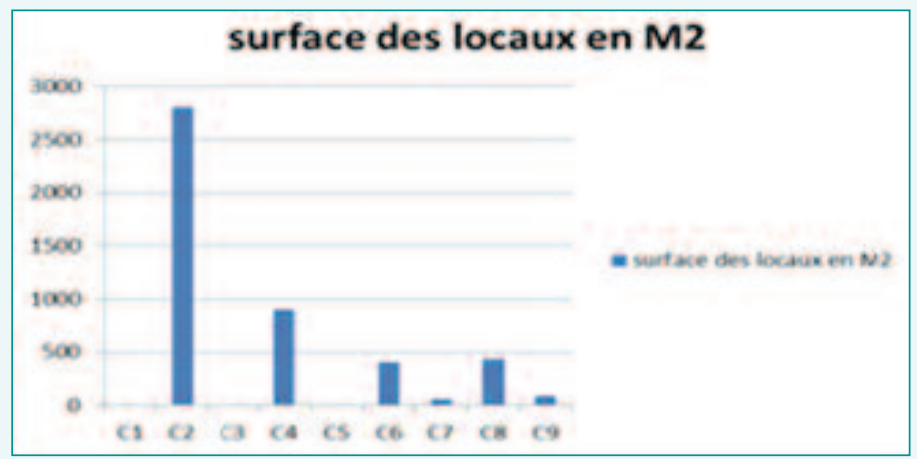

L'ensemble des centres dispose de locaux dédiés à la simulation. Le nombre de salles de simulation par centre varie de 1 à 13 , avec une médiane de 4 (versus 2 dans les centres français) et une moyenne de 4,9 (versus 2,9 en France). Les salles de simulation ont souvent une salle de contrôle dédiée.

\section{Ressources humaines des centres}

Tous les centres disposent de formateurs permanents formés, mais il est à noter la grande proportion de formateurs occasionnels (cf. graphique ci-dessus).

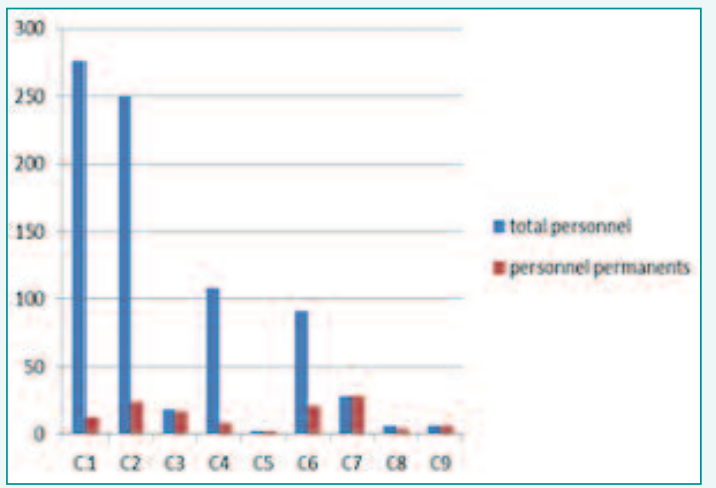

Les ressources humaines sont importantes et diversifiées puisque l'on y trouve en dehors des médecins (pédiatres, MAR, radiologues), des psychologues, des gestionnaires de risques, des IDE, des IDE spécialisées, et des cadres de santé.

$\rightarrow$ Le personnel dédié aux activités support est aussi très présent ; la plupart des centres disposent de secrétaires ou hôtesses (1 à 4), de personnel technique (1 à 4). Un centre dispose de 10 personnes ponctuellement.

\section{Activité de formation}

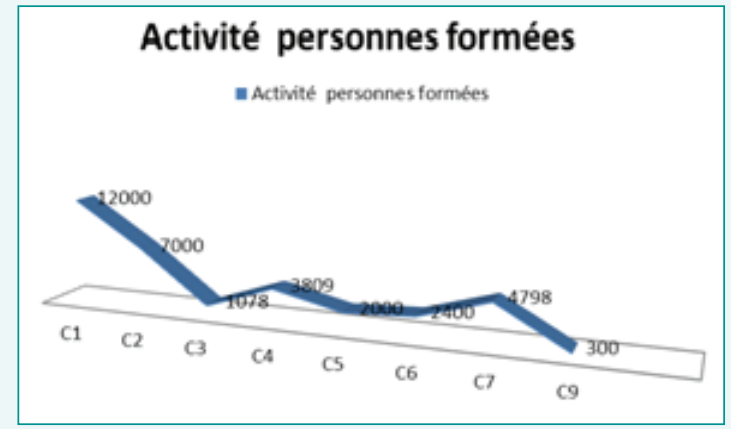

L'activité est importante même dans les centres disposant de peu de formateurs. L'activité médiane est de 3104 personnes formées par an (un centre n'a pas communiqué son activité de formation). 


\section{Types de formation}

L'ensemble des centres dispense la formation initiale et professionnelle. La priorité est donnée à la formation professionnelle. Elle concerne les hospitaliers et les professionnels extérieurs au centre hébergeant le centre de simulation.

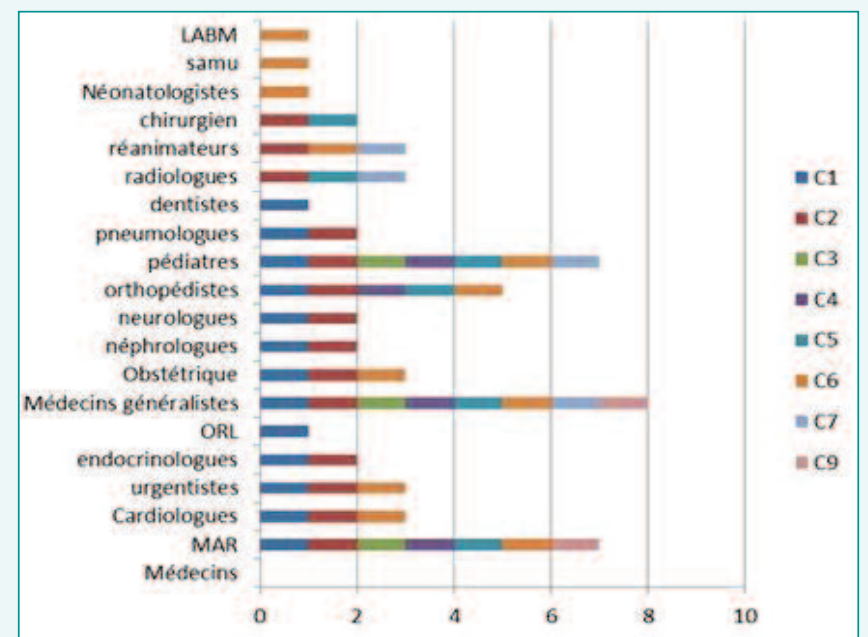

Disciplines médicales en formation continue $\rightarrow$ Les disciplines les plus enseignées sont la pédiatrie, la médecine générale, l'anesthésie réanimation, la médecine d'urgence, l'obstétrique, la cardiologie et la radiologie. Mais il existe en Europe une grande variété de disciplines enseignées aux praticiens.

$\rightarrow 2$ centres ne forment pas les infirmières

\section{Formation continue à d'autres professionnels}

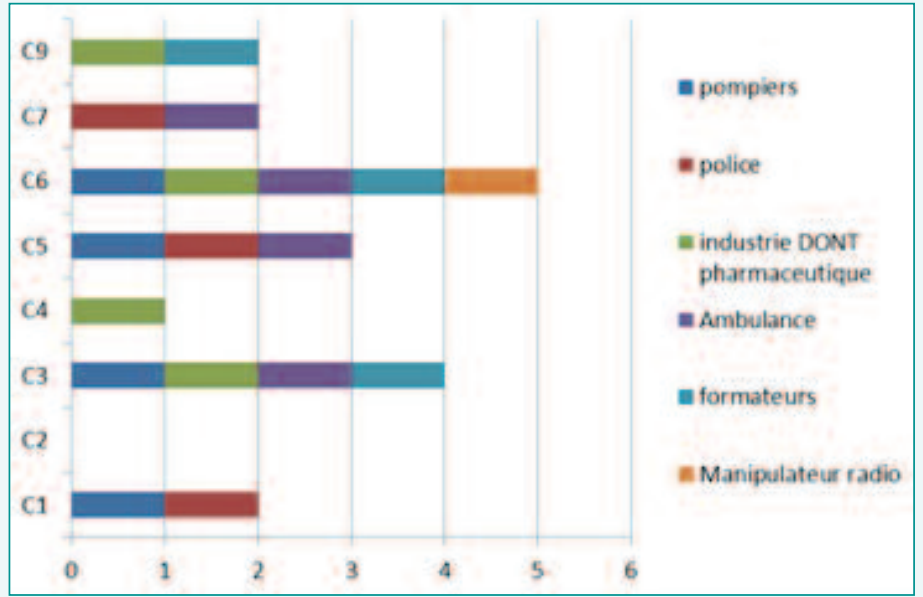

$\rightarrow$ La majorité des centres dispensent des formations à d'autres professionnels en dehors des établissements de santé, plus généralement aux pompiers (ou services de secours) et aux ambulanciers. 


\section{Équipement}

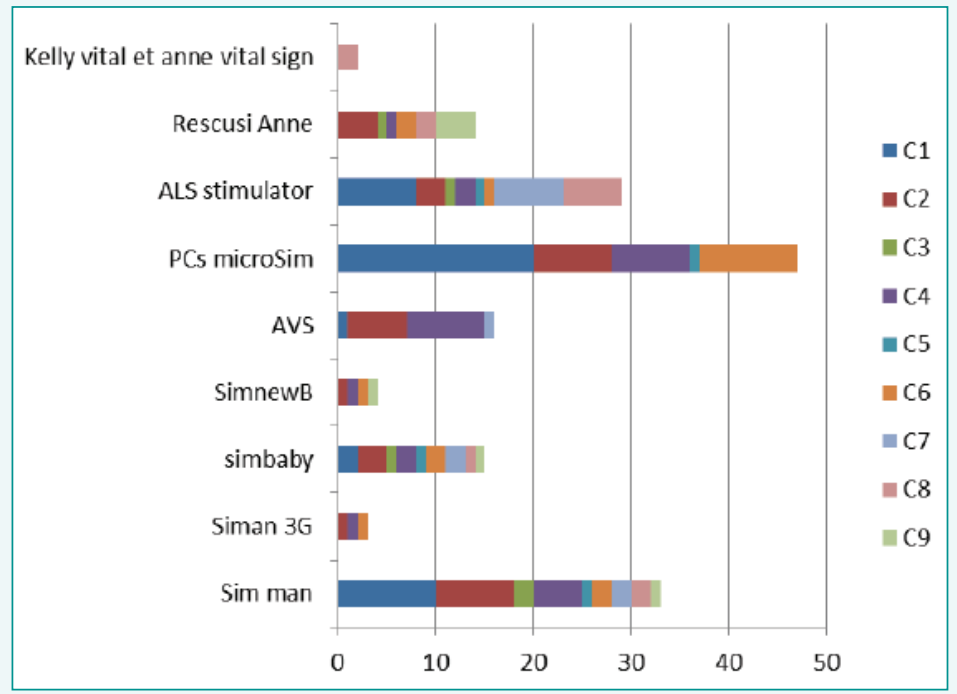

$\rightarrow$ Les centres européens sont bien équipés. Le plus souvent, ils utilisent $\operatorname{SimMan}^{\circledR}$, ALS, AVS et SimBaby $^{\mathrm{TM}}$

$\rightarrow$ La médiane se situe à treize équipements.

Total équipement par centre

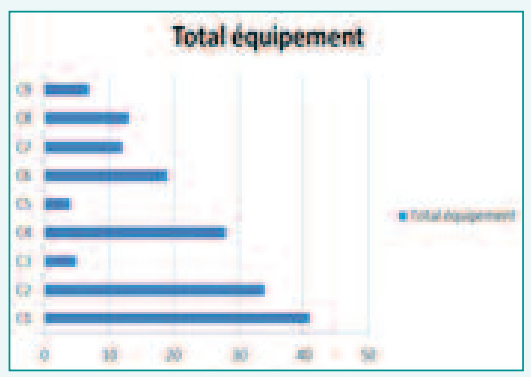

\section{Scénarios}

Tous les centres utilisent des scénarios comme support de formation.

\section{Principaux types de scénarios}

\begin{tabular}{|l|l|}
\hline Arrêt cardio-respiratoire & Coma \\
\hline Douleur thoracique & Complication anesthésique \\
\hline Choc hémorragique & Intubation difficile \\
\hline Infarctus du myocarde & Anaphylaxie \\
\hline Traumatismes & Debriefing d'accident \\
\hline Épilepsie & Réanimation néonatale \\
\hline Consultation d'annonce de mauvaise nouvelle & Complications néonatales \\
\hline
\end{tabular}

\section{Recherche}

Huit centres sur neuf ont des activités de recherche et de publication très importantes portant particulièrement sur : le transfert de compétence, la gestion des situations de crises, l'évaluation de l'impact de la simulation sur la formation versus la formation classique, l'intérêt de la simulation pour améliorer la sécurité des soins en particulier dans les situations d'urgences, les méthodes de validation des scénarios, la validité écologique des scénarios, les facteurs humains, les analyses d'accident, la simulation et formation en soins infirmiers, etc. 
En Europe : la simulation est un outil pédagogique bien implanté, mais les inégalités sont importantes

$\rightarrow$ Le développement est plus récent qu'en Amérique du Nord.

$\rightarrow$ L'investissement de départ est approprié (locaux, ressources humaines, matériel) pour la majorité des centres.

$\rightarrow$ Une part de financement importante des investisseurs privés et de la communauté européenne existe.

$\rightarrow$ Une activité de formation professionnelle continue demeure majoritaire.

$\rightarrow$ Toutes les disciplines sont envisagées avec une ouverture à d'autres secteurs que la santé.

$\rightarrow$ Une activité de recherche en simulation croissante.

II est important de considérer également, pour l'état des lieux européen, les éléments du rapport du NHS anglais, qui sert de base à la politique du Royaume-Uni originale en matière de simulation.

\subsection{Rapport du NHS anglais (79)}

Le département de la santé du Royaume-Uni a commandité un rapport concernant l'organisation et le financement de la simulation. Celui-ci, remis en mars 2010, faisait suite aux conclusions du rapport annuel 2008 du Chief Medical Officer (CMO) (80) déclarant que la formation par la simulation, sous toutes ses formes, devait jouer un rôle essentiel dans l'élaboration d'un système de soins plus sûr.

\section{Plusieurs points essentiels étaient abordés au sein de ce rapport}

\section{Leadership et vision stratégique}

II existe des variations régionales notables. La plupart des centres étudiés ont des objectifs cliniques, certains une approche d'équipe ; très peu utilisent la réalité virtuelle (considérée comme coûteuse et d'utilité discutable).

Cette diversité a entraîné le développement de "visions » locales de la simulation. Certaines régions sont très bien organisées (Londres), tant au plan des objectifs pédagogiques que de l'organisation des moyens et de la recherche. D'autres régions ont des approches moins claires et souvent dépendantes de personnalités locales.

Cependant, la plupart des régions considère la simulation comme une priorité. Leurs avis sur l'organisation divergent. Beaucoup sont en faveur d'une centralisation des moyens, mais obligent les apprenants à se déplacer. Certains sont en faveur d'une centralisation, mais avec la possibilité de délocalisation (structures mobiles). D'autres enfin sont favorables à l'apprentissage au plus près du lieu de travail. Toutefois, tout ceci est lié aux moyens financiers et humains (formateurs) disponibles.

Actuellement, il existe des inégalités importantes d'accès à la simulation au Royaume-Uni. Si des divergences persistent concernant l'organisation globale, un consensus fort se fait sur la nécessité d'attribution de moyens suffisants pour un accès équitable.

\section{$\rightarrow$ Financement des centres}

La généralisation des simulateurs haute-fidélité (HF) est largement dépendante des moyens financiers. Les mesures de protection qui entourent les simulateurs peuvent limiter l'accès des apprenants dans ces centres. 
En pratique, certains centres sont très actifs avec des moyens limités tandis que d'autres, avec des moyens importants travaillent peu. Cet usage " suboptimal » est souvent lié à un manque de personnel formé ou à un défaut de formation de ceux-ci quand ils sont en nombre suffisant. Ceci peut représenter une sousutilisation importante des ressources qui souligne la nécessité de la création d'un réseau pour certaines régions amenant au développement d'équipes locales spécialisées.

Une plus grande transparence des financements (pour la simulation, mais aussi pour l'éducation en général) apparaît nécessaire et est réclamée par beaucoup d'équipes (financement transparent, adéquat et équitable). Certains centres sont contraints de faire payer leurs formations en l'absence de subvention. Ces éléments ne peuvent faciliter la création de réseaux. En outre, le « suivi financier » manque souvent de professionnalisme.

\section{$\rightarrow$ Expertise des formateurs : une nécessité absolue}

Dans plusieurs centres, le temps consacré à l'enseignement en général et à la simulation en particulier est notablement insuffisant et rarement intégré dans les plannings. Ces activités nécessitent du temps et des moyens pour les enseignants.

La formation des experts est essentielle tout particulièrement en ce qui concerne les techniques de debriefing. Cette formation doit être validée au plan universitaire. De même, l'élaboration de programmes de formation est indispensable, et nécessite des connaissances avancées en simulation. Enfin et surtout le rapport insiste sur le fait que la simulation est une méthode d'enseignement parmi d'autres et n'est pas une fin en soi. Elle doit être intégrée dans les programmes généraux de formation initiale et continue.

\section{$\rightarrow$ Formation à la sécurité de soins}

L'apprentissage par simulation contribue à l'amélioration des connaissances, à une meilleure rétention de celles-ci et à une modification positive des comportements. Il est maintenant démontré que les techniques de simulation contribuent à la sécurité des soins (39,81-90). La simulation permet en outre de limiter l'impact de la directive européenne sur la limitation du temps de travail des professionnels de santé, en lien direct avec la durée de formation et donc la sécurité des soins.

Des travaux de recherche sont cependant nécessaires pour valider définitivement l'efficacité de l'enseignement par simulation, tant au niveau des individus qu'au niveau des équipes.

\section{Le rapport objective certains obstacles à la mise en œuvre optimale de la formation par} la simulation en santé

$\rightarrow$ Manque de transparence dans l'obtention et la gestion des financements.

$\rightarrow$ Priorisation des activités cliniques par rapport aux activités de formation.

$\rightarrow$ Mise en place de la directive européenne sur la limitation du temps de travail.

$\rightarrow$ Non-reconnaissance du travail des formateurs.

$\rightarrow$ Écoute insuffisante des demandes des patients.

\section{5 État des lieux en France en matière de simulation en santé}

\subsubsection{Objectifs de l'étude}

Comme cela a été précisé, la simulation en santé est une activité émergente en France. Il est donc important de connaître à la fois le niveau de connaissance de ces méthodes et l'attrait qu'elles suscitent 
de la part des professionnels de santé. En effet, il est intéressant de savoir si des méthodes de simulation ou apparentées à la simulation sont déjà utilisées par les professionnels de santé. Dans ce cas, la nature et la diversité des champs couverts permettraient d'évaluer s'il existe un a priori favorable à ce type de formation, voire un véritable besoin, et donc d'identifier le paysage de la simulation française à la fois topographique et taxonomique. L'étude a donc été réalisée par la mission simulation au travers de deux enquêtes. Dans un premier temps, elle a été très ouverte et exclusivement descriptive, permettant d'identifier les champs couverts et ceux à développer. Elle a permis d'évaluer la demande potentielle en matière de plates-formes de formation par la simulation et d'identifier les outils utilisés.

Le deuxième temps de l'étude s'est attaché à évaluer le nombre de structures formalisées de formation par la simulation et leur répartition géographique. Elle a permis de connaître l'offre de formation ainsi proposée et d'en évaluer le niveau de qualité à la fois sur le plan de la structure, des moyens alloués et des programmes de formation. Les résultats devraient donc permettre de mesurer le niveau d'avancement français dans ce domaine.

\subsubsection{Méthodologie}

Différentes méthodes ont été utilisées :

- un questionnaire d'enquête simplifié ayant pour but de repérer les activités de simulation dans les différents types d'établissements de santé et les écoles. Ce questionnaire a été complété par une recherche internet sur les diplômes universitaires en santé, utilisant la simulation comme outil pédagogique.

- un questionnaire d'enquête complet destiné à préciser l'organisation et le fonctionnement des centres de simulation installés ou en cours d'installation.

\section{- Questionnaire simplifié de repérage des activités de simulation}

\section{$\rightarrow$ Échantillon}

Le questionnaire a été adressé à un échantillon représentatif des établissements de santé d'une taille considérée comme suffisante pour engager des moyens de formation de type simulation :

- les établissements publics de plus de 500 lits ;

- les établissements privés de plus de 250 lits ;

- l'ensemble des centres de lutte contre le cancer ;

- les établissements PSPH de plus de 250 lits.

Bien que le champ de l'étude reste le DPC, ce questionnaire a également été adressé aux facultés de médecine et de pharmacie, aux instituts de formation en soins infirmiers (IFSI) et aux écoles de sagesfemmes, afin d'identifier si la culture simulation existe en amont du DPC, mais aussi si les facultés et écoles développent des programmes de formation postuniversitaires dans ce domaine.

\section{$\rightarrow$ Items du questionnaire}

Trois questions ouvertes ont été proposées :

- votre établissement réalise-t-il des formations par la simulation?

- si oui décrivez-les brièvement ;

- communiquez les coordonnées des personnes en charge de la formation.

Une plaquette décrivant brièvement les différentes méthodes de simulation était jointe au questionnaire afin que chaque établissement puisse se représenter ce qui dans sa structure pouvait être considéré comme méthode de formation par la simulation (cf. Annexe 3). 


\section{Modalité de tri des réponses}

Compte tenu de la grande diversité des réponses, celles-ci ont été triées en premier lieu selon la technique de simulation utilisée (Tableau 1) puis selon le type d'activité mis en simulation (Tableau 2).

Tableau 1. Modalité de classement des techniques de simulation citées par les répondants

\begin{tabular}{|l|l|}
\hline Techniques de simulation & Exemples \\
\hline Haute-fidélité avec matériel & Mannequins pilotés par informatique \\
\hline Haute-fidélité avec environnement réaliste & $\begin{array}{l}\text { Situation de consultation simulée } \\
\text { Reconstitution d'une officine }\end{array}$ \\
\hline Simulation procédurale & $\begin{array}{l}\text { Apprentissage de technique : pose de perfusion, de voie veineuse } \\
\text { centrale, intubation }\end{array}$ \\
\hline Mise en situation & Jeux de rôles, études de cas \\
\hline Expérimentation animale & Exercice de suture sur animal \\
\hline
\end{tabular}

Tableau 2. Modalité de classement des activités de simulation citées par les répondants

\begin{tabular}{|l|l|}
\hline Activités de simulation & Exemples \\
\hline Activités par disciplines & Simulation chirurgicale, obstétricale, cancérologique \\
\hline Activités de soins transversales & Lutte contre la douleur, éducation thérapeutique \\
\hline Activités techniques et logistiques & Simulation incendie, simulation de pannes \\
\hline Activités support, management et qualité & $\begin{array}{l}\text { Simulation d'entretiens de recrutement, d'entretiens d'audit, de plan de } \\
\text { secours }\end{array}$ \\
\hline Autres & Serious game \\
\hline
\end{tabular}

\section{$\rightarrow$ Complément à l'enquête simplifiée}

Pour mieux cerner la contribution de l'université à la formation par la simulation, quelques exemples de DU utilisant la simulation comme outil pédagogique sont rapportés dans les résultats.

\section{- Questionnaire d'enquête complet à l'intention des centres de simulation existant ou en cours de constitution (Annexe $\mathrm{n}^{\circ} 7$ )}

La liste de ces centres a été fournie par l'AFSARMU (Association Francophone de Simulation en Anesthésie Réanimation et en Médecine d'Urgence).

Ce questionnaire est composé de plusieurs chapitres concernant la structuration des centres de simulation :

- le centre et sa gouvernance ;

- les ressources humaines (formateurs permanents ou ponctuels, professionnels support) ;

- les ressources matérielles (locaux, équipement);

- les modalités de financement et les coûts ;

- les programmes pédagogiques ;

- l'offre de formation ;

- l'activité de recherche.

L'ensemble des questionnaires a été traité par méthode informatisée sous licence Sphinx. 


\subsubsection{Résultats du questionnaire simplifié (Tableau 3)}

Au total, 1213 questionnaires ont été envoyés. Trois cent six établissements ont répondu et 275 réponses ont pu être exploitées, soit un taux de réponse de $22,7 \%$.

Parmi ces questionnaires :

- 836 ont été adressés aux établissements de santé ; 200 réponses ont été obtenues, dont 174 sont exploitables soit un taux de $20,8 \%$;

- 378 ont été adressés aux écoles ; 106 réponses ont été obtenues, dont 101 sont exploitables, soit un taux de $26,7 \%$.

On peut ainsi considérer que plus de 174 établissements et 101 écoles mettent en œuvre des techniques de simulation.

En revanche, on ne peut en déduire que le nombre des non-répondants correspond à ceux qui ne pratiquent pas de simulation. L'absence de réponse correspondant soit à un manque d'intérêt pour l'enquête, soit à la difficulté d'identifier la simulation comme outil de formation.

Tableau 3. Questionnaire d'enquête simplifié : taux de réponses

\begin{tabular}{|c|c|c|c|c|c|c|}
\hline & & $\begin{array}{l}\text { Nombre de } \\
\text { sollicitations }\end{array}$ & $\begin{array}{c}\text { Nombre } \\
\text { d'établissements } \\
\text { répondants }\end{array}$ & $\begin{array}{c}\text { Taux } \\
\text { de réponse } \\
(\%)\end{array}$ & $\begin{array}{l}\text { Nombre de } \\
\text { réponses } \\
\text { exploitables }\end{array}$ & $\begin{array}{c}\text { Taux de } \\
\text { réponse } \\
\text { exploitable }(\%)\end{array}$ \\
\hline & $\mathrm{CHU}$ & 27 & 19 & 70,3 & 19 & 70,3 \\
\hline 危 & $\mathrm{CH}$ & 648 & 138 & 21,3 & 115 & 17,7 \\
\hline 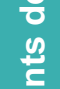 & $\begin{array}{l}\text { Assistance } \\
\text { publique }\end{array}$ & 44 & 12 & 27,2 & 12 & 27,2 \\
\hline ষ্] & CLCC & 20 & 10 & 50 & 8 & 40 \\
\hline$\frac{\frac{1}{2}}{\frac{1}{10}}$ & $\begin{array}{l}\text { PSPH/ESPIC } \\
\text { (hors CLCC) }\end{array}$ & 50 & 13 & 26 & 12 & 24 \\
\hline & Clinique & 47 & 8 & 17 & 8 & 17 \\
\hline & $\begin{array}{l}\text { Total } \\
\text { établissements } \\
\text { de santé }\end{array}$ & 836 & 200 & 23,9 & 174 & 20,8 \\
\hline & IFSI & 285 & 67 & 23,5 & 62 & 21,8 \\
\hline & $\begin{array}{l}\text { École de } \\
\text { sages-femmes }\end{array}$ & 36 & 19 & 52,7 & 19 & 52,7 \\
\hline 잉 & $\begin{array}{l}\text { Faculté de } \\
\text { médecine + } \\
\text { Faculté de } \\
\text { pharmacie }\end{array}$ & 57 & 20 & 35,1 & 20 & 35,1 \\
\hline & Total écoles & 378 & 106 & 28 & 101 & 26,7 \\
\hline & $\begin{array}{l}\text { Total } \\
\text { répondants }\end{array}$ & 1213 & 306 & 25,2 & 275 & 22,7 \\
\hline
\end{tabular}




\section{Répartition géographique des répondants}

- les résultats présentés sur la carte correspondent au nombre d'établissements répondants.

- la répartition présentée n'intègre pas les centres de simulations constitués.

- par ailleurs, au total, 1104 actions de simulation ont été recensées à travers les réponses.
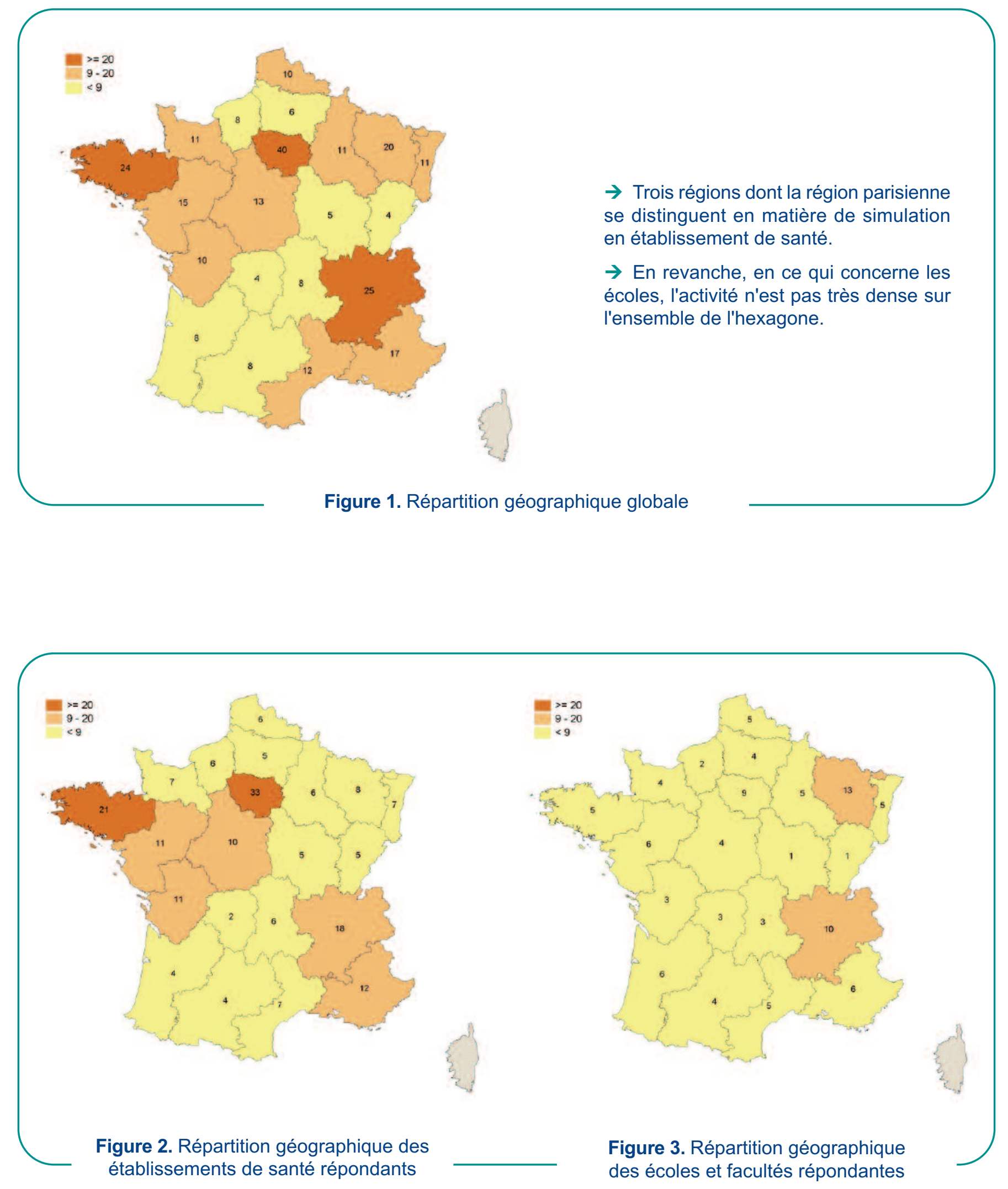


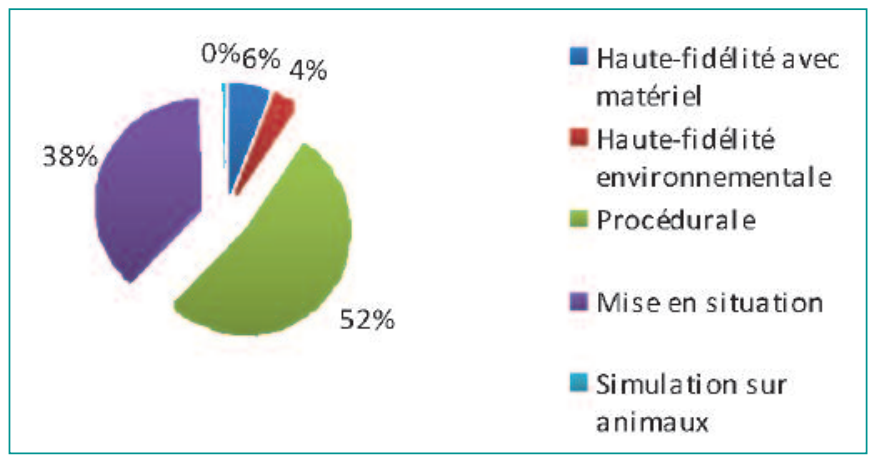

$\rightarrow$ L'analyse des techniques de simulation montre que la simulation procédurale, (l'apprentissage ciblé de techniques) et les mises en situation (études de cas ou jeux de rôles avec peu d'investissement matériel) sont les méthodes les plus régulièrement retrouvées, et cela aussi bien au sein des établissements de santé que des écoles (cf. ci-dessous).

Graphique 1. Répartition globale selon les techniques de simulation

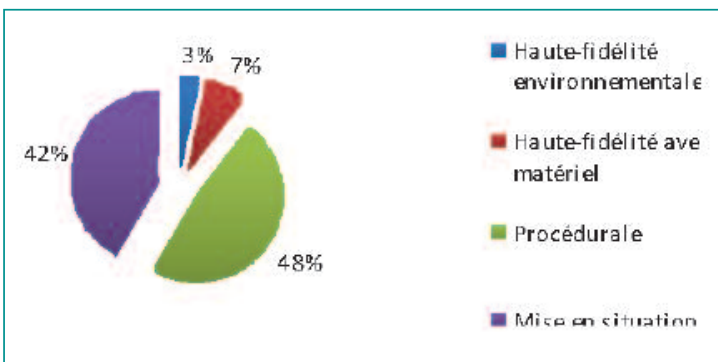

Graphique 2.a. Répartition des techniques de simulation au sein des établissements

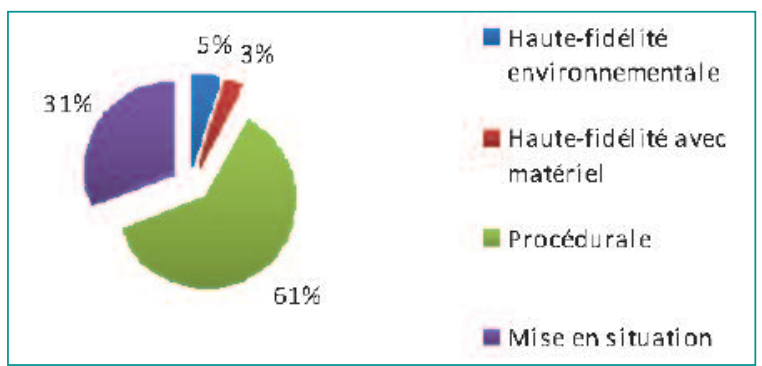

Graphique 2.b. Répartition des techniques de simulation au sein des écoles

\section{Analyse des différentes activités de simulation par type de structure}

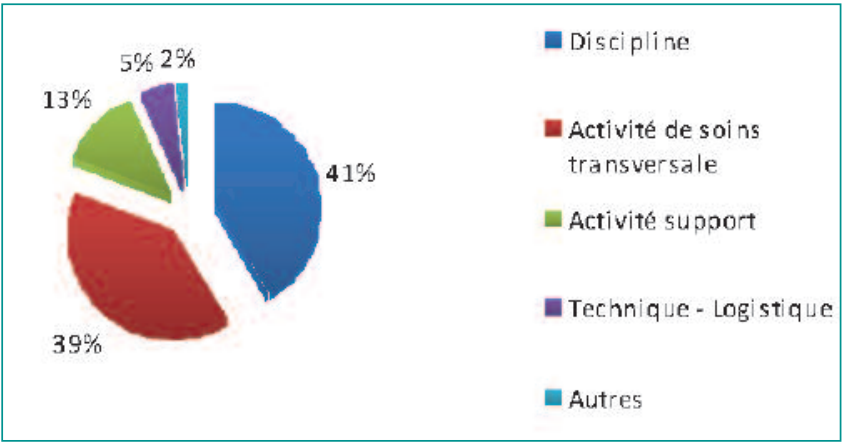

$\rightarrow$ Globalement, la majorité des activités de simulation se distribue pour partie sur les disciplines médicales et sur les activités de soins transversales. (cf. ci-contre).

$\rightarrow$ On note cependant une prédominance au sein des écoles de la simulation dans les activités de soins transversales qui correspond à l'apprentissage de gestes via la simulation procédurale (cf. ci-après). 


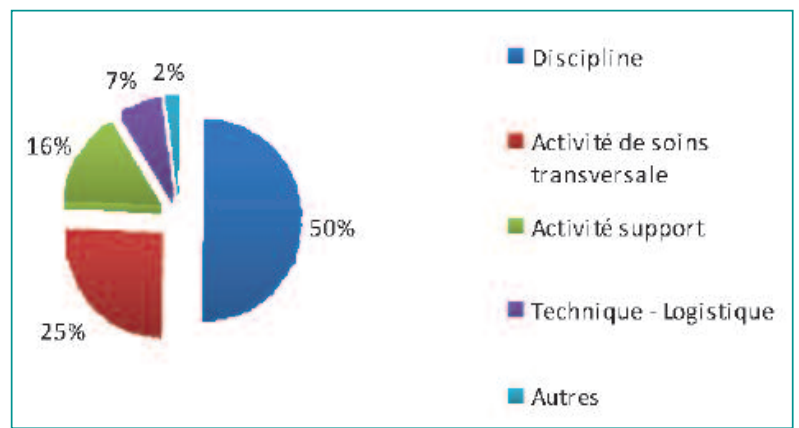

Graphique 4.a. Répartition des activités de simulation en établissements de santé

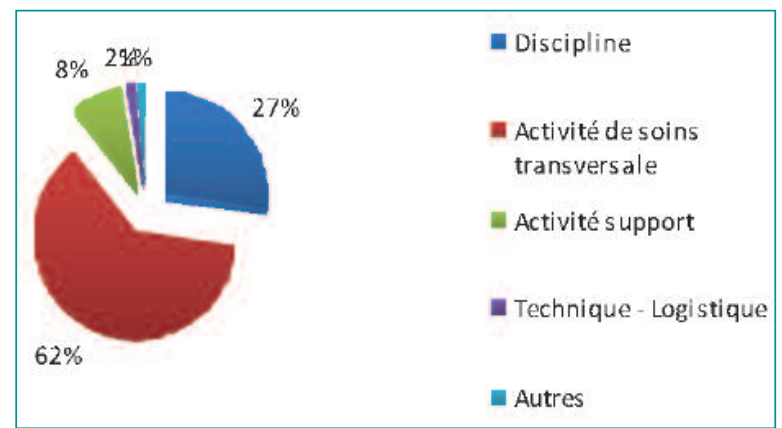

Graphique 2.b. Répartition des activités de simulation au sein des écoles

\section{Analyse de la répartition des activités par thèmes de simulations}

Tableau 4. Répartition des activités par thèmes de simulations

\section{$\rightarrow$ Disciplines}

\begin{tabular}{|c|c|}
\hline SAMU-CESU & $13,1 \%$ \\
\hline Urgences & $5,2 \%$ \\
\hline Obstétrique & $4,4 \%$ \\
\hline Anesthésie-Réanimation & $4,1 \%$ \\
\hline Néonatalogie & $3 \%$ \\
\hline Chirurgie & $2,9 \%$ \\
\hline Pédiatrie & $1,5 \%$ \\
\hline Cancérologie & $1,4 \%$ \\
\hline Bloc opératoire & $1,4 \%$ \\
\hline Gérontologie & $1,4 \%$ \\
\hline Psychiatrie & $1,4 \%$ \\
\hline Cardiologie & $0,6 \%$ \\
\hline Gynécologie & $0,6 \%$ \\
\hline Pneumologie & $0,4 \%$ \\
\hline Soins palliatifs & $0,3 \%$ \\
\hline Néphrologie & $0,3 \%$ \\
\hline Neurologie & $0,1 \%$ \\
\hline Odontologie & $0,1 \%$ \\
\hline Prélèvement multi-organes & $0,1 \%$ \\
\hline Urologie & $0,1 \%$ \\
\hline
\end{tabular}




\section{Activités de soins transversales}

\begin{tabular}{|l|c|}
\hline Soins infirmiers & $26,3 \%$ \\
\hline Hygiène & $3,3 \%$ \\
\hline Médicament & $3,3 \%$ \\
\hline Hémovigilance & $2,2 \%$ \\
\hline Douleur & $1,7 \%$ \\
\hline Raisonnement clinique & $1,4 \%$ \\
\hline Éducation thérapeutique & $1,1 \%$ \\
\hline Éthique & $0,1 \%$ \\
\hline
\end{tabular}

\section{Activités techniques et logistiques}

\begin{tabular}{|l|c|}
\hline Sécurité incendie & $4,1 \%$ \\
\hline Logistique et techniques & $0,5 \%$ \\
\hline
\end{tabular}

\section{Activités support}

\begin{tabular}{|l|c|}
\hline Ergonomie & $4 \%$ \\
\hline Communication & $5,9 \%$ \\
\hline Management - Ressources humaines & $1,5 \%$ \\
\hline Informatique - Logiciel & $0,7 \%$ \\
\hline Qualité & $0,7 \%$ \\
\hline
\end{tabular}

\section{$\rightarrow$ Autre}

En ce qui concerne les activités par discipline, le SAMU (CESU6 $)$ arrive nettement en tête des réponses (cela est probablement dû à l'antériorité de formations procédurales dans ce domaine). La médecine d'urgence, l'obstétrique et la néonatalogie ainsi que l'anesthésie réanimation sont également bien placées, et cette distribution correspond aux spécialités dites à risques. Parmi les activités de soins transversales, ce sont les soins infirmiers qui sont le plus souvent concernés par ces techniques (approches procédurales de soins infirmiers).

II est intéressant de noter par ailleurs que les activités support et managériales développent aussi des activités de simulation, on retrouve par exemple historiquement toutes les démarches de simulation incendie.

6. Centre d'enseignement des soins d'urgence. 


\section{Contribution de l'université au développement de la simulation dans la formation professionnelle continue (Tableau 5)}

Il est intéressant de noter que depuis en moyenne 3 à 4 ans, plusieurs diplômes universitaires à destination des professionnels de santé médicaux et paramédicaux se développent. Parmi ces diplômes, certains sont clairement dédiés à l'activité de simulation et permettent de former les formateurs (deux diplômes répertoriés comme tel). Mais beaucoup d'enseignements intègrent d'ores et déjà des méthodes de simulation comme outils pédagogiques. Quelques références sont données à titre d'exemple.

Tableau 5. DU utilisant la simulation comme outil pédagogique (liste non exhaustive)

\begin{tabular}{|c|c|}
\hline Intitulé du diplôme & Modalité de formation par la simulation \\
\hline DU Psycho-sexologie (Paris Descartes) & Simulation de consultation : 6 séances de 3 heures \\
\hline DU Français médical (Lyon 1) & Simulation d'un interrogatoire de patient \\
\hline DU Pratiques chirurgicales en cancérologie (Lyon 1) & Simulation de comités de décision thérapeutique pluridisciplinaire \\
\hline DU Prise en charge des traumatismes sévères (Lyon 1) & $\begin{array}{l}\text { Atelier de simulation de prise en charge pré-hospitalière, transport et } \\
\text { accueil et traitement hospitaliers (adulte, enfant) }\end{array}$ \\
\hline $\begin{array}{l}\text { DU Régulation médicale dans un centre de réception et } \\
\text { de régulation des appels (CRRA) du SAMU (Lyon 1) }\end{array}$ & Simulation d'appels téléphoniques à la régulation en ateliers \\
\hline $\begin{array}{l}\text { DU Gestion des risques dans la prise en charge des } \\
\text { patients en établissement de santé (UFR de médecine } \\
\text { d'Angers) }\end{array}$ & $\begin{array}{l}3 \text { modules utilisant la simulation haute-fidélité de situations cliniques } \\
\text { en anesthésie réanimation obstétrique, prise en charge } \\
\text { thérapeutique }\end{array}$ \\
\hline $\begin{array}{l}\text { DIU de perfectionnement en anesthésie pédiatrique } \\
\text { (UFR Angers, Rennes, Nantes, Tours) }\end{array}$ & $\begin{array}{l}1 \text { module : séances de simulation : } \\
\text { - simulation et anesthésie pédiatrique ; } \\
\text { - présentation du matériel ; } \\
\text { - } 4 \text { scénarios d'anesthésie pédiatrique sur mannequins haute- } \\
\text { fidélité. }\end{array}$ \\
\hline $\begin{array}{l}\text { DU Simulation pour l'apprentissage des sciences de la } \\
\text { santé (UFR de médecine et des sciences de la santé } \\
\text { de Brest) }\end{array}$ & Formation de formateur à la simulation \\
\hline $\begin{array}{l}\text { DU Formateur à l'enseignement sur simulateur, (Paris } \\
\text { Descartes) }\end{array}$ & Formation de formateur à la simulation \\
\hline
\end{tabular}

\section{Synthèse de l'enquête simplifiée}

Bien que le taux de réponse reste modéré, on constate au total que les activités de simulation existent sur le territoire national, mais la densité en est assez faible. Celles-ci se regroupent plus particulièrement autour des disciplines cliniques dites "à risques ", et s'étendent sur des champs non cliniques comme par exemple la gestion des ressources humaines à l'instar de ce qui se pratique au sein des entreprises.

Pour répondre à notre premier objectif d'évaluer à la fois le niveau de connaissance de ces méthodes et l'attrait qu'elles suscitent de la part des professionnels de santé, cette première analyse montre qu'il existe une sensibilité aux techniques de formation par la simulation. On peut aussi noter, en marge des réponses au questionnaire, que plusieurs centres ont exprimé par contact direct leur grand intérêt pour le sujet et le souhait de recevoir les résultats de l'étude.

Compte tenu de la répartition des réponses, il semble probable que la mutualisation des ressources autour de plates-formes pourraient sans doute permettre à plus d'établissements d'accéder à ce type d'activité. 


\subsubsection{Résultat du questionnaire d'enquête complet auprès des centres de simulation français constitués ou en projet}

Répartition géographique des centres de simulation (structures formalisées utilisant des méthodes « haute-fidélité »)

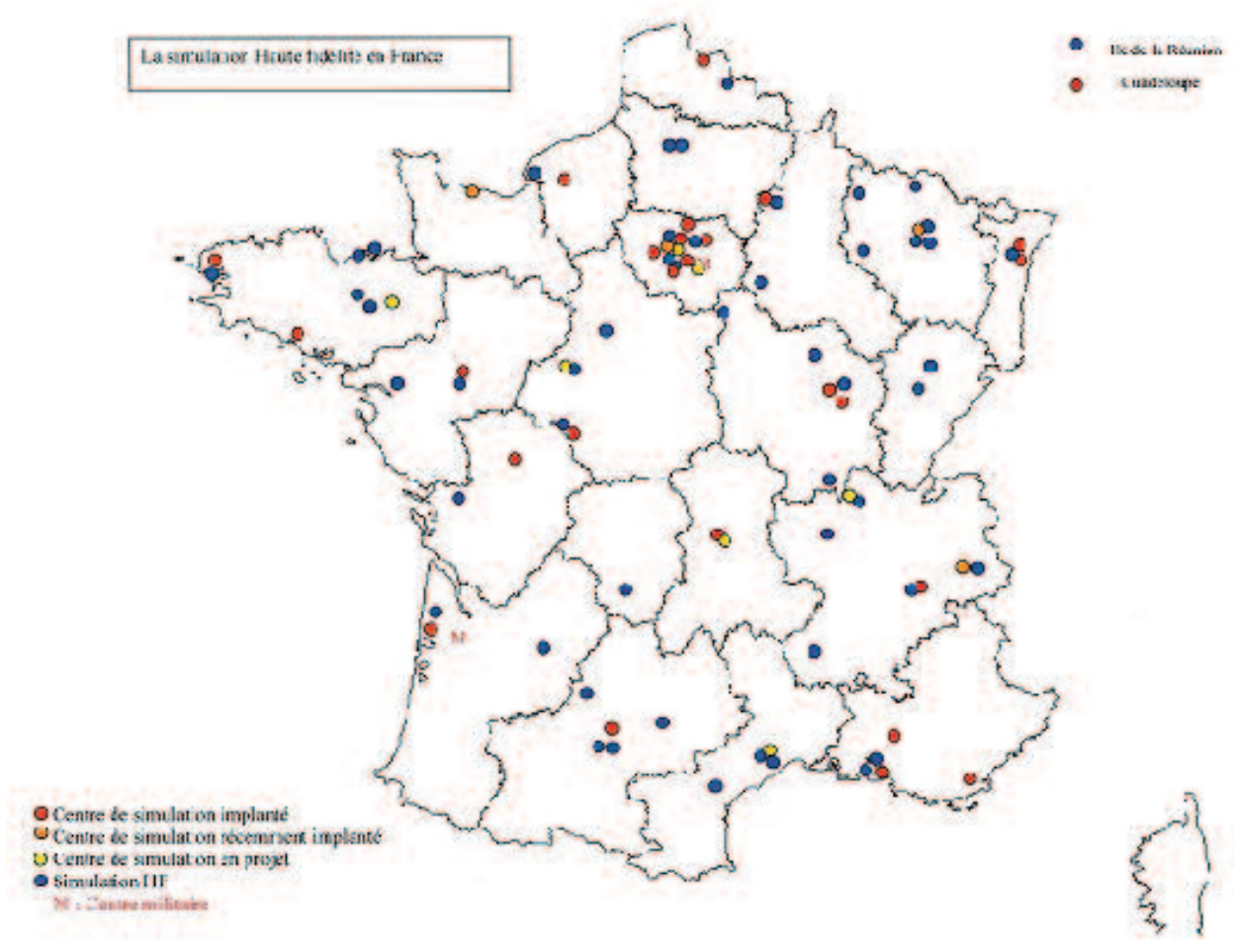

Figure 4. Implantation des centres de simulation en France

\section{Structure}

L'ensemble répertorié à ce jour des centres de simulation français, trente-quatre au total, a été sollicité sur la base des informations délivrées par l'AFSARMU. Vingt et un centres ont répondu, soit un taux de réponse de 63,6\%. Parmi les non-répondants, deux centres sont en projet.

\section{$\rightarrow$ Liste des établissements répondants}

- Assistance publique - Hôpitaux de Marseille ;

- BSPP - Paris ;

- CESIMS - Centre d'enseignement par la simulation pour les métiers de la santé - Dijon ;

- CHU Angers ;

- CHU Charles-Nicolle - Rouen ;

- CHU Clermont-Ferrand ;

- Centre d'instruction aux techniques de réanimation de l'avant (école du Val-de-Grâce) - Paris ;

- Centre de simulation en santé - Brest ; 
- ENSOSP - Aix-en-Provence ;

- FORRSIM - Faculté de médecine - Nice ;

- Groupe Infinite Medical Education (GIME) - Paris ;

- Hôpital Joseph-Ducuing - Toulouse ;

- Hôtel-Dieu, Cochin ;

- ILUMENS - Paris ;

- IRCAD - Strasbourg ;

- Laboratoire d'enseignement par simulation de Savoie, centre d'enseignement des soins d'urgence de Savoie - Centre hospitalier de Chambéry ;

- Laboratoire de simulation universitaire - Normandie-Caen - (LabSUN) ;

- UFR médecine - Reims ;

- UP2F - Darois - (Côte-d'Or) ;

- Université de Strasbourg.

\section{Date de création, statut juridique et rattachement}

\begin{tabular}{|c|c|c|c|}
\hline \multicolumn{3}{|c|}{ Taux de réponse : $90,5 \%$} & \multirow{3}{*}{$5,3 \%$} \\
\hline & $\mathrm{Nb}$ & $\%$ cit. & \\
\hline 1977 & 1 & $5,3 \%$ & \\
\hline 1992 & 1 & $5,3 \%$ & $5,3 \%$ \\
\hline 2002 & 1 & $5,3 \%$ & $5,3 \%$ \\
\hline 2005 & 2 & $10,5 \%$ & $10,5 \%$ \\
\hline 2006 & 1 & $5,3 \%$ & $5,3 \%$ \\
\hline 2007 & 1 & $5,3 \%$ & $5,3 \%$ \\
\hline 2008 & 2 & $10,5 \%$ & $10,5 \%$ \\
\hline 2009 & 2 & $10,5 \%$ & $10,5 \%$ \\
\hline 2010 & 5 & $26,3 \%$ & $26,3 \%$ \\
\hline 2011 & 3 & $15,8 \%$ & $15,8 \%$ \\
\hline Total & 19 & $100,0 \%$ & \\
\hline
\end{tabular}

$\rightarrow$ Les centres publics (hormis les centres des armées) sont majoritairement rattachés et à part égale aux universités ou aux CHU. Les centres privés sont constitués en centres de formation indépendants.

$\rightarrow$ Plus de la moitié des centres a été créée après 2008, majoritairement avec un statut public. On retrouve néanmoins deux centres des armées, dont le corps des sapeurs pompiers de Paris, quatre centres privés, dont un centre ESPIC (ex : PSPH).

\begin{tabular}{|c|c|c|c|}
\hline \multicolumn{3}{|l|}{ Taux de réponse : $\mathbf{9 5 , 2} \%$} & \multirow{3}{*}{$30,0 \%$} \\
\hline & $\mathrm{Nb}$ & $\%$ cit. & \\
\hline $\mathrm{CHU}$ & 6 & $30,0 \%$ & \\
\hline Université & 6 & $30,0 \%$ & $30,0 \%$ \\
\hline Armée & 3 & $15,0 \%$ & $15,0 \%$ \\
\hline Organisme de formation indépendant & 2 & $10,0 \%$ & $10,0 \%$ \\
\hline Association indépendante & 2 & $10,0 \%$ & $10,0 \%$ \\
\hline $\mathrm{CH}$ & 1 & $5,0 \%$ & $5,0 \%$ \\
\hline Total & 20 & $100,0 \%$ & \\
\hline
\end{tabular}




\section{Management et gouvernance}

Tous les centres sont dirigés par des médecins sauf trois qui le sont par des managers (dont deux centres privés). Dans près de $50 \%$ des cas, il s'agit d'un professeur des universités (PU-PH).

Les disciplines, majoritairement représentées, sont l'anesthésie-réanimation (sept centres), la médecine d'urgence (trois centres) et la chirurgie (un centre).

\section{$\Rightarrow$ Équivalents temps plein (ETP) pour la direction du centre}

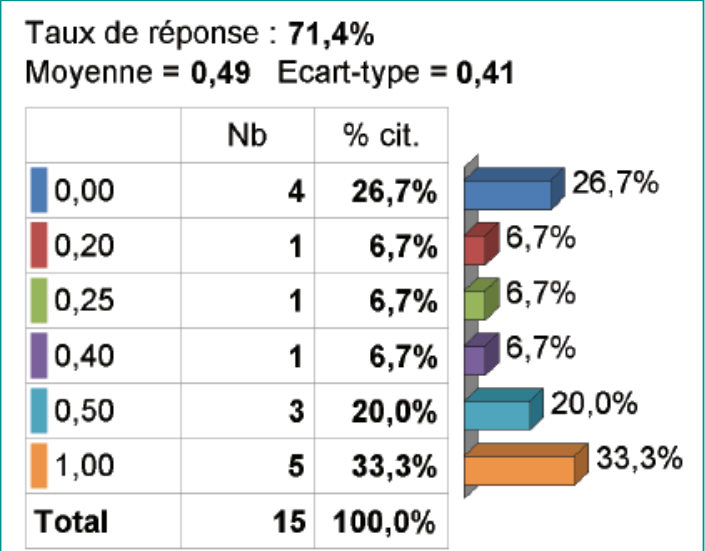

\author{
$\rightarrow$ Le temps n'est pas toujours dédié à la fonction de \\ responsable. \\ $\rightarrow$ Cinq centres seulement y consacrent un temps \\ plein.
}

\section{Formalisation de la gouvernance}

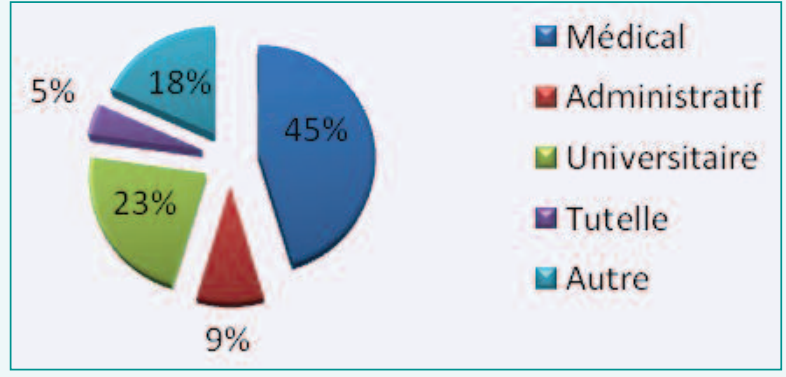

$\rightarrow$ La gouvernance est formalisée pour la moitié des centres. Dans la majorité des cas, elle repose sur les composantes hospitalo-universitaires.

\section{Ressources humaines}

\begin{tabular}{|c|c|c|c|}
\hline \multicolumn{3}{|c|}{ Taux de réponse : $\mathbf{1 0 0 , 0 \%}$} & \multirow[b]{3}{*}{$66,7 \%$} \\
\hline & $\mathrm{Nb}$ & $\%$ cit. & \\
\hline Public & 14 & $66,7 \%$ & \\
\hline Privé & 3 & $14,3 \%$ & $14,3 \%$ \\
\hline Militaire & 2 & $9,5 \%$ & $9,5 \%$ \\
\hline Associatif & 1 & $4,8 \%$ & $4,8 \%$ \\
\hline $\mathrm{PSPH}$ & 1 & $4,8 \%$ & $4,8 \%$ \\
\hline GIP GCS & 0 & $0,0 \%$ & $0,0 \%$ \\
\hline Total & 21 & $100,0 \%$ & \\
\hline
\end{tabular}

$\rightarrow$ Les établissements n'ont pas systématiquement répondu à toutes les questions. Quinze établissements sur vingt et un ont répondu et, parmi ceux-ci, toutes les questions n'ont pas été systématiquement renseignées. Compte tenu de sa nature très particulière, un centre* se distingue notablement des autres établissements. II a donc été volontairement retiré de l'échantillon et les résultats sont présentés à part dans le tableau ci-dessous. Cela permet d'obtenir une vision plus objective de la moyenne des autres centres. 


\section{$\rightarrow$ Ressources humaines en ETP (équivalent temps plein)}

Les établissements disposent en moyenne de 0,36 ETP de personnel de formation vraiment dédié. Les établissements ont cependant du mal à identifier les ressources dédiées en personnel (parfois confondues avec celles d'autres activités) En dehors des formateurs, peu d'autres catégories de personnel sont dédiées : en moyenne 0,48 ETP et majoritairement des secrétaires et des personnels techniques.

Tableau 6. Ressources humaines en ETP

\begin{tabular}{|c|c|c|c|}
\hline ETP & Moyenne ETP* & $\begin{array}{l}\text { Nbre de centres } \\
\text { répondants* }\end{array}$ & $\begin{array}{c}\text { Résultats pour } \\
1 \text { centre }\end{array}$ \\
\hline Formateurs permanents & 0,36 & 14 & 8 \\
\hline Formateurs ponctuels & 0,44 & 6 & 10 \\
\hline Encadrement & 0,34 & 7 & 0,70 \\
\hline Hôtesse & 0 & 0 & 4 \\
\hline Secrétaire & 0,50 & 9 & 3 \\
\hline Entretien des locaux & 0,13 & 3 & 2 \\
\hline Maintenance des équipements & 0,53 & 5 & 9 \\
\hline Communication & 0 & 0 & 10 \\
\hline Démarchage & 0,50 & 1 & 1 \\
\hline Qualiticien & 0,50 & 1 & 0 \\
\hline Autre personnel technique & 0,75 & 2 & 15 \\
\hline Autre personnel administratif & 0 & 1 & 11 \\
\hline
\end{tabular}

*Moyenne sur les centres répondants ;

${ }^{* *}$ Centre sorti de l'échantillon.

\section{Formateurs}

La majorité des formateurs est représentée par des praticiens hospitaliers et des infirmiers, qu'il s'agisse de formateurs ponctuels ou permanents.

Les formateurs permanents suivent davantage les formations à la simulation. Parmi les personnes formées (formateurs permanents et formateurs ponctuels), environ la moitié suit des formations diplômantes.

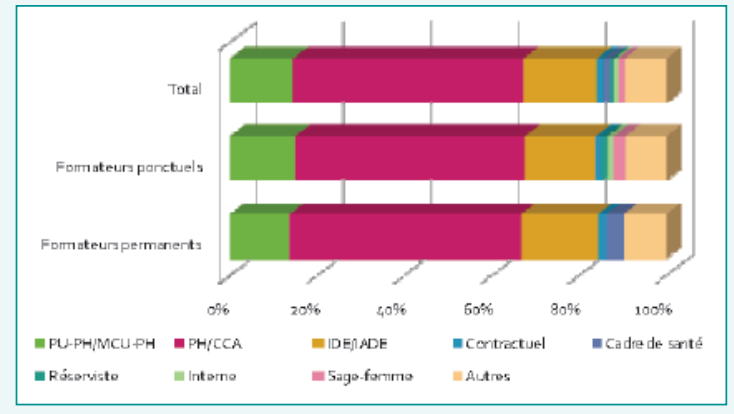

Répartition des formateurs

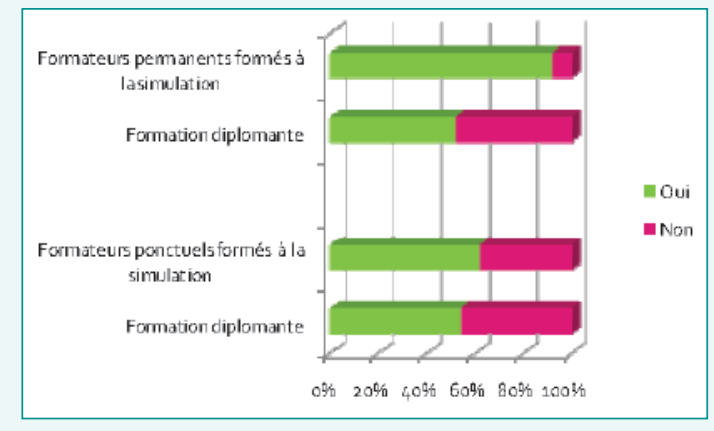

Formation des formateurs 
Au total, $78 \%$ des centres disposent de locaux dédiés. En moyenne, ils disposent de 2,9 salles de simulation, et $44 \%$ d'entre eux disposent d'au moins 3 salles. Sur 15 salles identifiées, $73 \%$ disposent d'équipement vidéo. Chaque centre dispose d'au moins une salle de contrôle. Sur seize centres, quinze disposent d'au moins une salle de debriefing (un centre dispose de deux salles, et un centre dispose de cinq salles).

L'affectation des salles est très diversifiée sur l'ensemble des disciplines enseignées en simulation.

\section{Locaux affectés aux séances de formation}

\begin{tabular}{|c|c|c|c|}
\hline \multicolumn{3}{|c|}{ Taux de réponse : $\mathbf{8 5 , 7 \%}$} & \multirow[b]{3}{*}{$77,8 \%$} \\
\hline & $\mathrm{Nb}$ & $\%$ cit. & \\
\hline Oui & 14 & $77,8 \%$ & \\
\hline Non & 4 & $22,2 \%$ & $22,2 \%$ \\
\hline Total & 18 & $100,0 \%$ & \\
\hline
\end{tabular}

Locaux dédiés exclusivement à la simulation

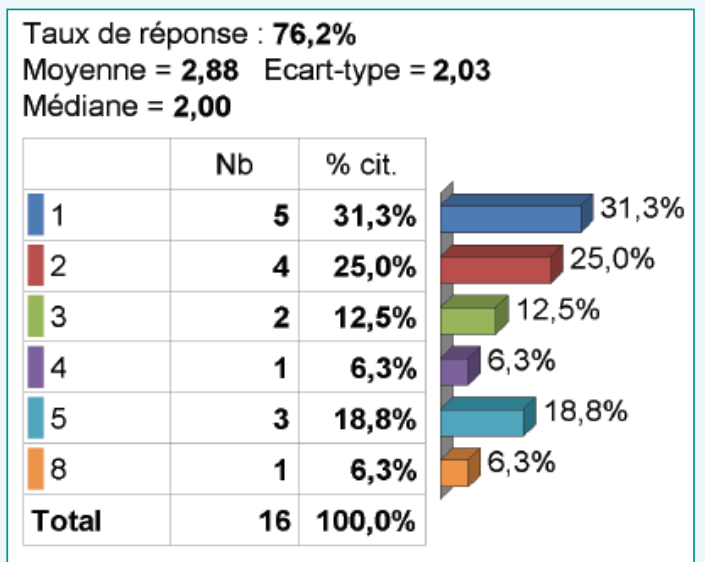

\section{Affectation des salles de simulation}

\begin{tabular}{|l|l|}
\hline Anesthésie Réanimation - Urgences & 5 \\
\hline Salle de réveil et réanimation & 1 \\
\hline Chambre de réanimation & 1 \\
\hline Médecine d'urgence & 2 \\
\hline Prise en charge de I'urgence extra-hospitalière & 1 \\
\hline AR-MU-Pedia-Néonat & 1 \\
\hline Multi-disciplinaire & 3 \\
\hline Salle de naissance & 2 \\
\hline Bloc opératoire & 5 \\
\hline Chirurgie mini-invasive et robotisée & 1 \\
\hline Salle de radiologie interventionnelle et endoscopie & 1 \\
\hline 3 salles avec le même équipement matériel médico-chirurgical & 1 \\
\hline Chambre conventionnelle & 1 \\
\hline Bureau de consultation & 1 \\
\hline Adulte & 1 \\
\hline Sédation et geste d'urgence en dentaire & 1 \\
\hline
\end{tabular}




\section{$\rightarrow$ Autres locaux}

Sept centres (sur onze répondants) disposent de vestiaires, environ onze centres disposent d'au moins un bureau pour les formateurs (sur quatorze répondants) et seul un centre sur quinze dispose d'une salle de stockage dédiée (sur seize répondants). La plupart des centres semblent mal équipés pour tous les locaux annexes et particulièrement pour ceux dédiés à la convivialité.

\section{Équipement des centres de simulation en matériel haute-fidélité (Tableau 7)}

Un constat s'impose, les centres de simulation sont globalement peu équipés. Le matériel de réalité virtuelle est inexistant dans les centres publics.

Tableau 7. Équipement

\begin{tabular}{|c|c|c|c|}
\hline & $\begin{array}{l}\text { Nbre de centres } \\
\text { répondants }\end{array}$ & Nombre d'équipement & $\begin{array}{l}\text { Moyenne } \\
\text { par centre }\end{array}$ \\
\hline Mannequin adulte & 17 & $\begin{array}{l}\text { - } 4 \text { centres disposent de } 3 \text { mannequins } \\
\text { - } 3 \text { centres disposent de } 2 \text { mannequins } \\
\text { - } 10 \text { centres disposent d'un mannequin }\end{array}$ & 1,6 \\
\hline Mannequin enfant & 9 & - 1 mannequin par centre & 1 \\
\hline Mannequin nouveau-né & 10 & $\begin{array}{l}\text { - } 8 \text { centres disposent de } 1 \text { mannequin } \\
\text { - } 2 \text { centres disposent de } 2 \text { mannequins }\end{array}$ & 1,2 \\
\hline Simulateur chirurgical et la paroscopie & 2 & $\begin{array}{l}\text { - } 6 \text { simulateurs pour ce centre (3FLS, } \\
1 \text { SIMBIOTICS et } 2 \text { SIMSURGERY } \\
\text { - } 2 \text { FLS pour un centre }\end{array}$ & 4 \\
\hline Réalité virtuelle & 1 & - 1 digital trainer & 1 \\
\hline
\end{tabular}

\section{Financement}

\begin{tabular}{|c|c|c|}
\hline \multicolumn{3}{|c|}{$\begin{array}{l}\text { Taux de réponse : } \mathbf{5 7 , 1} \% \\
\text { Moyenne }=\mathbf{2 5 5} 541,67 \quad \text { Ecart-type }=\mathbf{4 6 4 5 3 2 , 1 4} \\
\text { Médiane }=\mathbf{7 6} \mathbf{2 0 0}, 00\end{array}$} \\
\hline & $\mathrm{Nb}$ & $\%$ cit. \\
\hline Moins de 50000 & 3 & $25,0 \%$ \\
\hline De 50000 à 99999 & 4 & $33,3 \%$ \\
\hline De 100000 à 499999 & 3 & $25,0 \%$ \\
\hline De 500000 à 999999 & 1 & $8,3 \%$ \\
\hline 1000000 et plus & 1 & $8,3 \%$ \\
\hline Total & 12 & $100,0 \%$ \\
\hline
\end{tabular}

Répartition des financements de départ $\rightarrow$ Tous les centres considèrent avoir eu des difficultés, voire de grandes difficultés, pour obtenir le financement. Les délais d'obtention du financement varient entre une semaine et trois ans.

$\rightarrow$ Plus de la moitié des établissements a démarré avec un financement initial de moins de 100000 euros (remarque : un mannequin haute-fidélité adulte coûte en moyenne 40000 euros).

L'origine des financements initiaux semble relever généralement de l'université et de fonds publics régionaux et, pour un centre, de fonds privés. Les résultats sont présentés avec moyenne et médiane pour minimiser l'impact des valeurs extrêmes dans l'interprétation des données.

II a été difficile d'obtenir des renseignements précis sur les éléments budgétaires qui parfois relèvent de données confidentielles. Le financement annuel (cinq centres) varie entre 5000 et 12000000 d'euros.

Il est par ailleurs difficile de savoir si les charges de personnels sont toujours incluses dans les budgets annoncés. 
Quatre centres sur six déclarent être en déficit de financement annuel.

Il est difficile d'évaluer les tarifs individuels de formation sur cinq réponses. Le tarif à la demijournée varie entre 15 et 750 euros. Les tarifs de groupe varient entre 100 et 10000 euros par séance (sept centres répondants). Certains centres proposent des formations gratuites ou des formations payantes.

\section{- Pédagogie}

\section{$\rightarrow$ Offre de formation}

Quinze centres sur dix-sept ont un projet pédagogique, et pour près de $50 \%$, celui-ci est formalisé.

Les centres contractualisent peu avec d'autres structures de formation, environ la moitié. Quand la contractualisation existe, elle est réalisée avec les réseaux, les IFSI et IFCS, des organismes de santé où les facultés de pharmacie.

\begin{tabular}{|l|c|}
\hline & Montants (en euros) \\
\hline Actionnaire & 100 \\
\hline Association & 100 \\
\hline Autres & 2400 \\
\hline Pompiers & 10000 \\
\hline ARH & 20000 \\
\hline Banque assurance & 40000 \\
\hline Conseil régional & 40000 \\
\hline Centre hospitalier & 100000 \\
\hline Faculté de médecine & 200000 \\
\hline Université & 32000 \\
\hline Conseil général & 540000 \\
\hline United States Surgical Corporation & 1500000 \\
\hline
\end{tabular}

Répartition des contributeurs au financement

L'activité de formation se répartit en deux groupes : les centres qui dispensent moins de 40 heures par an (trois centres) et ceux qui dispensent plus de 150 heures (trois centres).

\section{Répartition de la population formée}

Treize centres sur dix-huit proposent de la formation initiale essentiellement pour des internes et des étudiants en médecine. Quatorze centres sur dix-sept proposent de la formation continue, majoritairement des médecins sénior et des paramédicaux (IDE).

\begin{tabular}{|c|c|c|c|}
\hline \multicolumn{3}{|l|}{ Taux de réponse : $61,9 \%$} & \multirow{3}{*}{$47,6 \%$} \\
\hline & $\mathrm{Nb}$ & $\%$ obs. & \\
\hline Interne médecine & 10 & $47,6 \%$ & \\
\hline Interne pharmacie & 0 & $0,0 \%$ & $0,0 \%$ \\
\hline Externe médecine & 7 & $33,3 \%$ & $33,3 \%$ \\
\hline Externe pharmacie & 0 & $0,0 \%$ & $0,0 \%$ \\
\hline Eleves Sage-femme & 2 & $9,5 \%$ & $9,5 \%$ \\
\hline Elève IDE (IADE, IBODE, Puéricultrice) & 9 & $42,9 \%$ & $42,9 \%$ \\
\hline Autres élèves paramédicaux & 3 & $14,3 \%$ & $14,3 \%$ \\
\hline Autres stagiaires & 4 & $19,0 \%$ & $19,0 \%$ \\
\hline Total & 21 & & \\
\hline
\end{tabular}

Formation initiale

\begin{tabular}{|c|c|c|c|}
\hline \multicolumn{3}{|l|}{ Taux de réponse : $61,9 \%$} & \multirow{3}{*}{$61,9 \%$} \\
\hline & $\mathrm{Nb}$ & $\%$ obs. & \\
\hline Médecin séniors & 13 & $61,9 \%$ & \\
\hline IDE (IADE & 10 & $47,6 \%$ & $47,6 \%$ \\
\hline Autre paramédicaux & 6 & $28,6 \%$ & $28,6 \%$ \\
\hline Autres professionnels (Pompiers...) & 5 & $23,8 \%$ & $23,8 \%$ \\
\hline IBODE & 5 & $23,8 \%$ & $23,8 \%$ \\
\hline Sage Femme & 5 & $23,8 \%$ & $23,8 \%$ \\
\hline Puéricultrice...) & 4 & $19,0 \%$ & $19,0 \%$ \\
\hline Pharmaciens seniors & 3 & $14,3 \%$ & $14,3 \%$ \\
\hline Total & 21 & & \\
\hline
\end{tabular}

Formation continue 


\section{$\rightarrow$ Scénarios}

Seize établissements sur dix-sept disposent de scénarios, mais leur taux de renouvellement est assez aléatoire allant de plusieurs fois par an pour cinq centres, tous les ans pour trois centres et de temps en temps pour cinq centres.

\section{Mode de progression de l'offre de formation}

II n'y a pas de stratégie formelle de renouvellement des scénarios, pas plus que de véritable stratégie pour faire progresser l'offre.

\section{Modalités de progression de l'offre de formation}

Là également, aucune stratégie particulière ne semble émerger.

\begin{tabular}{|c|c|c|c|}
\hline \multicolumn{3}{|l|}{ Taux de réponse : $\mathbf{8 1 , 0 \%}$} & \multirow{3}{*}{$20,6 \%$} \\
\hline & $\mathrm{Nb}$ & $\%$ cit & \\
\hline en fonction desdemandes qui voussontadressées & 13 & $20,6 \%$ & \\
\hline en fonction desmoyens dontvous disposez & 12 & $19,0 \%$ & $19,0 \%$ \\
\hline en saisissant des opportunités & 11 & $17,5 \%$ & $17,5 \%$ \\
\hline en recherchantles alliances avec d'autres disciplines & 9 & $14,3 \%$ & $14,3 \%$ \\
\hline en planifianteten anticipant celle-ci sur le $œ$ urt moyen ou long terme & 8 & $12,7 \%$ & $12,7 \%$ \\
\hline en réalisantuneévaluation prospective du marché & 6 & $9,5 \%$ & $9,5 \%$ \\
\hline en répondant àune contrainte forte (injonction de la structure dontvous dépendez) & 4 & $6,3 \%$ & ⿹6,3\% \\
\hline Total & 63 & $100,0 \%$ & \\
\hline
\end{tabular}

Stratégie de progression de l'offre

\section{$\rightarrow$ Évaluation de la formation}

Enfin, les formations font toujours l'objet d'une évaluation, mais par simple enquête de satisfaction. Dans très peu de cas, des évaluations écrites sont remises avec conseil sur le mode de progression.

\begin{tabular}{|c|c|c|c|}
\hline \multicolumn{3}{|l|}{ Taux de réponse : $81,0 \%$} & \multirow[b]{3}{*}{$71,4 \%$} \\
\hline & $\mathrm{Nb}$ & $\%$ obs. & \\
\hline Améliora ton des compétencos & 15 & $71,4 \%$ & \\
\hline Améliora ton des connaissanoes & 15 & $71,4 \%$ & $71,4 \%$ \\
\hline Gestion des ri sques: asso ciés auxsoins & 13 & $61,9 \%$ & $7^{11,9 \%}$ \\
\hline Etude des facteurshuma ins & 12 & $57,1 \%$ & 57 \\
\hline Si mulation de crise & 12 & $57.1 \%$ & $757,1 \%$ \\
\hline Acquisition de compé tences & 12 & $57,1 \%$ & $757,1 \%$ \\
\hline Acquisition de conna issa nces & 9 & $42,9 \%$ & $42,9 \%$ \\
\hline Reconsttution d'a ccidents & 8 & $38,1 \%$ & 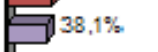 \\
\hline Dével op pement tpro fessionnel continu & 7 & $33,3 \%$ & $3: 3,3 \%$ \\
\hline EPP & 6 & $28,6 \%$ & $28,6 \%$ \\
\hline Remise à ni veau & 5 & $23,8 \%$ & $23,8 \%$ \\
\hline Habilita fon de pro essionnels & 2 & $9,5 \%$ & $9,5 \%$ \\
\hline Qualification des professiomes & 1 & $4,8 \%$ & $4,8 \%$ \\
\hline Total & 21 & & \\
\hline
\end{tabular}

Évaluation des séances

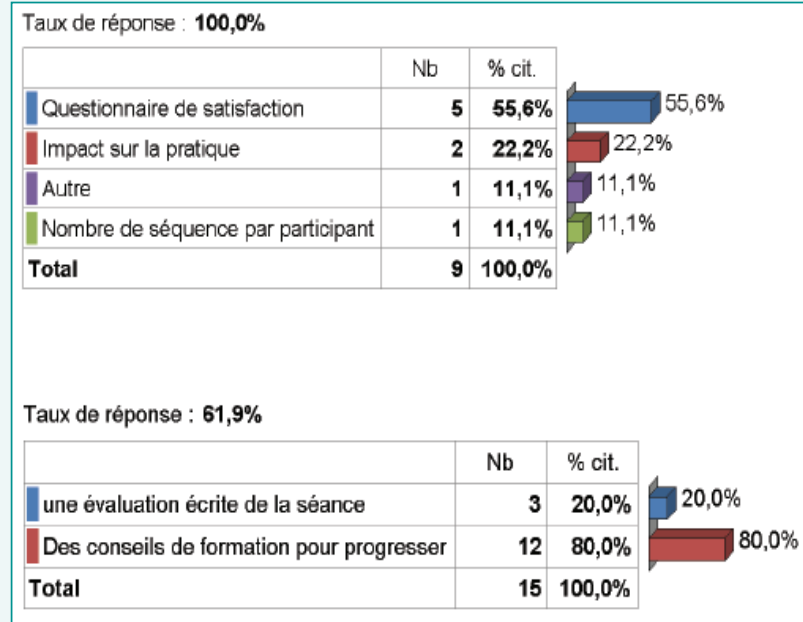

Type d'évaluation 


\section{Communication et notoriété}

$\rightarrow$ Politique de communication

Taux de réponse : $\mathbf{5 7 , 1 \%}$
\begin{tabular}{|l|r|r|}
\hline & Nb & $\%$ cit. \\
\hline Site internet & $\mathbf{1 1}$ & $\mathbf{3 5 , 5 \%}$ \\
\hline plaquete & $\mathbf{8}$ & $\mathbf{2 5 , 8 \%}$ \\
\hline rése aud de diffusion de l'informaion & $\mathbf{8}$ & $\mathbf{2 5 , 8 \%}$ \\
\hline autre & $\mathbf{4}$ & $\mathbf{1 2 , 9 \%}$ \\
\hline Total & $\mathbf{3 1}$ & $\mathbf{1 0 0 , 0}$ \\
\hline
\end{tabular}

Outils de communication $\rightarrow$ Sur dix-sept centres, un peu plus des $2 / 3$, disposent d'une politique de communication. Les outils de communication se répartissent en site internet, plaquette, réseau de diffusion.

\section{Progression de l'activité sur 3 ans}

Taux de réponse : $\mathbf{4 7 , 6 \%}$
\begin{tabular}{||r|r|r|}
\hline & $\mathrm{Nb}$ & $\%$ cit. \\
\hline Forte progression & $\mathbf{7}$ & $\mathbf{7 0 , 0}$ \\
\hline Progression & $\mathbf{2}$ & $\mathbf{2 0 , 0 \%}$ \\
\hline Stagnante & $\mathbf{0}$ & $\mathbf{0 , 0} \%$ \\
\hline Baisse & $\mathbf{1}$ & $\mathbf{1 0 , 0 \%}$ \\
\hline Forte baisse & $\mathbf{0}$ & $\mathbf{0 , 0} \%$ \\
\hline Total & $\mathbf{1 0}$ & $\mathbf{1 0 0 , 0 \%}$ \\
\hline $10,0 \%$ \\
$0,0 \%$ \\
\hline
\end{tabular}

$\rightarrow$ Le taux de progression des centres est plutôt positif, voire très positif.

\section{$\rightarrow$ Notoriété}

L'attractivité est essentiellement locale et régionale.

Le rayonnement est à peu près superposable, sauf pour deux centres qui ont des liens avec des universités étrangères.

Taux de réponse : $\mathbf{7 6 , 2} \%$
\begin{tabular}{||r|r|r|}
\hline & $\mathrm{Nb}$ & $\%$ cit \\
\hline \hline Local & $\mathbf{4}$ & $\mathbf{1 9 , 0} \%$ \\
\hline \hline Départemental & $\mathbf{2}$ & $\mathbf{9 , 5 \%}$ \\
\hline \hline Régional & $\mathbf{6}$ & $\mathbf{2 8 , 6 \%}$ \\
\hline \hline Interrégional & $\mathbf{2}$ & $\mathbf{9 , 5 \%}$ \\
\hline \hline National & $\mathbf{2}$ & $\mathbf{9 , 5 \%}$ \\
\hline \hline International & $\mathbf{5}$ & $\mathbf{2 3 , 8 \%}$ \\
\hline Total & $\mathbf{2 1}$ & $\mathbf{1 0 0 , 0 \%}$ \\
\hline
\end{tabular}

Attractivité

\begin{tabular}{|c|c|c|c|}
\hline \multicolumn{3}{|l|}{ Taux de réponse : $76,2 \%$} & \multirow{3}{*}{$25,0 \%$} \\
\hline & $\mathrm{Nb}$ & $\%$ cit. & \\
\hline Pasconnu & 5 & $25,0 \%$ & \\
\hline connu dans ma ville & 3 & $15,0 \%$ & $15,0 \%$ \\
\hline Rayonnementrégionale & 2 & $10,0 \%$ & ]10,0\% \\
\hline Rayonnementinter régional & 1 & $5,0 \%$ & $5,0 \%$ \\
\hline Rayonnementnational & 4 & $20,0 \%$ & $120,0 \%$ \\
\hline Rayonnementinternational & 5 & $25,0 \%$ & $25,0 \%$ \\
\hline Total & 20 & $100,0 \%$ & \\
\hline
\end{tabular}

Notoriété 


\section{Recherche}

\begin{tabular}{|c|c|c|c|}
\hline \multicolumn{3}{|l|}{ Taux de réponse : $\mathbf{5 5 , 6 \%}$} & \multirow{3}{*}{$25,9 \%$} \\
\hline & $\mathrm{Nb}$ & $\%$ cit. & \\
\hline Programme en cours & 7 & $25,9 \%$ & \\
\hline Axes de recherche & 7 & $25,9 \%$ & $25,9 \%$ \\
\hline Laboratoire de recherche & 7 & $25,9 \%$ & $25,9 \%$ \\
\hline Publication (joindre publication) & 4 & $14,8 \%$ & $14,8 \%$ \\
\hline Financement & 2 & $7,4 \%$ & $7,4 \%$ \\
\hline Total & 27 & $100,0 \%$ & \\
\hline
\end{tabular}

$\rightarrow$ Treize centres sur dix-sept déclarent avoir une activité de recherche. Peu de centres disposent d'un financement spécifique pour réaliser leur activité de recherche.

\subsubsection{Synthèse de l'état des lieux de la simulation en France}

L'activité de simulation en santé, même si elle n'est pas encore très dense, se répartit sur l'ensemble du territoire national et intéresse toutes les disciplines. Beaucoup d'activités se « rapprochant » de la simulation existent, mais demeurent assez artisanales.

Les centres de simulation français disposent en général de locaux dédiés, mais la surface en reste assez modeste (en moyenne moins de 3 salles par centre équipées ou non de systèmes vidéo). Les locaux annexes permettant convivialité et debriefing sont peu développés. Tous les centres déclarent utiliser au moins un matériel haute-fidélité. Cependant, de manière générale, les matériels et équipements sont peu nombreux et encore assez peu diversifiés à l'exception de rares établissements (en moyenne 1,6 mannequin HF adulte et 1 mannequin HF enfant). Par exemple, les simulateurs chirurgicaux sont rarement cités au sein des centres constitués. La simulation ayant recours à des environnements virtuels est quasi inexistante.

Beaucoup de thèmes ou de disciplines sont couverts par la simulation même si certains thèmes sont plus récurrents tels que ceux se rapportant à l'anesthésie réanimation, à la médecine d'urgence et à la périnatalité (néonatalogie et obstétrique), de même que tout ce qui concerne les soins infirmiers.

Les ressources humaines allouées sont faibles et particulièrement pour le personnel support (technicien, hôtesse et secrétaire). En revanche, il existe une réelle compétence au sein des centres puisqu'ils disposent le plus souvent de formateurs formés à la simulation (formations diplômantes).

La qualité des réponses concernant les ressources humaines montre une difficulté à évaluer précisément le niveau de celles-ci, souvent intriquées avec les personnels des services de soins des établissements. Il est probable qu'une part non négligeable de l'activité soit réalisée sur du temps non rémunéré (temps personnel, repos de garde, etc.). De la même manière que pour les ressources humaines, les structures semblent mal définies, les organigrammes et la gouvernance sont peu ou pas formalisés. Les structures présentent une grande disparité en matière d'organisation et de modalités de formation dispensées. II est par ailleurs difficile d'évaluer le niveau d'activité moyen des centres ; sur les six centres qui ont répondu, on identifie deux groupes : ceux dont l'activité est régulière et opérationnelle (> $150 \mathrm{~h} / \mathrm{mois}$ ) et ceux dont l'activité semble plus aléatoire (<40 h/mois).

Les centres souffrent d'un manque de règles ou de bonnes pratiques (par exemple les résultats de la séance font rarement l'objet d'une fiche d'aide à la progression), d'une grande dispersion des méthodes, des moyens, et des tarifs de formation. 
Le financement est assez pauvre et de sources hétérogènes, les centres ont en général rencontré beaucoup de difficultés à réunir le financement de départ et à assurer le financement du fonctionnement. Très peu sont autofinancés par les prestations proposées. II existe une activité de recherche, mais elle est peu développée et les sources de financement mal définies.

Sur l'ensemble des réponses, deux centres se distinguent nettement des autres (un centre rattaché à l'université et un centre privé) en termes de moyens financiers, de ressources humaines et de programmes de formation. Ils ne sont pas représentatifs de la réalité actuelle sur le territoire national.

Bilan national actuel de la simulation en santé : peut beaucoup mieux faire...

$\rightarrow$ Une activité de faible densité, mais répartie sur l'ensemble du territoire.

$\rightarrow$ Des moyens financiers faibles et d'origines éparses.

$\rightarrow$ Un équipement pauvre, des outils HF disséminés et la plupart du temps peu utilisés.

$\rightarrow$ Des formateurs compétents, mais dont le temps dédié est difficile à évaluer.

$\rightarrow$ Une structuration en devenir et très hétérogène.

$\rightarrow$ Un déficit notable en bonnes pratiques et en méthodes validées.

$\rightarrow$ Un certain niveau « d'artisanat».

$\rightarrow$ L'absence de recherche structurée. 


\section{Simulation en santé : techniques, bonnes pratiques et perspectives}

\subsection{Techniques de simulation}

II importe de décrire les différentes techniques de simulation le plus généralement utilisées dans le domaine de la santé $(67,79)$.

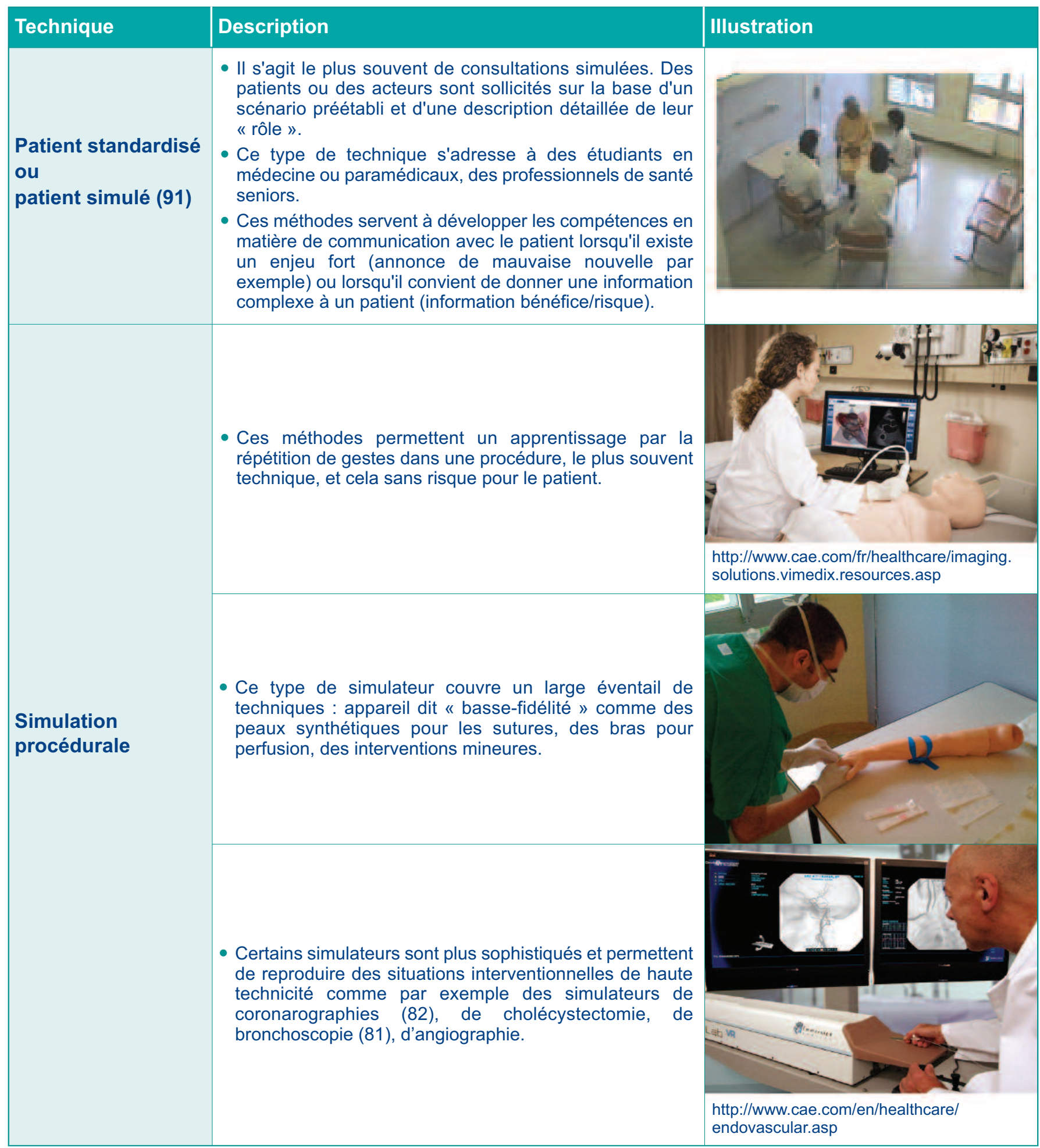




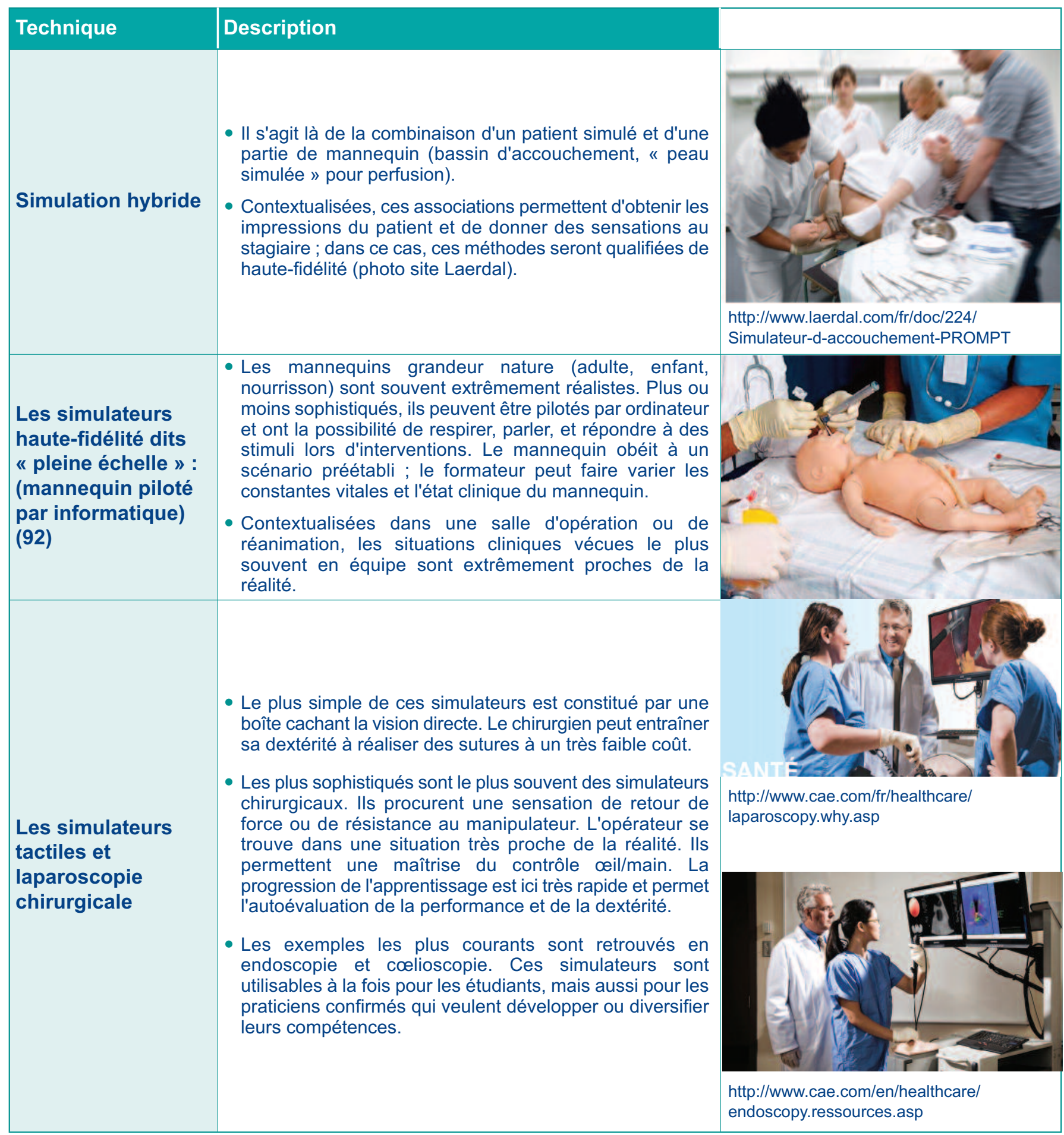




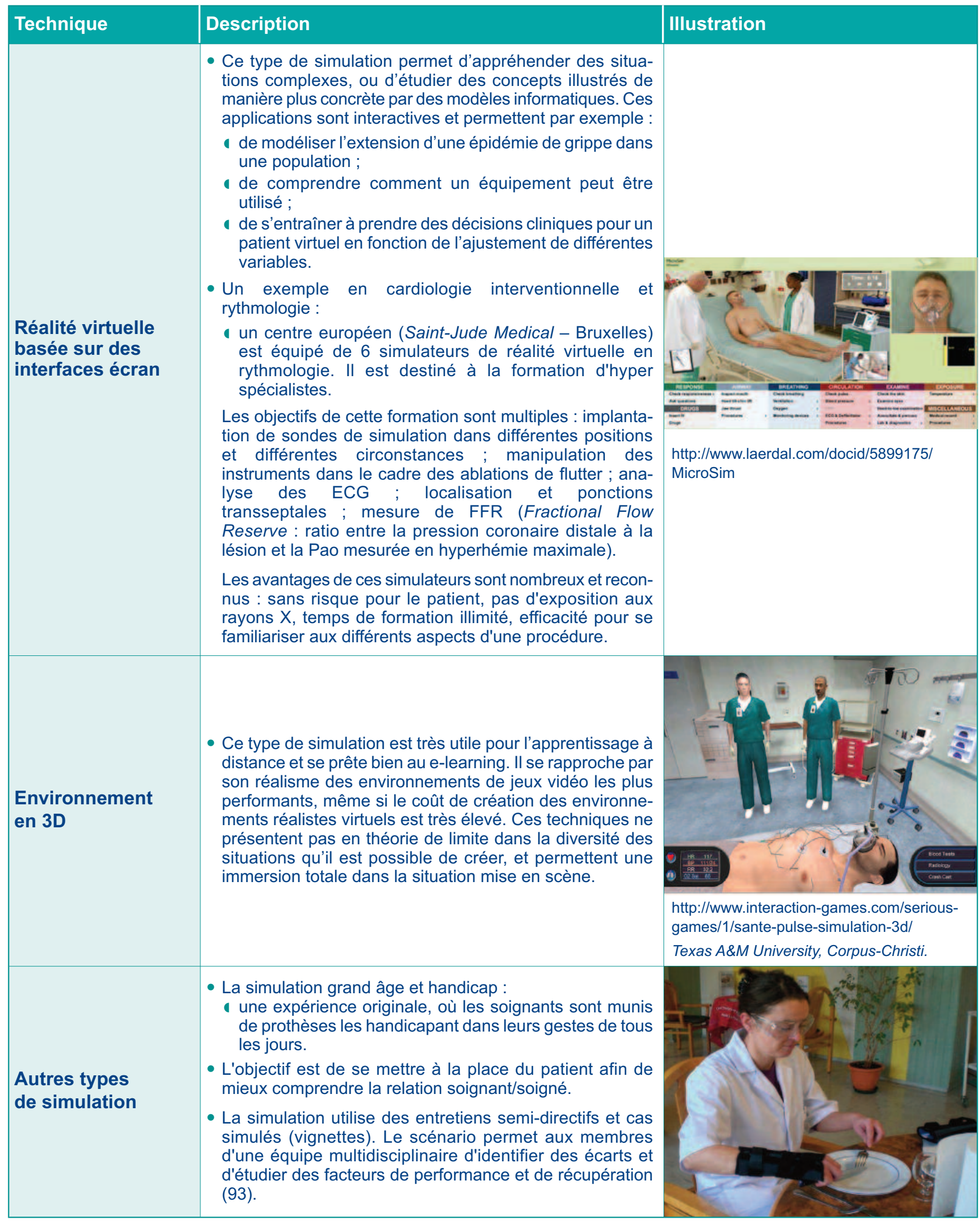




\subsection{Déroulement d'une séance de simulation (cf. figure 5)}

Ce sont les situations professionnelles, sur la base souvent de référentiels de bonnes pratiques ou d'incidents vécus, qui serviront de support à la construction des scénarios de formation. Comme le précisent Philippe Fauquet-Alekhine et al. (30), la pratique est considérée « comme objet d'analyse, l'objectif est de comprendre le mode d'action professionnel pour le mettre en débat et éventuellement le faire progresser » (94).

Plusieurs éléments doivent être pris en considération en matière de formation continue par des méthodes de simulation.

\subsubsection{En premier lieu, le simulateur}

C'est l'outil sur lequel le professionnel va s'exercer. Plus ou moins sophistiqué, plus ou moins onéreux, il sera déterminant pour le type de formation proposée. La qualité écologique du simulateur revêt une grande importance comme le signalent Pascale Béguin et Annie Weill-Fassina en 1997 (4) ; paradoxalement, on peut parfois noter que l'hyper réalisme du simulateur n'est pas forcément un gage d'apprentissage. Ce qui est déterminant, c'est l'immersion mentale du stagiaire (au-delà même du degré de réalisme de la situation), le stagiaire devant « entrer » dans la situation.

\subsubsection{Situation simulée}

Elle est supportée par un scénario, le plus souvent rédigé (annexe 3 " exemple de scénario rédigé »). Cette situation doit viser un objectif pédagogique (gestion du risque, application d'une procédure, EPP). Elle ne doit pas être inutilement compliquée pour le stagiaire. Quel que soit l'objectif visé pour un programme de formation, les scénarios devront être de difficulté croissante.

\subsubsection{Progression de la simulation}

La situation est dynamique, elle va évoluer en fonction des actions que vont réaliser à la fois les stagiaires et, en retour, les formateurs. Elle se décompose en plusieurs étapes :

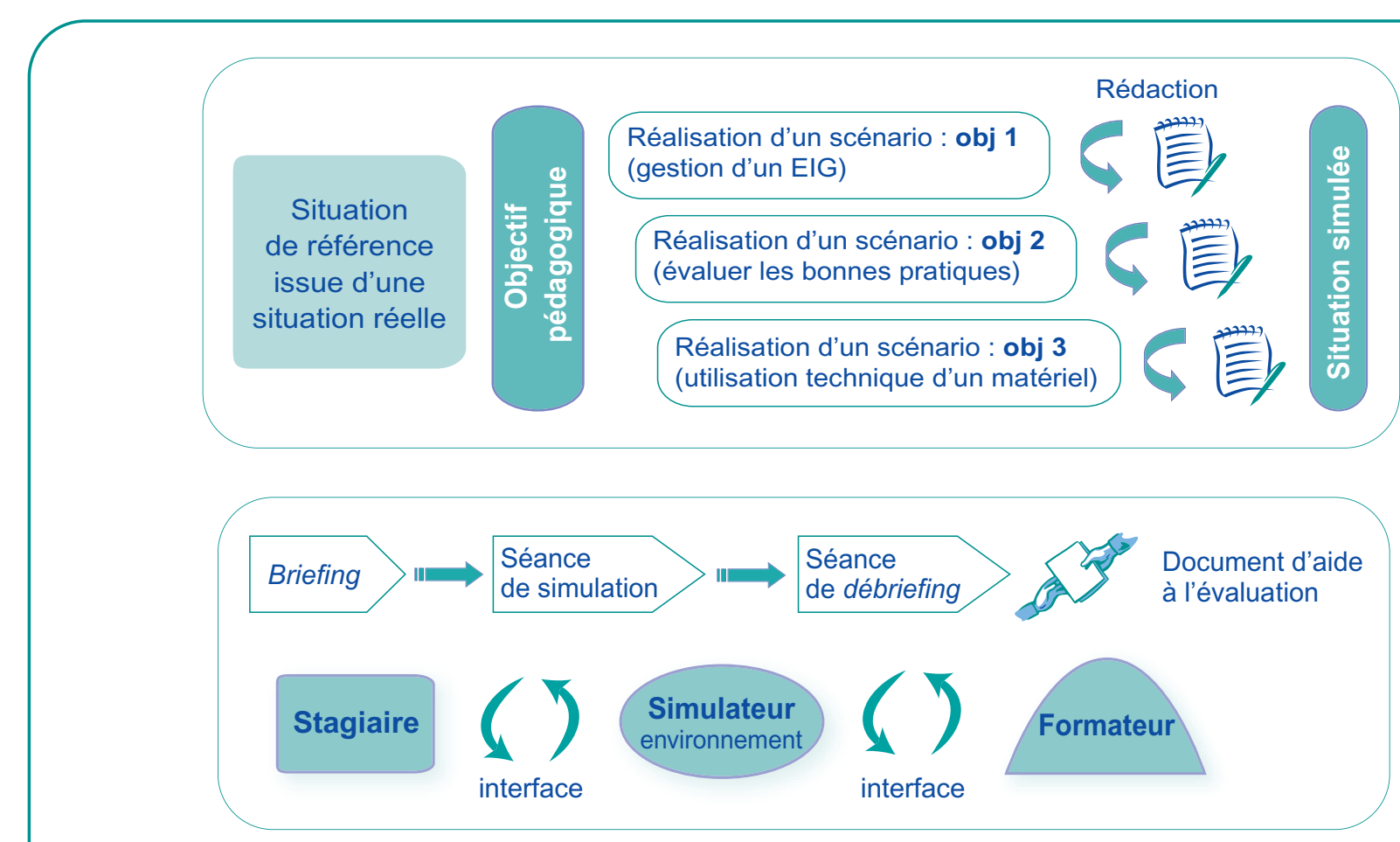

Figure 5. Diagramme d'une séance de simulation (94) Schéma inspiré de Christine Vidal-Gomel 2009 


\section{Briefing}

Comme le signale Anne-Sophie Nyssen (2005/2009) (95) dans le cadre de la simulation en anesthésie réanimation, c'est un moment important pour le bon déroulement de la séance.

En effet :

- c'est un temps indispensable de familiarisation des stagiaires avec le matériel (possibilités et limites du mannequin), de répétition d'un geste technique sur le mannequin (intubation), de présentation du dossier du patient et de contextualisation, par exemple ce qui a précédé la prise en charge simulée (passage aux urgences ou sortie de bloc opératoire, etc.). II permet aussi de présenter et de commenter l'environnement de la situation (locaux, présence de tiers ou de la famille, etc.) ;

- certains éléments seront reprécisés comme par exemple les attentes des stagiaires, de manière à éventuellement réduire le décalage entre attente du stagiaire et objectif de la formation. D'autres éléments psychologiquement importants seront aussi passés en revue, en particulier l'absence de jugement porté (ce n'est pas un examen ni une séance d'humiliation), de pièges intentionnels de la part des formateurs, et bien entendu l'absence de risque pour le « patient »!

\section{- Passage sur le simulateur}

$\rightarrow$ Le rôle du formateur et sa compétence sont essentiels à la fois pour la construction du scénario et pour le pilotage de son évolution en fonction des réactions des stagiaires. II est donc important que celui-ci dispose d'une connaissance du métier, mais également de l'approche pédagogique par la simulation. Pour piloter, le formateur procède par ajustements permanents du scénario, afin de maintenir le stagiaire en situation de résolution de problème. À l'occasion, il peut intervenir pour aider le stagiaire ou l'orienter en particulier quand celui-ci se trouve bloqué dans la situation ou pour éviter par exemple de faire « mourir » le mannequin.

$\rightarrow$ Le formateur doit cependant faire face à une double contrainte, celle de faire évoluer le scénario et celle d'évaluer les stagiaires. Cette contrainte peut être levée grâce à un enregistrement vidéo. Lors du debriefing, il n'est pas question de revisionner entièrement la séance, donc l'équipement vidéo doit permettre de sélectionner rapidement les phases importantes de la situation. L'enregistrement ne doit jamais être utilisé pour porter atteinte à la dignité du stagiaire.

\section{- Debriefing}

En premier lieu, il est important de respecter une pause entre la séance de simulation (qui peut être très consommatrice de ressources physiques et psychologiques pour le stagiaire) et le debriefing. Cette interruption permettra aux stagiaires d'observer un temps de repos, de prendre du recul et d'échanger entre eux à chaud sur les éléments qui seront mieux verbalisés ensuite en séance.

Le debriefing est le temps le plus important de la séance de simulation. La durée du debriefing doit au moins être égale à celle de la situation de simulation, soit en moyenne entre 30 et 90' (94), mais elle peut se prolonger bien au-delà. Le debriefing comporte habituellement trois phases : la phase descriptive, la phase d'analyse et enfin la phase dite « d'application ou de synthèse ».

$\rightarrow$ Phase descriptive : dans un premier temps, le formateur rappellera les informations permettant de travailler et de s'exprimer en confiance, afin d'éliminer de nouveau l'idée de jugement et de piège. Les participants décrivent leurs impressions, ce qu'ils ont ressenti. Le formateur pose des questions simples (que s'est-il passé ? Pouvez-vous décrire le scénario ?). Les stagiaires verbaliseront les faits, les causes des actions entreprises, le comment (la pratique professionnelle) de ces actions, et surtout ce qui a été pensé mais non verbalisé, autrement dit les motivations et les intentions (quand j'ai fait cela je pensais que... ; je voulais parvenir à... ; j'ai cru que X voulait ça, etc.). Seront donc évoquées les activités suspendues, contrecarrées ou gênées un peu à la manière d'un entretien d'autoconfrontation (96). L'ensemble de ces échanges croisés doivent aboutir à un nouveau savoir. 
$\rightarrow$ La phase d'analyse est souvent la plus longue. Elle a pour but d'étudier les situations pendant lesquelles les participants à la séance ont montré des conduites inappropriées. Elle doit permettre d'identifier les forces et les faiblesses de chaque participant. La décision est basée sur l'obtention des faits, et peut être confortée par la retransmission vidéo. Les critères CRM guident souvent la discussion. II est souhaitable que l'ensemble des personnes puisse s'exprimer dans un langage non agressif en dehors de tout jugement partisan.

$\rightarrow$ La phase d'application ou de synthèse : elle permet aux participants de faire une synthèse et de rapporter ce qu'ils ont appris de la séance. II est habituel de demander ce qu'ils feraient maintenant si la même situation survenait.

\section{Deux autres éléments doivent être évoqués}

$\rightarrow$ La gestion du stress (94) : un des éléments importants des méthodes de simulation est de permettre aux stagiaires de mieux se connaître et donc de développer des méta-connaissances sur soi. Savoir parler de son stress et identifier les modalités pour en abaisser le niveau permettra ainsi de mieux faire face ultérieurement en situation réelle. Il est donc important de l'aborder lors du debriefing.

$\rightarrow$ La gestion de l'échec : dans une optique pédagogique, une séance de simulation ne doit pas se solder par un échec, en particulier dans le monde de la santé où le décès du patient peut représenter l'échec ultime. La simulation ne doit donc pas être une méthode de démonstration de "l'incompétence ».

Le bon déroulement d'un debriefing dépend bien entendu de facteurs importants tels que : le niveau de compétence des participants et des formateurs, les objectifs d'apprentissage, la crédibilité des scénarios, la durée de la séance, etc.

\section{Évaluation de la séance}

La simulation, se situant dans le contexte du développement professionnel continu, doit comporter des éléments d'évaluation. Ceux-ci porteront sur les acquisitions en simulation et comporteront un système d'évaluation du progrès réalisé.

Un document de fin de séance sera proposé au stagiaire et conjointement validé (document d'aide à la progression) présentant les axes de progrès, l'orientation des formations à venir, l'évaluation de ce qui a été acquis, éventuellement des mesures d'impact sur la pratique suite à la formation. Les programmes de DPC réalisés pourront intégrer ces différents éléments.

\section{Critères qualifiant une méthode pédagogique par la simulation}

$\rightarrow$ Un objectif pédagogique.

$\rightarrow$ Un scénario bâti et rédigé visant cet objectif.

$\rightarrow$ Un briefing de présentation du contexte et de l'équipement.

$\rightarrow$ Un environnement réaliste (validation de la capacité de l'environnement à atteindre l'objectif pédagogique : équipement, réalisme, matériel, mannequin).

$\rightarrow$ La séance de simulation filmée.

$\rightarrow$ Un debriefing structuré et encadré.

$\rightarrow$ Un document de fin de séance ou fiche d'aide à la progression. 


\subsection{Formation et simulation : revue de la littérature}

La synthèse bibliographique s'est appuyée sur les résultats obtenus à partir de la stratégie de recherche documentaire suivante :

La base de données Medline (via Pubmed) a été interrogée sur la période 2000-2011 en anglais et en français à partir de l'équation de recherche :

$\rightarrow$ "Manikins"[Mesh] or "Patient Simulation"[Mesh] OR "Computer Simulation"[Mesh] OR simulation[title] OR simulator[title] OR simulated[title] AND "Education, Continuing"[Mesh] OR "Inservice Training"[Mesh] OR Continu* professional development[title/abstract] OR continu* professional education[title/abstract] OR continu* education[title/abstract] OR train*[title/abstract] OR Continu* professional development[title/abstract] OR continu* professional education[title/abstract] OR educat*[title/abstract].

$\rightarrow$ AND ("Meta-Analysis as Topic"[Mesh] OR "Meta-Analysis "[Publication Type] OR "Review Literature as Topic"[Mesh] OR "Comparative Study" [Publication Type] AND "Randomized Controlled Trial" [Publication Type] OR "Random Allocation"[Mesh] OR "Randomized Controlled Trials as Topic"[Mesh] OR "Randomized Controlled Trial "[Publication Type] OR Guidelines as Topic[MeSH] OR Practice Guidelines as Topic[MeSH] OR Health Planning Guidelines[MeSH] OR Guideline[Publication Type] OR Comparison[title/abstract]

$\rightarrow$ OR comparative[title/abstract] OR Versus[title/abstract] OR Meta-Analysis[title/abstract] OR Review Literature[title/abstract] Or Quantitative Review[title/abstract] OR Random*[title/abstract] Or guidelines[title/abstract] OR before/after stud*[title/abstract] OR before after study[title/abstract] OR before/after tria/*[title/abstract] OR interrupted time series[title/abstract] OR controlled study[title/abstract] OR evidence [title/abstract]).

$\rightarrow$ Au total, 488 références ont été obtenues.

La littérature grise a été recherchée selon trois catégories à un niveau international

$\rightarrow$ Les études scientifiques et les revues de littérature publiées et répertoriées sur le sujet.

$\rightarrow$ Les guides et les rapports publiés d'institutions, d'associations ou de sociétés savantes.

$\rightarrow$ Les documents uniquement mis en ligne sur le web, les autres documents non publiés.

En complément les sommaires des revues pertinentes ont été consultés

$\rightarrow$ Annals of Internal Medicine, Archives of Internal Medicine, British Medical Journal, Canadian Medical Association Journal, JAMA, Lancet, New England Journal of Medicine, Presse Médicale, International journal for quality in health care, Joint commission journal on quality and patient safety, BMJ Quality and Safety, Simulation in Healthcare.

II parait important de rapporter ici les méthodes et résultats d'une revue systématique conduite par l'Agency for Healthcare Research and Quality (AHRQ - États-Unis) en 2007 (97). L'objectif de ce travail était de réaliser une synthèse des preuves de l'efficacité de la Formation médicale continue (FMC), en termes d'acquisition de connaissances, de compétences techniques, de comportements et d'évaluation de la pratique clinique.

Des questions spécifiques avaient été formulées auprès d'experts qui devaient y répondre en tenant compte des données de la littérature. Plusieurs bases de données avaient été étudiées (MEDLINE, EMBASE, Cochrane Database, Cochrane Central Register of Controlled Trials and the Educational Resource Information Centre ERIC).

Parmi 68000 références identifiées dans la littérature, seulement 136 articles et 9 revues avaient été retenus. Le phénomène était expliqué par le fait que beaucoup d'articles étaient de pauvre qualité scientifique, et qu'aucune conclusion ne pouvait en être tirée.

Cependant, parmi les travaux sélectionnés, les résultats suivants pouvaient être retenus : 


\subsubsection{Intérêt de la simulation pour l'enseignement des compétences techniques}

Trois revues ont évalué l'intérêt de la réalité virtuelle pour l'enseignement des gestes chirurgicaux. Une méta-analyse (98) démontre que l'entraînement des gestes en réalité virtuelle diminue le temps nécessaire à la formation. Une autre revue (99) rapporte des résultats discordants : 2 études montrent l'amélioration de la formation chirurgicale après entraînement sur simulateur, tandis que deux autres ne décrivent aucune différence.

Une troisième revue (40) découvre un avantage de la simulation par rapport à l'enseignement standard dans ce type de formation. L'enseignement par vidéo n'amène pas de modification notable.

Une revue (100) évalue l'intérêt de la simulation par la formation en endoscopie digestive ; celle-ci a un intérêt pour le confort du patient, mais les preuves sont insuffisantes pour affirmer une amélioration de la formation.

\subsubsection{Intérêt de la simulation pour l'acquisition des connaissances}

Une revue de la littérature (5), rassemblant 33 études, démontre l'efficacité de la simulation dans ce domaine.

Une autre revue (101), concernant l'enseignement de la traumatologie, ne met pas en évidence d'avantages par rapport à l'enseignement traditionnel.

\subsubsection{Intérêt de la simulation pour la formation à la communication}

Une revue de la littérature $(101,102)$ concernant la cancérologie met en évidence de nets avantages de la formation par simulation chez des étudiants pour l'annonce de la maladie ou d'une mauvaise nouvelle.

Une seconde revue (103) découvre également la supériorité de cette formation lors de consultations de tabacologie (conseils pour cesser de fumer chez des patients standardisés).

\subsubsection{Bonnes pratiques de la formation par simulation}

Plusieurs revues de la littérature ont été réalisées concernant ce sujet. Parmi elles, deux sont particulièrement dignes d'intérêt.

La première (2) a étudié cinq bases de données par l'intermédiaire de quatre-vingt-onze mots-clés sur une période de trente-cinq ans (1969-2003). Durant cette période, les auteurs ont retenu cent neuf études selon les quatre critères suivants : élimination des articles réalisés avec une méthodologie empirique ; utilisation d'un simulateur pour une évaluation objective des apprenants avec des résultats quantitatifs ; travaux de recherche comparatifs, expérimentaux ou quasi expérimentaux ; travaux de recherche impliquant la simulation comme unique méthode de formation. Les données des cent neuf articles ont été analysées par neuf lecteurs indépendants, selon un protocole standardisé.

Les résultats rapportés tiennent compte essentiellement des implications de ces travaux pour la pratique et selon les principes de l'Evidence Based Medicine. Ils peuvent être résumés ainsi :

- le debriefing est une étape essentielle de la simulation (47\% des articles retenus);

- de même que la pratique répétée (39\% des articles) ;

- l'intégration de la simulation dans le curriculum global de formation (initiale ou continue) est un autre point majeur ( $25 \%$ des articles cités) ;

- la formation doit être réalisée avec des niveaux de difficulté croissants (14\%);

- en utilisant plusieurs stratégies d'apprentissage (10\%);

- et la possibilité de reproduire des situations cliniques variées (10\%); 
- dans un environnement contrôlé (où les apprenants peuvent faire, détecter ou corriger des erreurs sans risque) (9\%) ;

- les expériences pédagogiques avec la simulation doivent être reproductibles, standardisées et impliquant activement les participants ( $9 \%$ des articles) ;

- les objectifs pédagogiques doivent être précis et explicites, permettant des comparaisons et des résultats mesurables $(6 \%)$;

- enfin, s'assurer que le simulateur est un outil validé d'apprentissage (3\%).

La seconde revue de la littérature a été réalisée par les mêmes auteurs sur la période 2003-2009 et a été publiée en 2010 (104). Les auteurs ont cette fois choisi les articles témoignant d'une recherche de qualité et ceux permettant de préciser les critères ci-dessus exposés.

Ainsi, soixante-huit articles ont été sélectionnés permettant d'identifier et de discuter douze points caractéristiques et de bonnes pratiques de l'enseignement par simulation. Cinq de ces points avaient été retenus lors de la revue précédente; sept autres sont développés à partir des données récentes de la littérature et de la pratique. Tous ces critères représentent des éléments essentiels à considérer pour le développement professionnel continu, ils méritent d'être développés.

\section{Les 12 points caractérisant les bonnes pratiques de la formation par simulation}
1. Debriefing
7. Pédagogie de maîtrise (ou par objectifs)
2. Pratique « délibérée "
8. Transfert des bonnes pratiques
3. Intégration dans le curriculum
9. Travail en équipe
4. Évaluation des formations
10. Examens sanctionnants
5. Fidélité de la simulation
11. Formation des formateurs
6. Acquisition et entretien des connaissances
12. Contextes éducationnels et professionnels des apprenants

\section{Debriefing}

Pour de nombreux auteurs, le debriefing est la partie la plus importante de l'apprentissage par simulation. Il doit être structuré. En 2008, Salas et al. (105) ont décrit les douze meilleures pratiques du debriefing fondées sur des preuves :

\section{Les 12 bonnes pratiques de debriefing}

1. Les debriefings doivent avoir un intérêt diagnostique (forces et faiblesses des participants).

2. Ils doivent être réalisés dans un environnement facilitant l'apprentissage.

3. Les formateurs et participants doivent privilégier les discussions sur le travail d'équipe.

4. Les leaders doivent être formés à l'art et la science du debriefing.

5. Les membres participants doivent se sentir à l'aise durant les debriefings.

6. Le debriefing doit être focalisé sur quelques points critiques.

7. Les comportements et interactions d'équipes performantes doivent faire l'objet de descriptions ciblées.

8. Des indicateurs objectifs de performance doivent être utilisés.

9. Les résultats du debriefing doivent être fournis secondairement.

10. Le debriefing doit être réalisé à la fois au plan individuel et au plan de l'équipe au moment le plus approprié. 11. Le debriefing doit avoir lieu dès que possible après la séance.

12. Les conclusions et les buts du debriefing doivent être enregistrés pour faciliter des debriefings ultérieurs. 
Fanning et Gaba (106) ont également étudié le rôle du debriefing au cours de la formation par la simulation. Ils insistent sur le fait que les outils du debriefing sont de divers ordres : un formateur facilitateur, le matériel lui-même (mannequin) et les enregistrements vidéo. Chacun de ces éléments a des avantages et des inconvénients, mais leur combinaison présente un intérêt éducationnel certain.

Plusieurs articles ont montré l'intérêt du debriefing $(107,108)$ au cours de situations cliniques critiques. Malgré ces évidences, plusieurs questions demeurent : quelles sont les caractéristiques du debriefing idéal ? Existe-t-il des techniques plus performantes et plus économes en ressources ? Des guidelines doivent être développées pour qu'en particulier les compétences des instructeurs puissent être évaluées.

\section{Pratique délibérée (109)}

La pratique délibérée est un mode d'entraînement, très souvent utilisé chez les sportifs de haut niveau et qui doit présenter au moins neuf caractéristiques quand elle est utilisée pour la formation médicale.

\section{Les 9 caractéristiques de la pratique délibérée en santé}

1. Les participants doivent être motivés et suffisamment concentrés.

2. Ils doivent s'engager pour des objectifs bien définis.

3. À chaque objectif correspond un niveau approprié de difficulté.

4. La pratique doit être ciblée, répétée.

5. Des mesures précises, rigoureuses sont conduites.

6. Un retour d'information (debriefing) bien encadré est réalisé.

7. Ce retour amène les participants à corriger les stratégies, les erreurs et leur niveau de compréhension.

8. L'objectif est d'atteindre un standard.

9. L'atteinte du standard précède l'évolution vers une autre tâche ou objectif.

L'efficacité de cette méthode éducationnelle a été montrée pour la réanimation cardio-pulmonaire $(83,110)$ ou certains gestes techniques (ponction pleurale (24), et mise en place de cathéters centraux) $(111,112)$.

\section{Intégration dans le curriculum}

Le troisième principe de la formation médicale par la simulation est l'intégration de cette méthode dans le curriculum de la formation, au même titre que les autres techniques comme la bibliographie, le travail de laboratoire, etc. Ceci signifie que l'éducation par la simulation doit être organisée, planifiée et reconnue. La simulation est complémentaire des autres méthodes de formation, mais ne peut remplacer l'expérience clinique au lit du malade. Des travaux de recherche complémentaires sont nécessaires pour préciser la place exacte de la simulation dans le cursus de formation médicale.

\section{Évaluation (mesure des résultats)}

Trois types d'évaluation de la formation par simulation peuvent aujourd'hui être envisagés : la première est l'observation de la performance des participants, mais l'interprétation de cette évaluation est souvent sujette à caution car présentant plusieurs biais. Une seconde possibilité est l'évaluation écrite des participants soit par l'intermédiaire de QCM, soit par celui d'un rapport écrit. Cette évaluation est souvent de meilleure qualité que la simple observation des pratiques. Enfin, une troisième possibilité est représentée par une mesure objective des performances comme par exemple l'utilisation de capteurs au niveau de certaines régions anatomiques d'un mannequin lors d'un examen clinique simulé. Ces méthodes très prometteuses demeurent cependant en cours d'évaluation (113).

Ces évaluations demeurent un enjeu essentiel pour préciser l'efficacité de cette formation. 


\section{Fidélité de la simulation}

Les matériels actuels utilisés demeurent encore assez éloignés de la réalité clinique, bien que certains mannequins puissent simuler des réactions " physiologiques » après gestes techniques ou administration de médicaments par exemple. Certains simulateurs utilisant la réalité virtuelle (par exemple simulateur de cœlioscopie) sont également assez proches de la réalité clinique. Plus récemment, les techniques de simulation « hybride », associant matériel synthétique chez des patients standardisés (sutures chirurgicales, hémorragie simulée sur un vrai membre), présentent des caractéristiques cliniques étonnamment réalistes, et sont particulièrement efficaces pour la formation $(114,115)$.

\section{Acquisition et maintien des connaissances}

Plusieurs travaux de qualité ont montré l'intérêt de la simulation par l'apprentissage des gestes techniques en particulier $(82,112,116)$.

Le maintien de ces connaissances est variable selon les publications. II dépend du type de connaissances acquises, du degré d'apprentissage, du temps écoulé entre la phase d'apprentissage et l'évaluation secondaire.

Les données de la littérature sont aujourd'hui insuffisantes pour pouvoir conclure.

\section{- Pédagogie par objectifs}

Le but de cette pédagogie est de s'assurer que tous les apprenants réalisent l'ensemble des objectifs éducationnels avec peu de variations entre eux.

Cette méthode présente schématiquement cinq caractéristiques (117) :

1. Évaluation initiale.

2. Objectifs clairement définis, sous forme de modules de difficulté croissante.

3. Utilisation des moyens pour atteindre ces objectifs (lecture, entraînement, etc.).

4. Évaluation minimale pour chaque module (test score).

5. Passage au module suivant seulement si les objectifs prévus sont atteints.

\section{- Transfert à la pratique clinique}

Les travaux permettant de confirmer l'intérêt direct de la simulation pour la pratique clinique sont de réalisation difficile.

Plusieurs études sont cependant convaincantes: Wayne et al. (110) ont montré que des internes en médecine formés par la simulation sont plus performants pour la prise en charge d'arrêts cardiaques intrahospitaliers. Une autre étude démontre également que des internes, formés par simulation à la mise en place de cathéters veineux centraux, ont moins de complications (ponctions artérielles) que ceux qui n'ont pas été formés par cette méthode (84). De même, les infections liées aux cathéters seraient moins fréquentes quand ces derniers ont été mis en place par des personnels formés par simulation (85). En chirurgie, il a été montré que la formation par simulation (réalité virtuelle) améliore notablement la performance des opérateurs (118). Il en est de même en obstétrique lors d'accouchements compliqués de dystocie des épaules. Les praticiens formés par simulation obtiennent de meilleurs résultats (86). Des travaux complémentaires de méthodologie très rigoureuse sont à entreprendre pour confirmer ces résultats d'importance.

\section{- Formation d'équipes}

La notion d'équipe est essentielle en matière de prise en charge des patients. Les dysfonctionnements à l'intérieur des équipes soignantes sont responsables de nombreux effets indésirables. Cette formation serait notablement améliorée par la simulation (119). Un des objectifs majeurs est ici d'évaluer la performance des équipes par la simulation. 


\section{Évaluations sanctionnantes}

L'utilisation de la simulation pour l'évaluation sanctionnante est de plus en plus fréquente. C'est le cas dans certaines disciplines (anesthésiologie) et dans certains pays (Israël, Australie, Nouvelle-Zélande) (120-122). Aux États-Unis, la simulation est utilisée pour l'évaluation de praticiens étrangers qui souhaitent exercer dans ce pays. Cette évaluation porte sur les compétences techniques et non techniques (comportement, communication, etc.).

\section{Formation des formateurs}

Cette formation est aujourd'hui absolument indispensable. Cette compétence n'est pas innée. L'expérience clinique seule n'est pas suffisante pour se déclarer formateur. Plusieurs travaux ont été publiés sur le sujet $(104,123,124)$, mais la question justifie une réflexion approfondie par les responsables institutionnels afin d'apporter des formations de qualité.

\section{- Contexte éducationnel et professionnel des apprenants}

Cette notion est essentielle pour la réussite de la formation. Celle-ci doit être adaptée aux souhaits et objectifs professionnels des participants. Elle doit être aussi facilitée par l'institution.

\subsection{Simulation : un outil de développement professionnel continu et de gestion des risques}

II s'agit là de l'objet principal de cette étude, puisque celle-ci s'intéresse à la formation par la simulation dans le cadre du DPC (seront considérés comme outils de DPC la formation professionnelle traditionnelle ainsi que les outils d'EPP, comme précisé dans la loi HPST 2009). Dans ce cas, le professionnel possède les compétences utiles au métier, et pour la grande majorité, ces éléments sont acquis (médecins seniors).

La question de la formation continue en santé amène à plusieurs constats :

$\rightarrow$ Premier constat : une structuration réglementaire progressive mais importante du dispositif de Formation médicale continue (FMC) et d'Évaluation des pratiques professionnelles (EPP) depuis une dizaine d'années :

"Les médecins quel que soit leur mode d'exercice ont le devoir de se former et d'évaluer leurs pratiques ». Cette obligation s'est traduite par une succession de textes. Les décrets de 1995 intégrés au Code de la santé publique ${ }^{7}$, puis les ordonnances de $1996^{8}$, renvoyaient à une obligation de FMC et d'EPP. La loi du 13 août 2004, relative à l'Assurance maladie dans son article 14, faisait obligation aux médecins d'EPP individuelle, et l'article 16 présentait l'accréditation volontaire des médecins (basée sur la déclaration des situations à risques ou événements porteurs de risques).

D'autres textes se sont succédés jusqu'aux arrêtés du 13 juillet 2006, homologuant les règles de validation de la FMC. Ce dispositif un peu complexe, basé sur une comptabilité fastidieuse de points de FMC et d'EPP, a ensuite été modifié par la loi HPST de 2009, transformant l'obligation de FMC pour les seuls professionnels médicaux en obligation de développement professionnel continu (DPC) opposable à tous les professionnels de santé.

Par ailleurs, la certification des établissements de santé dans ses versions successives V2005 et V2010 a rendu obligatoire l'EPP collective au sein de ceux-ci, moyennant l'utilisation d'outils validés par la HAS.

$\rightarrow$ Deuxième constat : dans leur rapport pour l'inspection générale des affaires sociales de novembre 2008, Pierre-Louis Bras et Gilles Duhamel (125) montraient que « dès lors que la formation emprunte des formes pédagogiques didactiques traditionnelles, celle-ci a peu d'effet direct sur les pratiques. Les modes de formation plus interactifs et plus ancrés dans la pratique effective des médecins auraient un impact plus prononcé ». L'évaluation des pratiques et la simulation font partie de ces méthodes.

7. Article 11 du Code de déontologie (décret $n^{\circ}$ 95-1000 de septembre 1995) « Tout médecin doit entretenir et perfectionner ses connaissances ; il doit prendre toute disposition nécessaire pour participer à des actions de formation continue, tout médecin participe à l'évaluation des pratiques professionnelles ".

8. Ordonnance $n^{\circ}$ 96-345 du 24 avril1996 faisant de la FMC une obligation légale. 
$\rightarrow$ Troisième constat : les méthodes de simulation permettent l'acquisition de savoir, de savoir-faire et de savoir-être qui impactent fortement - même si cela n'a pas réellement été quantifié - la performance et la sécurité de la prise en charge des patients. Ces méthodes sont, par exemple, largement utilisées et obligatoires dans l'aviation civile pour l'évaluation régulière des pilotes. Même s'il n'a pas été démontré que ces évaluations diminuaient le nombre d'accidents.

En l'absence de données sur l'impact de ce type de formation concernant la qualité de la prise en charge, il est possible néanmoins d'utiliser un modèle d'évaluation des formations proposé par Donald Kirkpatrick $(126,127)$. Ce modèle propose 4 niveaux.

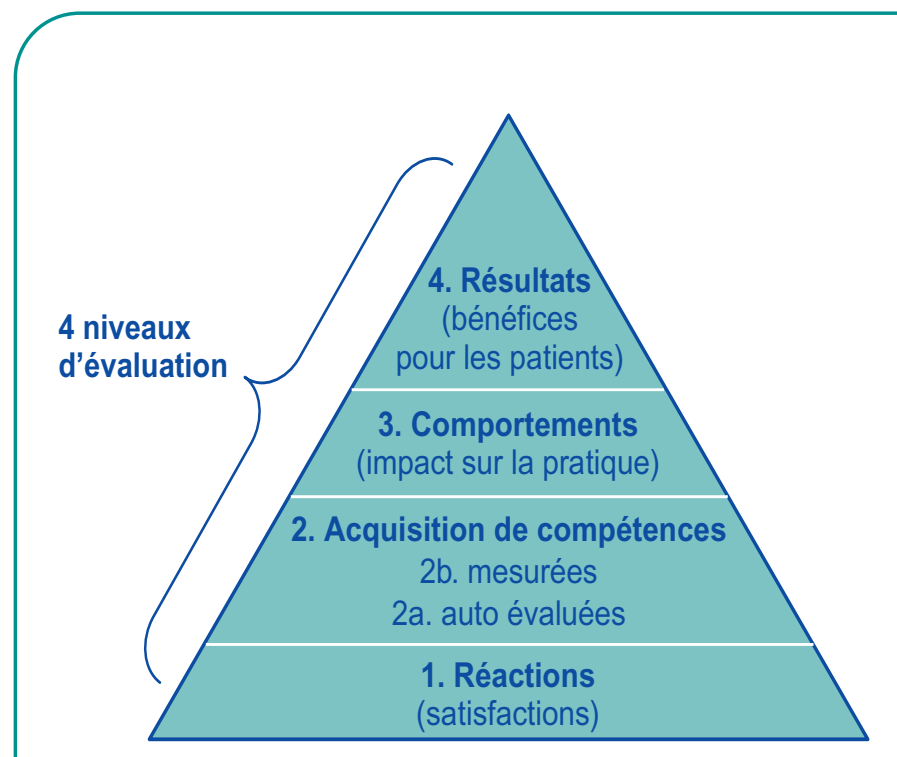

\begin{abstract}
$\rightarrow$ Niveau 1
Réactions : Comment ont réagi les formés à l'issue de la formation ? Ont-ils apprécié celle-ci ? En sontils satisfaits?
\end{abstract}

\title{
$\rightarrow$ Niveau 2
}

Apprentissage : Qu'ont appris les formés à l'issue de la formation? Quelles connaissances, habiletés et/ou attitudes (savoir, savoir-faire, savoir-être) ont été acquises ? Les objectifs pédagogiques ont-ils été atteints ? II s'agit ici de l'évaluation pédagogique.

\section{$\rightarrow$ Niveau 3}

Transfert : Est-ce que les formés utilisent ce qu'ils ont appris en formation à leur poste de travail ? Quels comportements professionnels nouveaux ont été adoptés?

\section{$\rightarrow$ Niveau 4}

Résultats organisationnels : Quel est l'impact de la formation sur la prise en charge des patients ?

Figure 6. Modèle modifié de Kirkpatrick (1967)

En ce qui concerne la formation par la simulation, il est aujourd'hui prouvé qu'elle répond positivement aux niveaux 1, 2 et 3 ; des éléments de preuve sont encore nécessaires pour affirmer que la simulation améliore indiscutablement la qualité des soins fournis aux patients.

On peut donc penser qu'une combinaison des méthodes d'EPP et de simulation pourrait représenter un bon compromis, à la fois en termes de conséquences pédagogiques, mais aussi d'intérêt des professionnels dans le cadre des démarches de DPC.

\subsubsection{Définition du DPC}

Il est important dans un premier temps de rappeler quelques définitions à la fois celle de l'EPP et celle du DPC.

Dans un premier temps, la HAS définit l'EPP. Celle-ci consiste en l'analyse de la pratique professionnelle en référence à des recommandations selon une méthode élaborée ou validée par la Haute Autorité de Santé, et inclut la mise en œuvre et le suivi d'actions d'amélioration des pratiques.

« L'évaluation des pratiques professionnelles a pour but l'amélioration continue de la qualité des soins et du service rendu aux patients par les professionnels de santé ».

« Elle vise à promouvoir la qualité, la sécurité, l'efficacité et l'efficience des soins et de la prévention et plus généralement la santé publique, dans le respect des règles déontologiques ». 
Cette évaluation n'est jamais sanctionnante, mais se veut totalement formative.

Dans un second temps, la loi HPST confirme l'obligation de formation continue et d'EPP, sous le terme unifié de Développement professionnel continu (DPC). Le DPC est défini dans l'article 59 de la loi HPST comme : " l'évaluation des pratiques professionnelles, le perfectionnement des connaissances, l'amélioration de la qualité et de la sécurité des soins ainsi que la prise en compte des priorités de santé publique et de la maîtrise médicalisée des dépenses de santé ».

Les décrets d'application, définissant les modalités d'application de ce dispositif, et la validation de leur DPC par les professionnels sont parus le 30 décembre 2011.

\subsubsection{Caractéristiques des projets de DPC}

De tels projets s'appuieront donc sur la définition qu'en fait la loi, et engloberont à la fois le champ de la qualité et de la gestion des risques. Cependant, pour être qualifié d'EPP, un projet doit présenter un certain nombre de caractéristiques lui permettant de répondre à cette définition (128). II sera donc nécessaire d'y trouver :

- un enjeu d'amélioration de la qualité permettant de dégager une marge significative de progrès ;

- une analyse de pratique en lien avec l'activité du professionnel concerné. S'il s'agit d'un médecin, ce projet doit permettre d'évaluer son activité clinique spécifique ;

- la prise en compte de références validées comme par exemple des "Bonnes Pratiques Cliniques » regroupées au sein d'un référentiel cosigné par la HAS et une société savante, une conférence de consensus, une revue de la littérature, etc. ;

- un outil validé par la HAS : aujourd'hui de nombreux outils sont décrits et disponibles sur le site de la $\mathrm{HAS}^{10}$. Ils sont utilisables in extenso, mais peuvent aussi être adaptés de manière à s'intégrer aux activités de soins ;

- la mesure d'un résultat qui signe l'amélioration effective de la pratique ;

- l'évaluation des pratiques emprunte à la qualité et à la gestion des risques de nombreux outils et, par ailleurs, se déploie selon des approches de type amélioration de la qualité ou gestion des risques. Certaines sont citées dans le tableau 9 (§5.4.4).

Compte tenu de tous ces éléments, la simulation couvre l'ensemble du champ du DPC. En effet, celleci peut permettre aussi bien la formation procédurale à des gestes ou des situations techniques (formation classique d'apprentissage) que l'évaluation des pratiques professionnelles et des synergies d'équipe ou d'améliorer la sécurité des soins. Nous nous attacherons donc à présenter les outils utilisables en simulation pour ces différentes approches.

\subsubsection{Applications de la simulation dans la gestion des risques et le DPC}

Depuis plusieurs années maintenant, la sécurité sanitaire fait partie à juste titre des priorités politiques. En 2000, le rapport de l'institut américain de médecine avançait que 4 à $16 \%$ des patients subissaient des effets indésirables liés aux soins, et que les décès rapportés aux erreurs " médicales " se situaient entre 44000 et 98000 personnes par an aux États-Unis. Ces chiffres ont été ensuite confirmés par plusieurs études issues de différents pays. En France, la direction de la recherche, des études, de l'évaluation et des statistiques (DRESS) a réalisé en 2004 une grande enquête nationale sur un échantillon d'établissements représentatifs, pour y identifier la prévalence des effets indésirables graves (EIG). Les événements les plus graves et les plus fréquents étaient observés au sein du circuit des produits de santé (médicaments, dispositifs médicaux), après des actes invasifs dont les interventions chirurgicales et suite à des infections nosocomiales. En 2009, la deuxième itération de cette enquête recensait, sur 8262 séjours observés, dans 251 unités de soins au sein de 81 établissements, 374 EIG dont 177 étaient évitables. Soit en 
moyenne 1 tous les 5 jours (129) Parmi les causes les plus fréquemment identifiées, on retrouvait en particulier celles liées au facteur humain (manque de communication, absence de supervision, dérangement dans la tâche, etc.).

En aéronautique, comme dans d'autres activités à risque, l'étude des facteurs humains a fait l'objet de formations spécifiques. Ainsi, les pilotes ont pu bénéficier de séances d'entraînement sur simulateurs, leur permettant d'affronter nombre de situations à risque, sans danger pour leurs passagers. De même, la formation des personnels de santé par la simulation a fait l'objet de nombreux travaux qui démontrent l'intérêt de cette méthode pédagogique dans l'amélioration de la qualité des soins et la limitation des EIG.

\section{Fiabiliser les pratiques : simuler les accidents pour apprendre de ses erreurs}

Dans l'aéronautique comme dans le domaine de la santé, il est possible de comprendre les événements indésirables grâce à des analyses de causes rétrospectives utilisant les méthodes de retour d'expérience. Ceci se fait lors des revues dites de " mortalité-morbidité ou RMM » (revue périodique des événements iatrogènes morbides ou létaux). II existe des liens entre RMM (outil d'EPP et de gestion des risques) et simulation. Dans les deux cas il s'agit, à partir de l'histoire d'un événement indésirable, souvent grave, d'analyser les causes, d'identifier celles qui seraient évitables, de confronter la discussion à la littérature et de mettre en place des actions correctives évitant que la situation ne se reproduise. La RMM peut être utilisée en simulation (130).

Trois options de mise en œuvre peuvent être proposées :

- bâtir les scénarios à partir de la banque d'incidents et comparer comment plusieurs équipes se comportent ;

- bâtir les scénarios à partir de la banque d'incidents et comparer la solution retenue par l'équipe ayant vécu la situation réelle et celle qui l'expérimente en simulation ;

- réaliser une reconstitution d'accident avec une équipe d'acteurs et induire l'analyse de causes et la discussion à partir du cas illustré en direct.

La mise en situation simulée de la RMM comporte un avantage distinctif sur l'identification des actions d'amélioration. En effet, celles-ci porteront à la fois sur l'amélioration des connaissances techniques, des compétences, mais aussi de la connaissance de soi et des synergies d'équipes. En cela elles constituent un outil d'évaluation formative et de fiabilisation basée sur le retour d'expérience. Par le partage vécu de la situation, la simulation permettra au plus grand nombre d'améliorer la capacité à faire face si une situation identique se reproduit.

\section{- Développer chez les professionnels la capacité de détection et de récupération des erreurs, la synergie d'équipe, les attitudes sécuritaires (131)}

La simulation peut permettre de développer ces stratégies chez les personnels de santé, d'analyser et de promouvoir des modes de résilience. La simulation est citée par P. Roussel, M-C. Moll et P. Guez en 2007 comme outil de gestion des risques a priori (132). Certaines méthodes apparaissent essentielles à mettre en œuvre, qu'elles soient individuelles ou collectives pour développer la fiabilité humaine. Des pratiques de "fiabilités humaines » ont été développées dans les industries dites ultra sûres tel le nucléaire (EDF en France) ou l'aéronautique ou encore les industries du pétrole. II est admis que l'erreur étant humaine (13), il est nécessaire de développer des stratégies pour les récupérer ou les anticiper. Ces pratiques de fiabilité ou attitudes sécuritaires ${ }^{11}$ peuvent être détectées en simulation et même servir de fil conducteur à l'analyse du scénario en termes de gestion des risques. La simulation permet aussi d'entraîner les équipes à développer ces comportements sécuritaires ( "Programme d'entraînement pour la check-list »par exemple. Annexe 5). Quelques exemples de ces pratiques de fiabilité sont cités ci-dessous.

11. Publication des BPS, site MEAH et AFM. 


\section{$\rightarrow$ Attitudes individuelles}

\section{Minute d'arrêt}

Pendant l'exécution d'une tâche sensible en matière de sécurité, l'opérateur doit pouvoir refuser de se laisser interrompre, sinon il doit gérer l'interruption de la tâche en utilisant la « minute d'arrêt », et :

- repérer le point précis de son interruption ;

- reprendre l'identification complète des éléments de la tâche ;

- recommencer au point précis de l'interruption (si inconnu, reprendre entièrement la procédure).

\section{Autocontrôle}

II s'agit du contrôle par l'exécutant lui-même de l'action qu'il va réaliser. Cette vérification est un acte professionnel : l'agent suit la procédure à haute voix, et vérifie en parallèle à haute voix les différentes étapes de la tâche. Cela garantit l'adéquation entre action prévue et action réalisée, et permet de mobiliser l'attention exclusivement sur la tâche.

Cette attitude doit être utilisée en particulier si l'action comporte un enjeu fort ou si l'acte est complexe avec des conséquences potentielles graves ou irréversibles pour le patient.

\section{$\rightarrow$ Attitudes collectives : les synergies d'équipe}

\section{Briefing}

Les professionnels doivent, avant la réalisation de la tâche, dialoguer pour s'organiser, pour définir les points de surveillance, les parades possibles aux risques, les interactions entre eux, le retour d'expérience dans une situation similaire vécue antérieurement.

\section{Contrôle croisé}

II est important d'utiliser cette méthode quand la réalisation d'actions ou de séquences d'actions peut aboutir à des conséquences graves, si elles sont réalisées de façon incorrecte et pour lesquelles il n'y a pas de marche arrière possible. Cela permet de bénéficier du regard et du contrôle (bienveillant) de l'autre, avant action ou saisie d'un paramètre critique. C'est le dernier regard d'un tiers avant validation (dernière chance, ou barrière).

\section{Check-list}

Cette liste permet de vérifier que les actions essentielles prévues ont bien été effectuées. Avant l'action, il s'agit de vérifier que les prérequis indispensables à la sécurité sont bien disponibles. Avant une action irréversible, il s'agit de s'assurer collectivement une dernière fois de la bonne configuration du système.

\section{Communication sécurisée}

Il s'agit d'une action de communication (geste ou parole) qui permet de s'assurer que le message transmis a bien été compris.

Cela permet de garantir la transmission orale d'une information claire, complète et ciblée (les 3 C). Cela renforce la mémorisation de l'intervenant. La pratique des $3 \mathrm{C}$ est totalement nécessaire pour tout transfert d'informations critiques (bloc opératoire, réanimation, etc.). 


\section{No go}

II s'agit là d'autoriser l'arrêt sur simple doute de l'opérateur, de permettre à chacun de pouvoir interrompre le processus en cours et de poser la simple question : « j'ai un problème » " j'ai un doute » " je ne maîtrise plus ». Être " autorisé » à douter donne confiance aux opérateurs pour se permettre d'interrompre un processus. Cela permet d'éviter la « tunnellisation » qui pousse à poursuivre dans une mauvaise stratégie diagnostique ou thérapeutique malgré les alertes (erreur de fixation). Cela permet aussi de faire appel à des ressources extérieures (appel à l'aide).

\section{Délégation contrôlée}

Lors d'une délégation de tâche, c'est la démarche du « rendu compte ». Le contrôle est réalisé par les deux acteurs, la responsabilité reste au délégataire. Cela donne, en toute sécurité, une latitude pour certaines délégations de tâches et en particulier autorise le délestage en cas de surcharge de travail lors de situations particulièrement tendues. Ceci n'est bien sûr réalisable que lorsque la délégation est réglementairement possible ou lors de la formation initiale.

\section{Travail en binôme et répartition des tâches}

Quand plusieurs opérateurs sont impliqués dans une action, la répartition des tâches doit être réfléchie et formalisée. Cela permet de travailler en toute sécurité afin d'éviter les doublons et les oublis, d'optimiser la charge de travail de chacun et de rendre synergique le travail en équipe. Cette organisation du travail est particulièrement nécessaire lors de toute activité avec plusieurs acteurs ou entre opérateurs confirmés et novices.

\section{Debriefing de l'activité}

Comme dans la réalité, il permettra de vérifier :

- si les tâches ont été réalisées comme prévu ;

- si les procédures étaient correctes et facilement utilisables par un novice ;

- si les moyens et matériels étaient suffisants ;

- si tous les risques avaient bien été évalués ;

- si des éléments peuvent être améliorés pour la prochaine fois.

C'est à ce moment que pourront être analysés les éléments d'une bonne synergie d'équipe (14) qui repose sur quatre piliers principaux :

- un leader qui fixe objectifs et priorités, décide, partage les informations, délègue, écoute les remarques de ses assistants ;

- un objectif commun clairement partagé ;

- un mode de fonctionnement défini ;

- le soutien des assistants qui informent, surveillent, proposent, réagissent s'ils constatent une anomalie.

\section{$\rightarrow$ Gestion d'une crise médicale}

On doit considérer un aspect aussi important que la compétence technique : le comportement humain et ses limites. Inspirées des travaux effectués en aéronautique dans les années 70 , les compétences non techniques nécessaires à la gestion d'une situation de crise en vol ont été adaptées à la pratique médicale et enseignées initialement en anesthésie sur simulateur sous le terme d'Anaesthesia Crisis Resource Management (ACRM) (133). La simulation haute-fidélité est un outil tout à fait adapté pour travailler ces différents aspects (Tableau 8). 
Tableau 8. Anaesthesia Crisis Resource Management (ACRM), d'après Howard et al. (133)

\begin{tabular}{|l|l|}
\hline Prise de décision et cognition & Travail en équipe et gestion des ressources \\
\hline$\rightarrow$ Connaître l'environnement de travail & $\rightarrow$ Leadership et équipe \\
$\rightarrow$ Anticiper et planifier les tâches & $\rightarrow$ Appel à l'aide précoce \\
$\begin{array}{l}\rightarrow \text { Utiliser toutes les informations disponibles et utiliser le } \\
\text { double contrôle }\end{array}$ & $\rightarrow$ Communication efficace \\
$\rightarrow$ Prévenir une erreur de fixation & $\rightarrow$ Distribution des tâches \\
$\rightarrow$ Utiliser les aides cognitives & $\rightarrow$ Mobiliser toutes les ressources disponibles \\
\hline
\end{tabular}

L'absence de leadership et une mauvaise répartition des tâches sont souvent associées à une réanimation éloignée des recommandations officielles (134). La communication, verbale ou non verbale, entre l'équipe et le leader est souvent réduite en quantité et en qualité en situation de crise (135). Plusieurs outils existent pour garantir une communication efficace en situation de crise (136). Ces techniques de communication permettent de réduire la surcharge de tâches déléguées aux infirmières pour la préparation des médicaments de réanimation, terrain favorable à des erreurs (137).

Recommandée dans les ACRM, l'utilisation de l'aide cognitive doit être encouragée. Elle est cependant encore considérée comme une aide destinée aux seuls débutants (133). En 2006, Harrison et al. retrouvent par exemple une relation significative entre la performance du traitement d'une hyperthermie maligne et l'utilisation pendant la prise en charge d'un protocole écrit (138).

\subsubsection{Mode d'emploi de la simulation comme outil de DPC}

Il est donc intéressant de réfléchir à la manière d'employer la simulation en utilisant des méthodes validées, selon les différentes approches préconisées par la HAS pour la mise en œuvre de l'EPP. Les méthodes d'EPP, issues des démarches qualité et gestion des risques, sont des outils d'évaluation formative et peuvent contribuer à alimenter le DPC. Les outils HAS correspondant seront transposés en les adaptant aux méthodes de simulation.

Cette adaptation est réalisée sur la base d'un objectif fixé en fonction du type de situation simulée. Un parallèle entre la méthode telle que présentée par la HAS (principes clés de la méthode) et sa transposition en outil de simulation est proposé pour trois outils princeps : l'audit clinique, la revue de mortalité morbidité (déjà évoqué plus haut) et le chemin clinique (tableaux 10, 11 et 12).

Tableau 9. Répartition des outils d'EPP selon les différentes approches qualité ou risque (seuls les outils sélectionnés pour leur capacité à être transposés en simulation sont cités) (139).

\begin{tabular}{|l|l|c|}
\hline Type d'approche & Outil utilisable & $\begin{array}{l}\text { Approche Qualité (Q) ou } \\
\text { Gestion des risques (GDR) }\end{array}$ \\
\hline $\begin{array}{l}\text { Approche par comparaison à un } \\
\text { référentiel et par la performance }\end{array}$ & $\begin{array}{l}\text { - Audit clinique ou audit clinique ciblé (ACC) } \\
\text { - Revue de pertinence des soins } \\
\text { Approche par les problèmes }\end{array}$ & $\begin{array}{c}\text { - Analyse de morbidité-mortalité } \\
\text { - Arbres de causes de défaillance, ALARM } \\
\text { - Méthode de résolution de problèmes }\end{array}$ \\
\hline Approche par les processus & - Chemin clinique & GDR \\
\hline Approche par les indicateurs & - Suivi dans le temps, benchmarking & Q/GDR \\
\hline
\end{tabular}


Tableau 10. Approche par la comparaison ou la performance

\begin{tabular}{|c|c|}
\hline $\begin{array}{l}\text { Outil HAS } \\
\text { Audit clinique (139) }\end{array}$ & $\begin{array}{l}\text { Objectif pour la simulation } \\
\text { Vérifier que le praticien ou l'équipe connaît les bonnes pratiques } \\
\text { qui s'appliquent à une situation clinique }\end{array}$ \\
\hline $\begin{array}{l}\rightarrow \text { Un référentiel de pratiques } \\
\rightarrow \text { Une grille d'évaluation avec des critères déclinés } \\
\text { du référentiel } \\
\rightarrow \text { Une autoévaluation basée sur une observation } \\
\text { directe et une revue de dossiers } \\
\rightarrow \text { Une mesure des écarts entre pratique de } \\
\text { référence et pratique réalisée } \\
\rightarrow \text { La mise en œuvre d'actions de réduction de ces } \\
\text { écarts (amélioration) } \\
\rightarrow \text { Deux campagnes d'évaluation pour mesurer le } \\
\text { progrès } \\
\rightarrow \text { Un champ plus ou moins large : audit clinique ou } \\
\text { audit clinique ciblé } \\
\rightarrow \text { Suivi des actions d'amélioration }\end{array}$ & $\begin{array}{l}\rightarrow \text { Identifier les bonnes pratiques se rapportant à une situation clinique } \\
\rightarrow \text { Bâtir le scénario de manière à s'assurer que les praticiens et l'équipe } \\
\text { devront obligatoirement appliquer les recommandations pour résoudre } \\
\text { le problème } \\
\rightarrow \text { Debriefer: } \\
\text { - en rappelant les éléments de bonnes pratiques, } \\
\text { - en comparant la performance de plusieurs équipes. } \\
\rightarrow \text { Identifier les points forts et les meilleures stratégies de mise en œuvre } \\
\rightarrow \text { Promouvoir les meilleures approches } \\
\rightarrow \text { Comparer les résultats entre plusieurs équipes ou entre deux séances } \\
\text { réalisées à distance } \\
\rightarrow \text { Suivi des actions d'amélioration }\end{array}$ \\
\hline
\end{tabular}

Tableau 11. Approche par les problèmes et gestion des risques a posteriori

\begin{tabular}{|c|c|}
\hline $\begin{array}{l}\text { Outil HAS } \\
\text { Revue mortalité-morbidité (RMM) (140) }\end{array}$ & $\begin{array}{l}\text { Objectif pour la simulation } \\
\text { Vérifier la capacité d'une équipe à gérer un évènement } \\
\text { indésirable grave }\end{array}$ \\
\hline $\begin{array}{l}\rightarrow \text { Charte de fonctionnement } \\
\rightarrow \text { Revue périodique multiprofessionnelle des } \\
\text { dossiers de patients ayant présenté de manière } \\
\text { inattendue des complications ou décédés } \\
\rightarrow \text { Utilisation des référentiels } \\
\rightarrow \text { Analyse des causes et de leur évitabilité (discus- } \\
\text { sion pluridisciplinaire) } \\
\rightarrow \text { Réalisation d'actions d'amélioration et de } \\
\text { sécurisation sur les causes évitables } \\
\rightarrow \text { Formalisation et partage du retour d'expérience } \\
\rightarrow \text { Suivi des actions d'amélioration }\end{array}$ & $\begin{array}{l}\rightarrow \text { Bâtir le scénario à partir du répertoire de situations incidentelles, } \\
\text { issues des revues mortalité-morbidité } \\
\rightarrow \text { Rechercher les bonnes pratiques applicables au scénario } \\
\rightarrow \text { Observer comment l'équipe résout le problème et comparer à la } \\
\text { solution retenue par l'équipe originelle } \\
\rightarrow \text { Débriefer par comparaison entre la solution retenue par l'équipe } \\
\text { originelle et l'équipe en simulation } \\
\rightarrow \text { Discuter autour de l'analyse des causes de l'incident d'origine et de } \\
\text { leur évitabilité : } \\
\text { - comparer avec la stratégie et les nouvelles causes identifiées } \\
\text { éventuellement en simulation, } \\
\text { - proposer une nouvelle pratique ou attitude sécurisée. } \\
\rightarrow \text { Suivi des actions d'amélioration }\end{array}$ \\
\hline
\end{tabular}

Remarque : l'adhésion aux mesures d'améliorations mises en œuvre se trouve considérablement renforcée par le vécu direct (en temps qu'acteurs) ou indirect (spectateur du scénario) de l'équipe de RMM. 
Tableau 12. Approche par les processus et gestion des risques a priori

\begin{tabular}{|c|c|}
\hline $\begin{array}{l}\text { Outil HAS } \\
\text { Le chemin clinique (141) }\end{array}$ & $\begin{array}{l}\text { Objectif pour la simulation } \\
\text { Standardiser une pratique de prise en charge et s'assurer de } \\
\text { l'adhésion des professionnels }\end{array}$ \\
\hline $\begin{array}{l}\rightarrow \text { Planification, rationalisation et standardisation de } \\
\text { la prise en charge pluridisciplinaire des patients } \\
\text { présentant la même pathologie } \\
\rightarrow \text { Analyse exhaustive du « processus » de prise en } \\
\text { charge existant. Critique de l'existant par rapport } \\
\text { à un référentiel et à la sécurité de prise en charge } \\
\rightarrow \text { Définition de la prise en charge optimale } \\
\rightarrow \text { Identification des critères d'inclusion des patients } \\
\rightarrow \text { Formalisation d'un « super dossier de soins » } \\
\rightarrow \text { Définition d'objectifs quantifiables journaliers } \\
\text { d'impact thérapeutique } \\
\rightarrow \text { Suivi des actions d'amélioration }\end{array}$ & $\begin{array}{l}\rightarrow \text { Conception du scénario afin d'entraîner les équipes pour permettre la } \\
\text { standardisation et la maîtrise de certaines pratiques de prise en charge } \\
\rightarrow \text { Après entraînement sur simulateur, les nouvelles procédures pourront } \\
\text { être appliquées de manière ad hoc et homogène } \\
\rightarrow \text { Des entraînements périodiques pourront être organisés, afin de vérifier } \\
\text { l'apparition de déviances ou pour permettre l'actualisation des } \\
\text { protocoles } \\
\rightarrow \text { L'impact des séances de simulation pourra être évalué sur } \\
\text { l'amélioration des indicateurs de performances des soins séquentiels } \\
\rightarrow \text { La conformité des stratégies d'inclusion des patients pourra aussi être } \\
\text { évaluée } \\
\rightarrow \text { Suivi des actions d'amélioration }\end{array}$ \\
\hline
\end{tabular}

\subsubsection{Mise en œuvre de la simulation dans le cadre du DPC}

\section{Programmes}

L'objectif de mise en place de programmes consiste à s'assurer que chaque professionnel de santé dispose d'une stratégie visant l'amélioration continue et l'évaluation périodique de ses pratiques.

Cette stratégie peut être de nature individuelle ou s'intégrer dans une démarche plus globale d'équipe, de service, de pôle ou de discipline faisant intervenir aussi les notions de management dans le domaine de l'amélioration des pratiques. II est bien évident que la simulation ne peut constituer à elle seule un programme de DPC. C'est bien la combinaison des outils et des approches individuelles et collectives qui permettra d'atteindre l'objectif. Dans cet esprit, trois exemples sont proposés faisant intervenir soit une logique où un praticien réfléchit seul à son parcours, ancré néanmoins dans les exigences de sa pratique, soit une logique plus managériale et collective dans laquelle un praticien va s'insérer, et enfin la présentation détaillée de deux programmes de DPC.

\section{$\rightarrow$ Exemple 1 : stratégie individuelle}

\section{Organisation par le praticien de son parcours formatif individuel (durée au moins $\mathbf{2}$ ans)}

Programme d'évaluation des pratiques

$\rightarrow$ Prévisionnel de séquences de simulateurs, en lien avec les objectifs d'amélioration personnelle (vu par exemple avec son organisme de DPC), du service ou du pôle.

$\rightarrow$ Intégration des autres méthodes comme la RMM, la réalisation de staff EPP ou d'études de benchmarking interéquipes ou établissements.

$\rightarrow$ Intégration des préconisations des sociétés savantes.

Formation continue conventionnelle (congrès, DU, publication, etc.) 
Le pôle anesthésie réanimation poursuit sur deux ans deux objectifs principaux : lutter contre l'iatrogénie et améliorer la prise en charge de l'urgence vitale

II met en place :

- une RMM quatre fois par an ;

- une séquence simulateur basée sur les événements marquants de la pratique identifiés en RMM ;

- la mise en place d'un scénario de traitement de l'arrêt cardiaque validant les recommandations ;

- la mise en place d'une séquence comparative entre les équipes du pôle pour la vérification de la bonne intégration d'un référentiel de pratique ;

- la validation : commission EPP CME ou organisme agréé.

\section{$\rightarrow$ Exemple 3 : exemple de programme d'EPP/DPC par la simulation}

- Autour de la consultation d'annonce en cancérologie (cf. annexes 4 et 6).

- Autour de la check-list au bloc opératoire (cf. annexes 5, 5b et 6).

\section{De I'EPP à l'évaluation des compétences}

On peut donc imaginer que dans un délai pas si lointain, des organisations professionnelles développent et valident des programmes d'évaluation des compétences, basées sur la simulation permettant d'embrasser plusieurs champs.

$\rightarrow$ La revalidation périodique des compétences des praticiens et des professionnels, tout au long de leur carrière, à l'instar des pilotes de ligne ou comme cela a été proposé par le Mont Sinaï Hospital de NewYork pour la revalidation des compétences en anesthésie grâce à un véritable programme de réentraînement (3).

Tableau 13. Six domaines de compétences pour la revalidation des pratiques en anesthésie réanimation (3), selon le ACGME (Accreditation Concil for Graduate Medical Education) et I'ABSM (American Board of Medical Specialites)

\begin{tabular}{|c|c|c|}
\hline & Domaine de compétence & Critères d'évaluation \\
\hline 1 & Professionnalisme & $\begin{array}{l}\rightarrow \text { Ponctualité } \\
\rightarrow \text { Apparence } \\
\rightarrow \text { Motivation } \\
\rightarrow \text { Bon sens }\end{array}$ \\
\hline 2 & Objectifs de communication & $\begin{array}{l}\rightarrow \text { Communiquer de façon pertinente avec les autres } \\
\rightarrow \text { Appel à l'aide (rapidité et pertinence) }\end{array}$ \\
\hline 3 & Objectifs cliniques & $\begin{array}{l}\rightarrow \text { Prise en charge des difficultés d'abord des voies aériennes } \\
\rightarrow \text { La sédation anesthésique : théorie et pratique } \\
\rightarrow \text { L'induction anesthésique : théorie et pratique } \\
\rightarrow \text { La curarisation : théorie et pratique }\end{array}$ \\
\hline 4 & Savoir médical & $\begin{array}{l}\rightarrow \text { Différent mode d'évaluation des voies aériennes (Mallampati) } \\
\rightarrow \text { Optimisation des positions (table, patient) } \\
\rightarrow \text { Contrôle de fonctionnement des équipements } \\
\rightarrow \text { Anticipation des difficultés } \\
\rightarrow \text { Hypotension et hypoxémie }\end{array}$ \\
\hline 5 & Soins & $\begin{array}{l}\rightarrow \text { Choix et utilisation pertinente des traitements } \\
\rightarrow \text { Savoir maintenir une ventilation et une oxygénation efficaces } \\
\rightarrow \text { Maîtriser les techniques d'intubation }\end{array}$ \\
\hline 6 & $\begin{array}{l}\text { Formation et évaluation } \\
\text { des pratiques }\end{array}$ & $\begin{array}{l}\rightarrow \text { Bonne utilisation des ressources } \\
\rightarrow \text { Faciliter l'apprentissage des étudiants et des autres professionnels de soins }\end{array}$ \\
\hline
\end{tabular}


$\rightarrow$ Le bilan des « savoir-faire » des nouveaux professionnels recrutés dans un établissement.

$\rightarrow$ La remise à niveau des praticiens évoluant vers une nouvelle branche de la discipline (par exemple : anesthésie adulte vers anesthésie pédiatrique).

$\rightarrow$ Le transfert de compétences d'activités médicales vers des activités paramédicales.

$\rightarrow$ La validation de nouvelles compétences acquises tout au long du cursus, la sélection des internes comme cela se pratique dans certains centres du Royaume-Uni (142), etc.

\section{Au total}

L'obligation d'EPP a constitué une opportunité majeure afin d'impliquer les praticiens et les professionnels de santé dans une dynamique d'amélioration du service « médical » rendu et de sa sécurité.

Moyennant le respect de la définition de l'EPP comme évaluation formative permettant l'amélioration de la prise en charge du patient, la combinaison aux outils de simulation permet donc de couvrir l'ensemble du champ du développement professionnel continu.

L'utilisation des simulateurs permet une véritable implication individuelle "vérifiable ", une amélioration significative de la performance individuelle et collective des professionnels confrontés à une situation de prise en charge. Si une politique concernant la formation par la simulation était définie, cela permettrait par exemple la mise à disposition de plates-formes mutualisées de formation par la simulation, autorisant ainsi l'accès à ce mode de formation au plus grand nombre de professionnels. L'enjeu majeur est de garantir une formation optimale des professionnels pour l'acquisition et le maintien de leurs compétences, dans l'intérêt des patients.

\section{Conclusion : intérêts et limites de la simulation en santé - propostions}

La pratique de la simulation en santé est devenue une méthode pédagogique incontournable. Elle est remarquable par le fait que lors de la formation, elle s'intéresse avant tout à l'apprenant mais, en même temps, son objectif prioritaire est avant tout éthique vis-à-vis du patient (" Jamais la première fois sur le patient »).

\subsection{Intérêts}

La simulation permet aux apprenants à la fois d'acquérir des connaissances, de renforcer les acquis sans risque pour le patient, de faciliter leur réflexion en groupe et d'améliorer la confiance en soi. Elle favorise l'apprentissage dit « actif ». Elle permet de reproduire une grande variété de situations rares, ainsi elle se place en tant que solution potentielle pour réduire l'écart existant entre le haut degré de compétences cliniques requis et le faible niveau d'exposition.

Plusieurs articles retrouvent un niveau de satisfaction élevé chez les participants, avec une expérience enrichissante ainsi qu'un niveau de réalisme élevé $(143,144)$. Weller signale, chez des internes formés à la pratique de l'Anaesthesia Crisis Resource Management (ACRM), que la formation en simulation a changé leur comportement : sentiment de mieux communiquer et de mieux travailler en équipe (145).

Au-delà de la satisfaction globale des participants, des compétences techniques variées peuvent être enseignées et renforcées par la simulation. Les manœuvres de réanimation cardio-respiratoire sont grevées d'erreurs, et Wayne et al. retrouvent une meilleure application des recommandations scientifiques après une formation sur simulateur $(110,146)$. Dans un autre domaine, les aptitudes en fibroscopie sont également améliorées significativement (durée d'intubation et taux de réussite) (147). Barsuk et al. retrouvent à la fois de graves lacunes dans la prise en charge du traumatisé crânien grave et du traumatisé thoracique, mais surtout une amélioration significative des compétences après une formation sur simulateur (148). Enfin pour Schwid et al., la formation par la simulation améliore considérablement la restitution des 
procédures médicales (149). Plusieurs articles, cités dans l'American Journal of Medical Quality (150), montrent que la simulation améliore les compétences des étudiants en médecine, des résidents et des professionnels dans le domaine de l'obstétrique. L'étude révèle que les participants ayant été formés par la simulation pratiquent plus de délivrances et ont un niveau de confiance supérieur en ce qui concerne les situations difficiles (par exemple face à des présentations compliquées).

La simulation médicale renforce donc les connaissances grâce à l'expérience sur le terrain, mais surtout elle va permettre de comprendre les barrières dans leur mise en œuvre (151).

Une méta-analyse récente et complète, ayant pour but de comparer les résultats de la formation par simulation par rapport à la formation " classique » (152), apporte des conclusions très intéressantes. Reprenant 10903 articles de la littérature, les auteurs ont sélectionné 609 études comportant 35226 participants. La formation par la simulation est constamment associée à une amélioration significative des connaissances, des pratiques et des comportements. En revanche, les effets sur la prise en charge des patients demeurent a priori modérés.

\subsection{Limites de la simulation}

Si la simulation présente de nombreuses qualités pour la formation, elle souffre cependant de quelques inconvénients et limites. La principale limite est le coût important pour la mise en place d'un programme de simulation. Celui-ci va se repartir sur les locaux, le matériel, mais surtout sur le personnel. Bien que l'utilisation du simulateur sur les lieux même de l'exercice soit préférable, des locaux dédiés sont le plus souvent nécessaires pour pouvoir organiser de façon régulière des séances et pour disposer d'une infrastructure audio-vidéo.

La présence d'un personnel qualifié en simulation semble indispensable (153). L'équipe de base pourra être représentée par un médecin, un infirmier et un technicien. Les quelques articles citant les ressources nécessaires montrent bien l'hétérogénéité des fonds utilisés. Selon les locaux (sur plans ou réhabilités), le matériel (matériel procédural ou mannequin HF), et le personnel présent, les frais de fonctionnement peuvent aller d'une dizaine de milliers à plus d'un million d'euros, avec parfois un coût de maintenance annuel qui peut presque représenter la moitié du coût d'investissement initial. En 1996, le coût de mise en place du Sunnybrook Health Center de Toronto était estimé à 665000 dollars US, dont $85 \%$ étaient destinés à l'achat du matériel et $15 \%$ aux salaires du personnel. Le coût annuel était de 165000 dollars US, essentiellement pour les salaires (78).

McIntosh a calculé, en 2007, que le coût initial était de 876485 dollars US pour la rénovation de locaux et l'achat du matériel, et 361425 dollars US pour le coût annuel de fonctionnement (154).

L'aspect émotionnel et stressant du simulateur constitue aussi un frein pour les participants. Le briefing joue ici un rôle capital pour rassurer les participants sur le caractère non sanctionnant de la formation qui leur est proposée. Leur nombre doit rester suffisamment limité, et c'est là une des contraintes face à l'apprentissage de masse des cours théoriques. Cela apporte en revanche une atmosphère propice à l'échange, mais aussi facilite la mise en situation (155).

Le debriefing est une étape essentielle d'une séance de simulation. Bien réalisée, elle doit surtout permettre une autoanalyse des participants. Elle doit faire envisager une " remise en cause ", la mise en œuvre d'actions d'amélioration et de perfectionnement, tant dans les aspects techniques que comportementaux.

Enfin, malgré les progrès croissants de la robotique, le matériel actuellement commercialisé n'arrive pas encore à recréer le réalisme d'un patient de chair et d'os. Toute la sémiologie cutanée (marbrure, éruption cutanée, cyanose, etc.) et la motricité, couramment utilisée pour évaluer le score de Glasgow, sont quasi inexistantes sur les mannequins actuels. L'avenir de la simulation passera alors probablement par la réalité virtuelle ou augmentée, déjà utilisée en chirurgie, pour s'amender de ses limites et proposer une simulation 
encore plus immersive aux participants (156). La recherche actuelle en simulation comporte encore de nombreuses insuffisances (travaux monocentriques, faibles échantillons, manque de rigueur statistique, etc.). Quelques travaux récents insistent sur l'importance d'une méthodologie rigoureuse pour la réalisation de projets multicentriques (41).

La simulation haute-fidélité constitue donc un outil de formation complet pouvant réduire le fossé entre une faible exposition aux situations critiques et une pratique répétée nécessaire à une prise en charge efficace. Malgré des difficultés, d'ordre essentiellement économique, la simulation est une méthode pédagogique d'intérêt. Elle est très largement acceptée et souhaitée, tant par les étudiants que les enseignants. Elle a déjà fait ses preuves dans différents domaines. Elle demande certainement à être développée, dans l'objectif prioritaire d'une amélioration des pratiques et de la gestion optimale des risques.

C'est pour l'ensemble de ces raisons que ce rapport propose dix actions qui permettraient, sans doute, un développement favorable de la simulation et un impact incontestable sur la qualité, la sécurité des soins.

\section{Simulation en santé : 10 propositions}

\section{Proposition 1}

$\rightarrow$ La formation par les méthodes de simulation en santé doit être intégrée dans tous les programmes d'enseignement des professionnels de santé à toutes les étapes de leur cursus (initial et continu). Un objectif éthique devrait être prioritaire : " jamais la première fois sur le patient".

\section{Proposition 2}

$\rightarrow$ L'importance de l'impact de la formation par la simulation sur les facteurs humains et le travail en équipe ainsi que son utilité dans la sécurité des soins doivent être largement étudiés.

\section{Proposition 3}

$\rightarrow$ Une politique nationale doit permettre à la formation par la simulation d'être valorisée et dotée de manière adaptée.

\section{Proposition 4}

$\rightarrow$ La formation initiale et continue par la simulation doit faire l'objet de coopérations entre les universités et les structures de soins ou les instituts de formation (publics ou privés).

\section{Proposition 5}

$\rightarrow$ Les formateurs en matière de simulation doivent bénéficier d'une compétence réelle, validée par l'obtention de diplômes universitaires spécifiques.

\section{Proposition 6}

$\rightarrow$ Chaque société savante doit identifier des programmes de formation par la simulation adaptés aux priorités de leur discipline.

\section{Proposition 7}

$\rightarrow$ L'ensemble des ressources doit faire l'objet d'une mutualisation selon des critères validés (plates-formes équipées accessibles, banque de scénario, programmes de DPC, etc.)

\section{Proposition 8}

$\rightarrow$ Au niveau national ou régional, les accidents les plus graves ou les plus signifiants doivent faire l'objet de reconstitutions en simulation afin d'en analyser les causes et de prévenir leur répétition.

\section{Proposition 9}

$\rightarrow$ La simulation peut être utilisée comme un outil de validation des compétences (ou de transfert de compétences) des professionnels au sein de structures « certifiées ».

\section{Proposition 10}

$\rightarrow$ Les travaux de recherche sur la simulation en santé doivent faire l'objet d'une méthodologie rigoureuse et d'une collaboration en réseau. 


\section{Annexe 1. Arrêté du 30 juin 1999 relatif à l'utilisation des simulateurs dans les formations conduisant à la délivrance de titres de formation professionnelle maritime}

Tout simulateur utilisé dans une formation conduisant à la délivrance d'un titre de formation professionnelle maritime doit :

- être adapté aux objectifs fixés et aux tâches à effectuer dans le cadre de la formation ;

- être capable de simuler les capacités de fonctionnement du matériel de bord concerné, avec un réalisme d'un niveau approprié aux objectifs de formation, et incorporer les capacités, limitations et erreurs possibles de ce matériel ;

- simuler le comportement avec suffisamment de réalisme afin de permettre à un élève d'acquérir les aptitudes correspondant aux objectifs de formation ;

- créer un environnement d'exploitation contrôlé, capable de produire diverses conditions qui peuvent inclure des situations d'urgence, des situations potentiellement dangereuses ou des situations inhabituelles en rapport avec les objectifs de formation ;

- fournir une interface permettant une interaction entre l'élève et le matériel, l'environnement simulé et, selon le cas, l'instructeur ;

- permettre à l'instructeur de contrôler, de surveiller et d'enregistrer les exercices, afin qu'il puisse analyser efficacement avec l'élève sa prestation.

Tout simulateur, utilisé pour évaluer les compétences requises ou pour toute démonstration du maintien des compétences, doit :

- être capable de répondre aux objectifs d'évaluation spécifiés ;

- être capable de simuler les capacités de fonctionnement du matériel de bord concerné, avec un réalisme d'un niveau approprié aux objectifs d'évaluation, et incorporer les capacités, limitations et erreurs possibles de ce matériel ;

- simuler le comportement avec suffisamment de réalisme, afin de permettre au candidat de montrer les aptitudes correspondant aux objectifs d'évaluation ;

- fournir une interface permettant une interaction entre le candidat, le matériel et l'environnement simulé ;

- créer un environnement d'exploitation contrôlé, capable de produire diverses conditions qui peuvent inclure des situations d'urgence, des situations potentiellement dangereuses ou des situations inhabituelles en rapport avec les objectifs d'évaluation ;

- permettre à l'évaluateur de contrôler, de surveiller et d'enregistrer les exercices, afin qu'il puisse évaluer de manière efficace les prestations des candidats. 


\section{Annexe 2. Textes réglementaires}

$\rightarrow$ Loi du 09 août 2004 portant sur l'obligation de FMC.

$\rightarrow$ Loi du 13 Août 2004 relative à l'assurance maladie :

- (art. 14 faisant obligation d'EPP individuelle) ;

- (art. 16 sur l'accréditation volontaire des médecins).

$\rightarrow$ Décret du 14 avril 2005 relatif à l'EPP.

$\rightarrow$ Ordonnance $n^{\circ} 2005-406$ du 02 mai 2005.

$\rightarrow$ Certification des établissements de santé version 2 (réf. 40, 41 et 42).

$\rightarrow$ Décrets $n^{\circ}$ 2006-650 du 2 juin 2006 modifiant la 4e partie du CSP (médecins).

$\rightarrow$ Décrets n² 2006-651 du 2 juin 2006 (pharmaciens).

$\rightarrow$ Décrets $n^{\circ} 2006-652$ du 3 juin 2006 (odontologistes).

$\rightarrow$ Arrêté du 13 juillet 2006 : homologation des règles de validation de la FMC.

$\rightarrow$ Décision $n^{\circ}$ 2007.10.035/EPP du 7 novembre 2007, relative aux modalités de mise en œuvre de l'évaluation des pratiques professionnelles.

$\rightarrow$ Décision $n^{\circ}$ 2007.12.042/EPP du 19 décembre 2007, modifiant la décision 2007.10.035/EPP du 7 novembre 2007 de la HAS relative aux modalités de mise en œuvre de l'EPP.

$\rightarrow$ Loi du 21 juillet 2009 ( $n^{\circ}$ 2009-879) dite « Loi Hôpital Patients Santé Territoires » ou HPST. Article 59 relatif au développement professionnel continu (DPC).

$\rightarrow$ Manuel de certification V2010, version avril 2011.

$\rightarrow$ Décret $n^{\circ}$ 2011-2113 du 30 décembre 2011 relatif à l'organisme gestionnaire du développement professionnel continu.

$\rightarrow$ Décret $n^{\circ}$ 2011-2115 du 30 décembre 2011 relatif au développement professionnel continu des chirurgiens-dentistes.

$\rightarrow$ Décret $n^{\circ}$ 2011-2116 du 30 décembre 2011 relatif au développement professionnel continu des médecins.

$\rightarrow$ Décret $n^{\circ}$ 2011-2114 du 30 décembre 2011 relatif au développement professionnel continu des professionnels de santé paramédicaux.

$\rightarrow$ Décret $n^{\circ}$ 2011-2118 du 30 décembre 2011 relatif au développement professionnel continu des pharmaciens.

$\rightarrow$ Décret $n^{\circ}$ 2011-2117 du 30 décembre 2011 relatif au développement professionnel continu des sagesfemmes. 


\section{Annexe 3. Plaquette décrivant brièvement les différentes méthodes de simulation jointe au questionnaire d'enquête}

\section{Qu'entend-on par "Simulation » en Santé?}

Pour parler de formation par la simulation il faut:

- Une mise en situation simulée d'un professionne ou d'une équipe

- Selon un protocole préétabli (scénario)

- Un débriefing à l'issue de la séance avec les participants et les formateurs (soit immédiat soit différé)

- Du matériel spécifique peut être utilisé

(exemples de mannequins représentant le patient) - Un enregistrement vidéo peut ou non être réalisé - La séance peut concerner des professionnels de santé en formation initiale ou continue
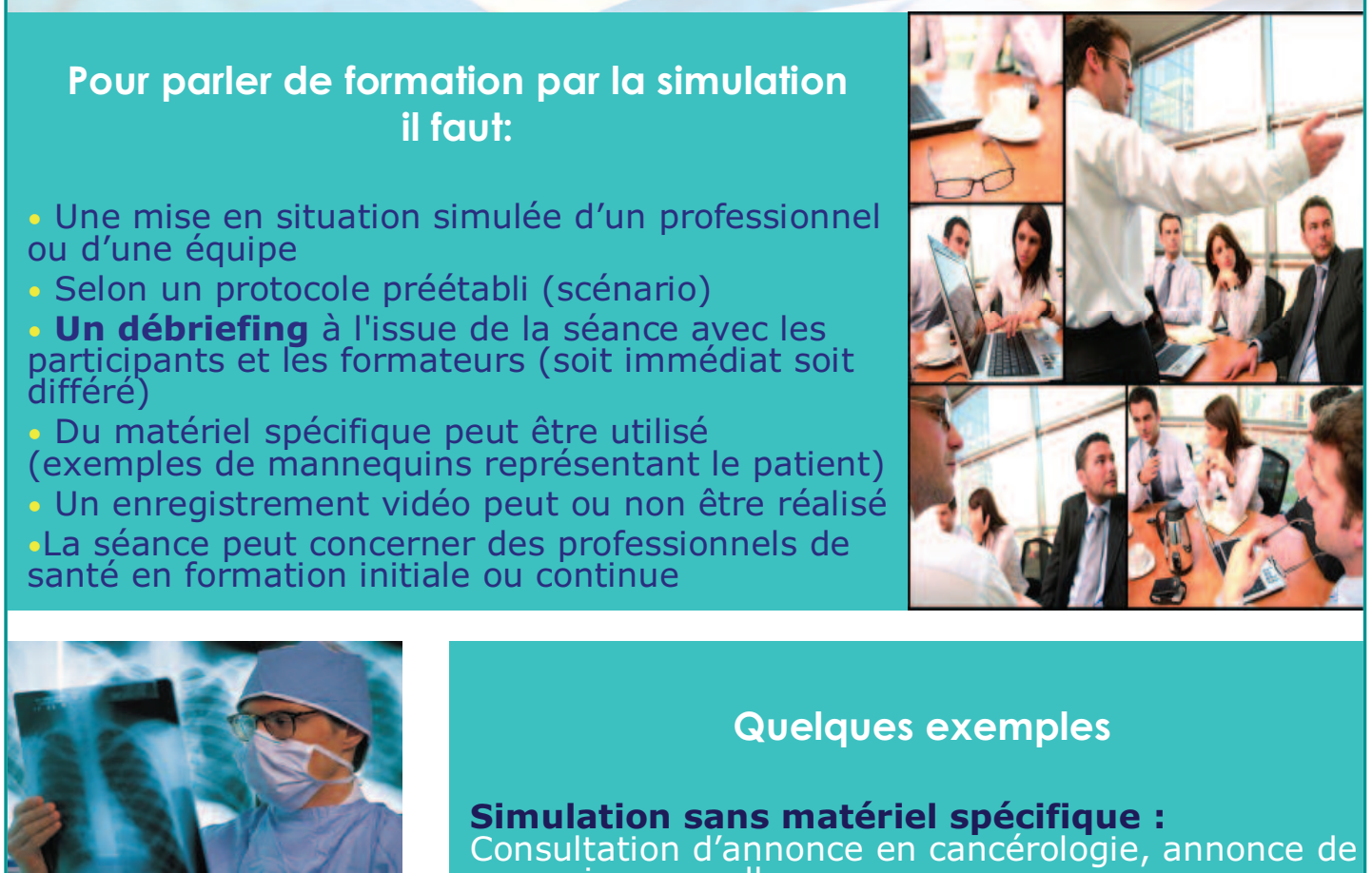

\section{Quelques exemples}

Simulation sans matériel spécifique :

Consultation d'annonce en cancérologie, annonce de mauvaise nouvelle

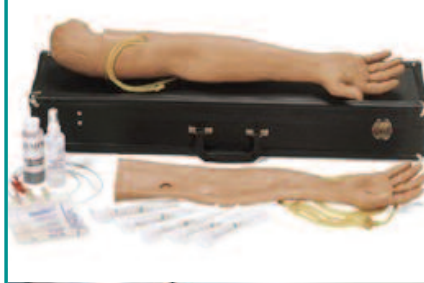

Simulation procédurale, dite basse fidélité :

Bras pour pose d'une voix veineuse, torse pour cathétérisme sous clavier, tête pour intubation

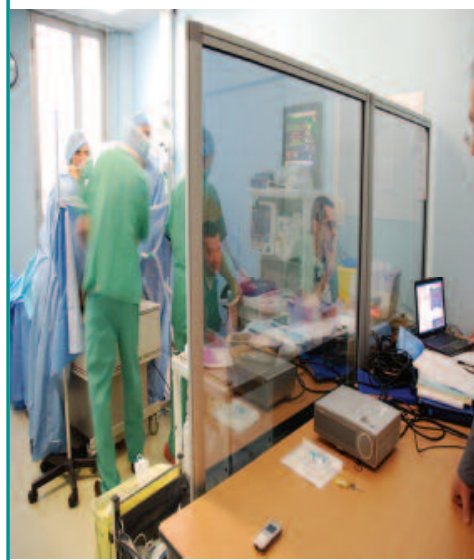

\section{Simulation dite haute fidélité:}

Mannequin copiant le vivant, piloté par ordinateur par exemple pour les situations d'urgence en environnement de bloc opératoire

\section{Autre:}

-Animation de réunion

-Simulation d'entretien pour auditeur

Simulation des plans de secours.

Version du 02/11. MCM . CHU Angers 


\section{Annexe 4. Exemple de programme d'EPP par la simulation en cancérologie dans le cadre du DPC}

\section{Consultation d'annonce du cancer}

\begin{tabular}{|l|l|}
\hline Thématique : CANCER & Code \\
\hline Structure DPC : Centre de simulation D'ANGERS & \\
Pilotage du projet : à compléter & \\
Équipe pédagogique : à compléter & \\
Méthodologie DPC : à compléter & \\
\hline
\end{tabular}

\section{Partie 1. Construction du programme d'EPP (Réservée à la structure organisatrice)}

\section{Caractéristiques du programme d'EPP}

$\rightarrow$ Développer le niveau de performance des professionnels devant réaliser la consultation d'annonce conformément au Plan cancer, améliorer les modalités d'information des patients atteints de cancer.

$\rightarrow$ Développer des habiletés sur des situations progressivement complexifiées.

\subsection{Cadre}

Dans le cadre du développement professionnel continu, un programme d'évaluation des pratiques professionnelles concernant l'amélioration des pratiques de consultation d'annonce du cancer est défini.

\subsection{Validation}

Ce programme est validé par la commission EPP de la CME, en ce qu'elle constituera à terme un organisme agréé de DPC (décret à paraître). Ce programme peut être validé par un organisme agréé en cancérologie (INCA ?).

\subsection{Engagement}

Les professionnels, souhaitant obtenir la validation de leur EPP, s'engagent à suivre l'ensemble du programme par :

- le suivi de l'exhaustivité des séances prévues ;

- le suivi des propositions qui seront faites en matière d'amélioration de leurs pratiques à l'issue du programme ;

- la participation à l'étude d'impact.

Le respect de cet engagement conditionne l'attribution du certificat de DPC. 


\subsection{Déontologie}

Le programme est réalisé dans les règles déontologiques de confidentialité inhérente à toute évaluation professionnelle individuelle.

La réalisation des méthodes de simulation est assurée par un centre ayant une compétence reconnue dans ce domaine.

Compte tenu de la nature des scénarios, un psychologue est sollicité pour l'accompagnement des professionnels.

\subsection{Public concerné}

Les médecins, internes, les IDE et tout professionnel pouvant potentiellement participer à une consultation d'annonce de cancer.

\subsection{Durée du programme}

Deux années afin d'intégrer la mesure d'impact.

\section{Nature de l'outil d'EPP}

\subsection{Outil d'évaluation de la pratique}

$\rightarrow$ L'outil utilisé est la simulation de la situation clinique avec patient standardisé.

$\rightarrow$ Le scénario est bâti sur la base des bonnes pratiques citées ci-dessous.

$\rightarrow$ Le scénario est suivi d'un debriefing permettant aux participants une autocritique.

$\rightarrow$ Les bonnes pratiques seront rappelées.

$\rightarrow$ Un compte-rendu écrit d'évaluation est rendu aux participants et comporte les conseils en vue de l'amélioration des pratiques.

\subsection{Outil d'évaluation d'impact du programme}

Cette évaluation du programme est réalisée selon trois axes :

- la satisfaction des professionnels formés ;

- l'impact sur la pratique du professionnel ;

- l'impact sur la qualité de la prise en charge du patient (satisfaction du patient).

\section{Validité du programme}

Pour répondre au critère d'EPP, le programme réalisé repose sur des référentiels de pratique :

- « Information des patients, recommandations destinées aux médecins » - Anaes - Mars 2000.

- «Les recommandations nationales pour la mise en œuvre du dispositif d'annonce du cancer dans les établissements de santé »- Novembre 2005.

Il inclut des actions d'amélioration de la pratique mesurables. 


\section{Construction du programme}

Le programme se déroule selon le plan suivant (les séances pourront être espacées de un à trois mois).

\section{Principe de la séance}

$\rightarrow$ Accueil des participants au centre de simulation.

$\rightarrow$ Présentation par l'équipe du centre de simulation du principe de la formation en simulation :

- du principe de la consultation d'annonce (réglementation, directives INCA) ;

- briefing des participants sur leur intervention.

$\rightarrow$ Réalisation du scénario de la consultation d'annonce en simulation.

$\rightarrow$ Séance filmée puis débriefée.

$\rightarrow$ Debriefing avec les participants et l'équipe 3C, en particulier avec l'aide des psychologues.

$\rightarrow$ Compte-rendu écrit global pour les participants.

\section{Séance 1}

Difficulté niveau 1 (par exemple, un patient seul, situation simple).

\section{Séance 2}

Difficulté 2 (par exemple, un patient et un ou deux membres de sa famille, situation incurable pathologie simple).

\section{Séance 3}

Difficulté niveau 3 (patient difficile, pathologie complexe).

Réalisation des préconisations aux participants

Évaluation de l'impact

Évaluation intermédiaire ; évaluation finale.

\section{Confidentialité}

Toutes les données de l'évaluation personnelles sont confidentielles.

Les images, réalisées lors de la simulation, ne seront pas diffusées ou utilisées sans autorisation expresse des participants.

Signature du document de restriction de l'utilisation de l'image en annexe.

Scénario 1 « Consultation d'annonce d'un cancer niveau de difficulté 1 »

Un cancer bronchique vient d'être diagnostiqué chez M. A. 63 ans.

Vous êtes son médecin et vous allez aujourd'hui lui donner les résultats du bilan que vous avez demandé après la mise en évidence d'une anomalie sur la radiographie thoracique.

M. A. est ancien plombier, en retraite depuis 2 ans. II a fumé pendant 30 ans un paquet de cigarettes par jour. II a arrêté depuis votre première consultation. II sait qu'il a manipulé des produits renfermant de l'amiante.

Il est accompagné de son épouse. 
Le bilan que vous avez réalisé identifie :

- une tumeur maligne du lobe supérieur droit ;

- le bilan d'extension est négatif ;

- la fibroscopie bronchique est normale ;

- le bilan d'opérabilité ne révèle pas de contre-indication opératoire.

Vous devez lui annoncer, ce matin, qu'il a un cancer et qu'une opération à visée curatrice peut lui être proposée. Vous devez lui expliquer que des traitements complémentaires peuvent lui être proposés selon les résultats des analyses postopératoires. Vous devez aussi obtenir son accord en vue de présenter son dossier en réunion de concertation pluridisciplinaire. Le patient vous attend dans la salle d'attente.

Faire sonner un téléphone pendant la consultation.

Scénario 2 « Consultation d'annonce d'un cancer niveau de difficulté 2 »

Scénario 3 « Consultation d'annonce d'un cancer niveau de difficulté 3 » 


\section{Annexe $4 b$. Annonce d'un décès à une famille}

\section{Temps estimé : 20 minutes}

\begin{tabular}{|c|c|c|c|}
\hline Algorithme & Scénario & Objectifs pédagogiques & \\
\hline $\begin{array}{l}\text { Centre hospitalier périphérique } \\
\text { sur une garde de nuit à } 22 \\
\text { heures : homme de } 84 \text { ans } \\
\text { adressé au déchoquage après } \\
\text { un AVP piéton contre VL. Le } \\
\text { patient aurait traversé par } \\
\text { mégarde. } \\
\text { Atcd : angine poitrine, ACFA, } \\
\text { Alzheimer, HTA. } \\
\text { Traitement : PREVISCAN, } \\
\text { TANAKAN } \\
\text { ACR sur place récupéré } \\
\text { initialement par l'équipe du } \\
\text { SAMU + ACR durant le } \\
\text { transport. Nouvel ACR au } \\
\text { déchoquage: } 30 \text { minutes de } \\
\text { réanimation. Décision collégiale } \\
\text { d'arrêt de réanimation (réflexion } \\
\text { entre SAMU et médecin de } \\
\text { garde). } \\
\text { La famille a suivi, est avertie par } \\
\text { des voisins. Ils ne sont au }\end{array}$ & $\begin{array}{l}\text { La famille a été envoyée en salle } \\
\text { d'attente du déchoquage. } \\
\text { Infirmière appelée en salle } \\
\text { d'attente, car " petit ॥ malaise } \\
\text { d'un membre de la famille. Ils } \\
\text { s'impatientent, tentent d'avoir des } \\
\text { nouvelles. } \\
\text { Le médecin n'arrive qu'ensuite. } \\
\text { Phrases à prononcer par la } \\
\text { famille: } \\
\text { - quelles seront les séquelles? } \\
\text { - qui est responsable de } \\
\text { l'accident? } \\
\text { - pompiers arrivé trop tard? } \\
\text { - il aurait souhaité donner ses } \\
\text { organes? } \\
\text { - qu'allons-nous faire maintenant? }\end{array}$ & $\begin{array}{l}\text { 1. Accueil de la famille } \\
\text { 2. Annonce de diagnostic } \\
\text { grave } \\
\text { 3. Bien se faire comprendre } \\
\text { et s'assurer d'avoir bien } \\
\text { été compris } \\
\text { 4. Proposer soutien }\end{array}$ & $\begin{array}{l}\text { Matériels : } \\
\text { - table salle d'attente ; } \\
\text { - chaises ; } \\
\text { - carafe d'eau + verres ; } \\
\text { - blouse médecin et IDE. } \\
\text { Acteurs : } \\
\text { Selon effectifs encadrants, } \\
\text { idéalement non connus des } \\
\text { participants, } 2 \text { à } 3 \text { membres } \\
\text { de la famille: } \\
\text { - mutiques ; } \\
\text { - agressifs ; } \\
\text { - ne comprend rien. } \\
\text { Public cible : } \\
\text { - jeune senior ; } \\
\text { - interne avancé dans son } \\
\text { cursus ; } \\
\text { - infirmière de réanimation; } \\
\text { - IADE. }\end{array}$ \\
\hline courant de rien. & & $\begin{array}{l}\text { Alternative : scénario } \\
\text { demande PMO }\end{array}$ & \\
\hline
\end{tabular}




\section{Annexe 5. Exemple de programme d'EPP par la simulation dans le cadre du DPC}

\section{Mise en œuvre de la check-list opératoire}

\begin{tabular}{|l|l|}
\hline $\begin{array}{l}\text { Thématique du programme de DPC : sécurité } \\
\text { au bloc opératoire }\end{array}$ & Code \\
\hline $\begin{array}{l}\text { Structure DPC :Centre de simulation xxx } \\
\text { Pilotage du projet : à compléter }\end{array}$ & \\
Équipe pédagogique : à compléter & \\
Méthodologie DPC : à compléter & \\
\hline
\end{tabular}

\section{Partie 1. Construction du programme d'EPP dans le cadre du DPC (Réservé à la structure organisatrice)}

\section{Caractéristiques du programme}

\subsection{Objectifs du programme d'EPP}

Entraîner les équipes médicales et paramédicales de bloc opératoire à la bonne utilisation de la check-list, en vue de sécuriser la prise en charge des patients opérés. Les scénarios concerneront les équipes de bloc opératoire pour les interventions programmées, l'ambulatoire et les actes invasifs nécessitant l'utilisation de la check-list.

Développer les synergies d'équipe autour de la check-list pour en assurer le bon usage, le cycle de formation abordera des situations progressivement complexifiées.

\subsection{Cadre}

Dans le cadre du développement professionnel continu, le programme d'évaluation des pratiques professionnelles concerne l'amélioration des pratiques pré, per et postopératoires.

\subsection{Validation}

Ce programme est validé par la commission EPP de la CME, en ce qu'elle constituera à terme un organisme agréé de DPC (décret à paraître). Ce programme peut être validé par un organisme agréé en chirurgie, en anesthésie-réanimation ou dans les disciplines spécifiques aux actes interventionnels.

\subsection{Engagement}

Les professionnels qui souhaitent obtenir la validation de leur EPP s'engagent à suivre l'ensemble du programme :

- suivi de l'exhaustivité des séances prévues ;

- suivi des propositions qui seront faites en matière d'amélioration de leurs pratiques à l'issue du programme ;

- participation à l'étude d'impact.

Le respect de cet engagement conditionne l'attribution du certificat de DPC. 


\subsection{Déontologie}

Le programme est réalisé dans les règles déontologiques de confidentialité inhérente à toute évaluation professionnelle individuelle.

La réalisation des méthodes de simulation est assurée par un centre ayant une compétence reconnue dans ce domaine (centre agréé).

Compte tenu de la nature des scénarios, un gestionnaire de risque doit être associé, de même un psychologue peut être sollicité pour l'accompagnement des professionnels.

Les vidéos, réalisées, ne seront utilisées que dans le strict cadre de la séance de simulation. Aucune diffusion publique ne sera autorisée.

\subsection{Public concerné}

Les médecins anesthésistes, les médecins opérateurs (chirurgiens, médecins réalisant des actes interventionnels) internes, les IDE, IADE et IBODE et tout professionnel pouvant potentiellement participer à la réalisation de la check-list.

\subsection{Durée du programme}

Deux années afin d'intégrer la mesure d'impact.

\section{Nature de l'outil d'EPP}

\subsection{Outil d'évaluation de la pratique}

$\rightarrow$ L'outil utilisé est la simulation de la situation clinique avec mannequin haute-fidélité ou patient simulé, contextualisé dans un environnement de bloc opératoire, de structure d'ambulatoire ou de réalisation d'acte interventionnel (endoscopie, imagerie interventionnelle, pose de cathéter implantable).

$\rightarrow$ Le scénario est bâti sur la base des bonnes pratiques citées ci-dessous.

$\rightarrow$ Le scénario est suivi d'un debriefing permettant aux participants, en particulier, une autocritique.

$\rightarrow$ Les bonnes pratiques seront rappelées.

$\rightarrow$ Un compte-rendu écrit d'évaluation est rendu aux participants et comporte les conseils en vue de l'amélioration des pratiques.

\subsection{Outil d'évaluation d'impact du programme}

Cette évaluation du programme est réalisée selon trois axes :

- évaluation du progrès sur la base du document de satisfaction des professionnels formés : questionnaire de satisfaction à l'issue de chaque séance et à la fin du programme ;

- impact sur la pratique du professionnel ;

- auto-questionnaire concernant l'impact sur la pratique à 6 mois après la dernière séance à partir de la fiche d'aide à la progression,

I audit de pratique au bloc ;

- impact sur la qualité de la prise en charge du patient :

I nombre de dysfonctionnements relevés par la check-list avant et après formation. 


\section{Validité du programme}

Pour répondre au critère d'EPP, le programme réalisé repose sur des référentiels de pratique :

- check-list « Sécurité du patient au bloc opératoire », document HAS, version 2011 - 01 ;

- check-list « Sécurité du patient en endoscopie digestive » (SFED/HAS 2011);

- check-list « Sécurité du patient en endoscopie bronchique » (FFP/HAS 2011) ;

- check-list « Pose d'un cathéter veineux central (CVC) ou autre dispositif vasculaire (DV) » ;

- revue bibliographique HAS, 2010. V11.1, mise à jour le 1er novembre 2010.

II inclut des actions d'amélioration de la pratique mesurables.

\section{Construction du programme}

Le programme se déroule selon le plan suivant (les séances pourront être espacées de un à trois mois).

\section{Principe de la séance}

$\rightarrow$ Accueil des participants au centre de simulation.

$\rightarrow$ Pré-briefing :

1 présentation par l'équipe du centre de simulation du principe de la formation en simulation ;

I du principe de la check-list ;

- information des participants sur leur intervention, prise de connaissance de l'histoire, du contexte, de l'environnement techniques (locaux, matériel support).

Réalisation du scénario de la situation interventionnelle. II est découpé en quatre séquences indépendantes (pré avec patient standardisé ; per et post avec mannequin HF) :

- séance filmée ;

- debriefing avec les participants, l'équipe du centre de simulation, le gestionnaire de risques, le psychologue si besoin ;

- compte-rendu écrit global pour les participants ;

- remise du document d'aide à la progression.

\section{Séance 1}

Difficulté niveau 1 : objectif - observer comment l'équipe utilise la check-list pendant les phases pre, per et post-interventionnelle, situation simple d'utilisation de la check-list, comportant une erreur à repérer. La coopération des acteurs est observée. En ce qui concerne le modèle d'outil EPP, audit clinique, la comparaison de la pratique réalisée par rapport à la pratique préconisée est effectuée. Ici toute l'équipe est testée.

\section{Séance 2}

Difficulté niveau 2 : l'équipe passant en simulation est confrontée aux résistances à la mise en œuvre (dans ce cas des acteurs sont introduits dans le scénario). Les observations retenues sont les stratégies pour obtenir la compliance de l'équipe et la sécurité du patient. 


\section{Séance 3}

Difficulté niveau 3 : à la situation 2 sont ajoutées des anomalies relevées par la check-list. Ce qui est observée est la réaction de l'équipe vis-à-vis de la check-list et des actions correctives apportées aux dysfonctionnements relevés par celle-ci. Dans le debriefing, sera évoquée l'efficacité des check-list pour la sécurité du patient.

\section{Remarque}

Le scénario sera adapté en fonction de la cible : interventions programmées, ambulatoire ou actes interventionnels.

\section{Réalisation des préconisations aux participants}

Remise de la fiche de progression.

\section{Évaluation de l'impact}

Pour l'évaluation finale, on pourra également en proposer une intermédiaire.

\section{Confidentialité}

Toutes les données de l'évaluation personnelles sont confidentielles.

Les images réalisées, lors de la simulation, ne seront pas diffusées ou utilisée sans autorisation expresse des participants (signature du document de restriction de l'utilisation de l'image en annexe). 


\section{Annexe 5b. Scénario 1 - Mise en œuvre simple de la check- list au bloc opératoire : phases pré, per et post- opératoire}

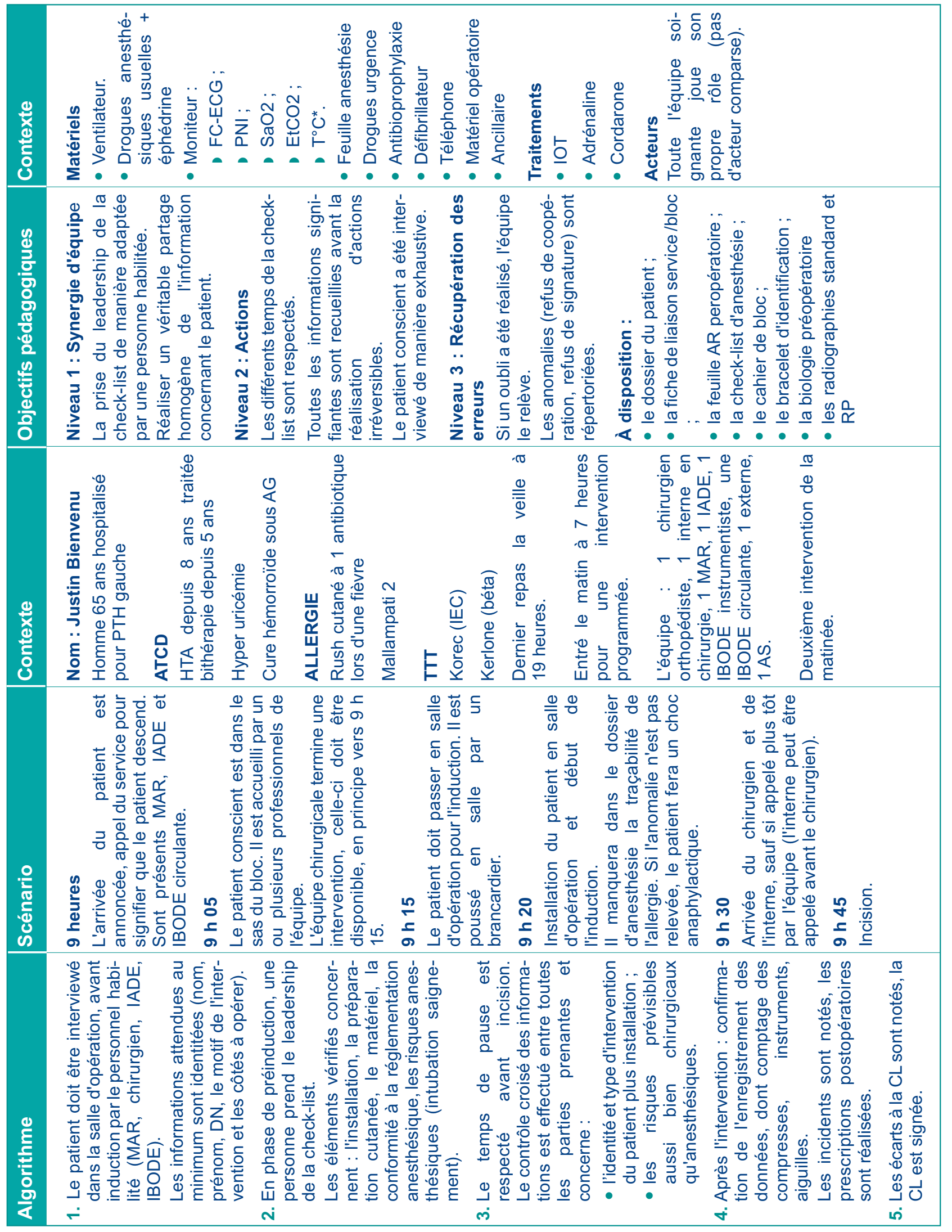




\section{Annexe 6. Deuxième partie de programme EPP justificatif DPC (Applicable aux deux exemples de programme d'EPP ci-dessus)}

Partie 2. Justificatif du programme (Remplie conjointement par le demandeur et la structure de DPC)

Médecin (s) inscrit (s) dans le programme

Nom :

Prénom :

Discipline :

Pôle :

1. Objectifs pédagogiques du programme

1.1 Objectifs institutionnels (à remplir par pôle, structure spécifique, 3C, CLIN, etc.)

1.2 Objectifs personnels (à remplir par le professionnel adhérent au programme)

1.3 Autres éléments du programme de DPC personnel

2. Recommandations de la structure de DPC (Remplies à l'issue du programme par le centre de formation)

3. Plan d'action (Rempli par le professionnel si besoin)

4. Résultats des mesures d'impact

\section{Validation}

\begin{tabular}{|l|l|l|}
\hline $\begin{array}{l}\text { Engagement du professionnel } \\
\text { inscrit dans le programme }\end{array}$ & Responsable de pôle & $\begin{array}{l}\text { Validation du programme par } \\
\text { organisme de DPC }\end{array}$ \\
\hline Nom, prénom & Nom, prénom & Nom, prénom \\
Visa & Visa & Visa \\
\hline
\end{tabular}




\section{Annexe 7. Questionnaire d'enquête complet}

\section{Grille d'évaluation des centres de simulation}

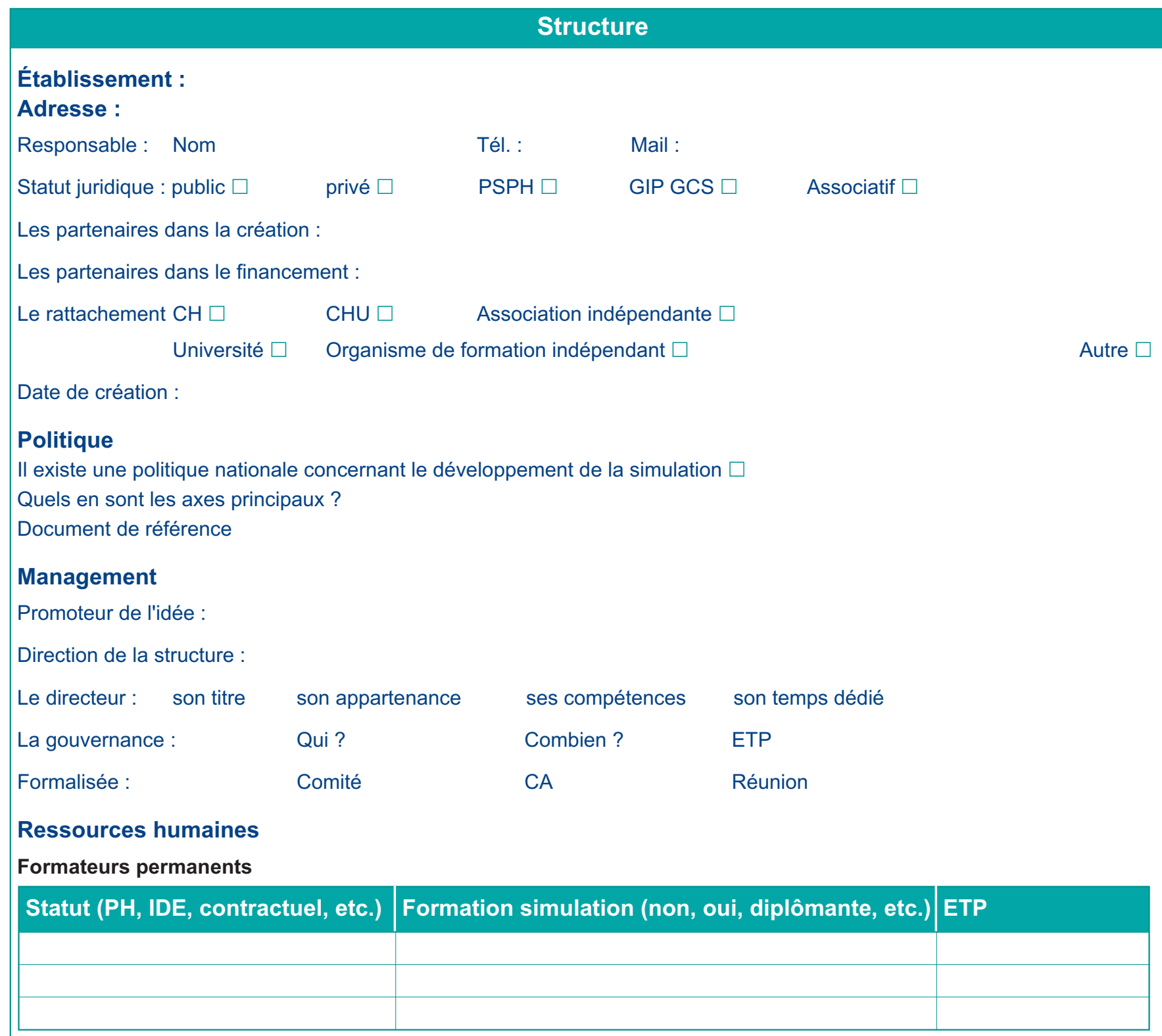

Formateurs intervenants ponctuels

\begin{tabular}{|l|l|l|}
\hline Statut (PH, IDE, contractuel, etc.) & Formation simulation (non, oui, diplômante, etc.) & ETP \\
\hline & & \\
\hline & & \\
\hline
\end{tabular}

Encadrement opérationnel

\begin{tabular}{|l|l|l|l|}
\hline Statut (PH, IDE, etc.) & Mission & ETP & Personnes \\
\hline & & & \\
\hline & & & \\
\hline & & & \\
\hline
\end{tabular}




\section{Ressources humaines (suite) \\ Personnels supports}

\begin{tabular}{|l|l|l|l|}
\hline Statut & Mission & ETP & Personnes \\
\hline Hôtesse & & & \\
\hline Secrétaire & & & \\
\hline Autre personnel administratif & & & \\
\hline Entretien des locaux & & & \\
\hline Maintenanace des équipements & & & \\
\hline Communication & & & \\
\hline Démarchage & & & \\
\hline Qualiticien & & & \\
\hline Autre personnel technique & & & \\
\hline
\end{tabular}

\section{Ressources matérielles : locaux (vous pouvez joindre un plan détaillé)}

1) Locaux dédiés exclusivement à la formation, par la simulation oui non (si non, précisez avec quelles activités le site est partagé)

Localisation des locaux :

2) Affectations et nombre des salles de simulation

Discipline ou thème surface : équipement vidéo

Discipline ou thème surface : équipement vidéo

Discipline ou thème surface : équipement vidéo

3) Affectation et nombre de locaux techniques

Salle de contrôle vidéo (répartition par salle de simulation) :

- 1 par salle de simulation 1 pour $n^{\circ}$ salle de simulation

- surface

Salle de debriefing :

- nombre :

- surface partagée avec une autre affectation : oui/non

Si oui, quoi ? : (exemple avec salle de pause)

Espace de convivialité :

- salle de pause $\quad \square \quad$ salle d'attente

- machine à café $\quad \square \quad$ zone d'accueil

- autre

Vestiaires:

Combien? $\quad$ Surface ?

- lavabos

$\square$

- douches

- sacs pour habillage

- capacité d'accueil simultané (nombre de personnes) :

Autres locaux :

Plan des locaux : (à joindre si possible) 


\section{Structure (suite)}

\section{Ressources matérielles : équipements}

En fonction des salles de simulation identifiées plus haut ,on listera par salle.

1. Type et caractéristiques du matériel vidéo de la salle

2. Type nombre et caractéristiques du matériel des salles de contrôle

3. Type caractéristique et nombre de matériel de simulation

- matériel procédural (bras, tête)

précisé :

- mannequin adulte HF

marque :

- mannequin enfant HF

marque :

- mannequin NN

marque :

- automate

précisé :

- réalité virtuelles

précisé :

- autre

4. Équipements lourds spécifiques

fluides $\square \quad$ table opératoire $\square$

environnement spécifique (chambre, domicile...)

précisé :

5. Consommables utilisés lors des simulations

nouveaux consommables à chaque séance

recyclage des consommables

\section{Modalités de financement}

\section{Financement initial}

Partenaire 1 :

niveau de financement :

Partenaire 2 :

niveau de financement :

Partenaire 3 :

niveau de financement :

Total financement de départ

Appréciation du niveau de difficulté pour obtenir le financement de départ

Très difficile

Difficile

Facile

Très facile

Délai pour réunir le financement?

Commentaire

\section{Déficit de financement}

Commentaire : à quoi devez-vous renoncer?

Tarif par personne des séances de simulation

Tarif groupe ou préférentiels :

Une séance correspond pour vous à 1 jour, 0,5 jour, autres ?

\section{Chiffre d'affaire annuel}

\section{Coûts}

1. Coût investissement :

- total équipement.

2. Coût de fonctionnent :

- total coût de maintenance annuel des matériels et équipement

- total charges personnel ;

- total charges indirectes (location);

- total du matériel. 


\section{Programme pédagogique}

Projet pédagogique :

rédigé

Si oui, quelles en sont les objectifs principaux ?

Contrats formalisés avec des organismes de formation professionnels

Si oui, lesquels?

FSI

réseaux

IFCS

Facultés de médecine

Autres organismes en santé

Faculté de pharmacie $\square$

Autres organismes hors santé

\section{Financement des programmes de formation}

- payant, pour qui ?

- gratuit, pour qui ?

- les deux, si oui proportion et pour qui ?

Formation initiale :

Si oui :

Interne médecine Interne pharmacie

Externe médecine Externe pharmacie

Élèves sage-femme

Élèves IDE (IADE, IBODE, puéricultrices, etc.)

Autre élèves paramédicaux

Autres stagiaires :

- \% selon le stade de la formation (début, milieu, fin) ;

- Total activité mensuelle en heures de formation homme ;

- Taux de remplissage de vos programmes de formation.

Formation continue :

Médecins séniors (disciplines)

Pharmaciens séniors

Sage-femme

IDE (IADE, IBODE, puér.)

Autre paramédicaux

Autres professionnels (pompiers, etc.)

- Moyenne d'âge des participants :

- Moyenne d'ancienneté dans l'activité :

- Sex ratio:

- Total activité mensuelle en heures de formation homme :

- Taux de remplissage de vos programmes de formation:

- Total activité mensuelle :

- Taux de progression sur 3 ans :
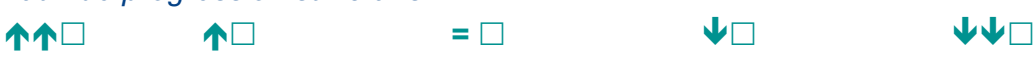

\section{Attractivité du centre}

locale

départementale $\square$

interrégionale $\square$

nationale

internationale $\square$

\section{Offre de formation}

1. Quels sont les champs de la formation ?

Amélioration des connaissances

Acquisition de connaissances

Amélioration des compétences

Acquisition de compétences

Gestion des risques associés aux soins

Habilitation de professionnels

Qualification des professionnels

Remise à niveau

Simulation de crise

Reconstitution d'accidents

Étude des facteurs humains

Développement professionnel 


\section{Programme pédagogique (suite)}

Offre de formation (suite)

2. Programmes de formation thématique

Si oui, lesquels ?

3. Sont-ils structurés selon des objectifs pédagogiques ?

Si oui, lesquels?

4. Comment faites-vous progresser votre offre de formation ?

en fonction des moyens dont vous disposez

en fonction des demandes qui vous sont adressée

en réalisant une évaluation prospective du marché

en recherchant les alliances avec d'autres disciplines

en saisissant des opportunités

en répondant à une contrainte forte (injonction de la structure dont vous dépendez)

en planifiant et en anticipant celle-ci sur le court moyen ou long terme

\section{Séance de simulation}

Quels sont les différents temps de votre programme ou séance de formation par la simulation ?

Avez-vous une banque de scénarios?

(préciser)

Quel est leur taux de renouvellement?

Plusieurs fois par an

Tous les ans

De temps en temps

Évaluez-vous vos séances de simulation?

si oui, comment?

- Questionnaire de satisfaction

- Impact sur la pratique

- Nombre de séquence par participant.

Délivrez-vous ?

- Une évaluation écrite de la séance

- Des conseils de formation pour progresse

\section{Avez-vous}

- Des programmes phare

- Les programmes rentables

- Des programmes innovants ou expérimentaux ou de recherche

Si oui, sont-ils financés votre revenu de formation?

- Bénéficiez-vous de financement spécifique recherche?

- Bénéficiez-vous d'un mécénat et de quel type?

\section{Communication}

Disposez-vous d'une politique de communication pour faire connaître vos activités ? Disposez-vous d'outils spécifiques de communication?

Site internet

Plaquette

Réseau de diffusion de l'information

(préciser)

8. Recherche

Avez-vous une activité de recherche?

Si oui :

- Laboratoire de recherche?

- Axes de recherche (préciser)?

- Programme en cours (préciser)?

- Publication (joindre bibliographie) ?

- Financement (préciser) ?

9. Comment évalueriez-vous votre notoriété ?

Pas connu

Rayonnement interrégiona
Connu dans ma ville

Rayonnement national
Rayonnement régional Rayonnement international 


\section{Annexe 8. Liste des contributeurs à cette étude}

Nous adressons nos remerciements les plus sincères aux professionnels rencontrés. Ils nous ont consacré du temps d'interview et/ou de visite au sein de leurs centres de simulation, et ont contribué à la rédaction de certains chapitres de cette étude.

$\rightarrow$ Guillaume Alinier, manager du programme de simulation, centre médical et de recherche Sidra, Doha, Qatar, professeur en éducation du personnel de santé par la simulation, université de Hertfordshire, Hatfield, Royaume-Uni (visite du centre de simulation).

$\rightarrow$ Pr René Amalberti, docteur en médecine et en psychologie, chercheur à l'IMASA-CERMA, chargé de mission auprès de la direction générale de l'aviation civile (depuis 1994), spécialiste en gestion des risques.

$\rightarrow$ Dr Jérôme Berton, médecin anesthésiste-réanimateur, responsable du centre de simulation du CHU d'Angers.

$\rightarrow$ Dr Sylvain Boet MD, MEd, Family Patient Simulation Center, St. Michael's Hospital, University of Toronto, Toronto, Ontario, Canada, Department of Anesthesiology.

$\rightarrow$ Jean-Pierre Clostermann, capitaine illimité, professeur de l'enseignement maritime et instructeur sur simulateur à l'ENSM du Havre (visite du centre de simulation de L'ENSM).

$\rightarrow$ Philippe Fauquet-Alekhine, consultant facteurs humains au CNPE de Chinon, chercheur au laboratoire de recherche pour les sciences de l'énergie) (visite du centre de simulation de la centrale nucléaire de Chinon).

$\rightarrow$ Pr Jean-Paul Fournier, responsable du centre de simulation de Nice et Pr Daniel Benchimol Doyen de la faculté de médecine de Nice (visite du centre de la faculté de médecine).

$\rightarrow$ Florence-Marie Jegoux, subdivision instruction, subdivision Nantes Atlantique-Ouest (visite du centre de simulation des aiguilleurs du ciel de l'aéroport Nantes Atlantique). 


\section{Annexe 9. Références bibliographiques}

1. Society for Simulation in Healthcare. Council for accreditation of healthcare simulation programs, accreditation standards and measurement criteria. Minneapolis: SSH; 2010.

http://ssih.org/uploads/committees/2011\%20Self\% 20Study\%20Tool.pdf

2. Issenberg SB, McGaghie WC, Petrusa ER, Lee GD, Scalese RJ. Features and uses of high-fidelity medical simulations that lead to effective learning: a BEME systematic review. Med Teach 2005;27(1):10-28.

3. DeMaria S, Levine AI, Bryson EO. The use of multi-modality simulation in the retraining of the physician for medical licensure. J Clin Anesth 2010;22(4):294-9.

4. Béguin $P$, Weill-Fassina $A$. La simulation en ergonomie: connaître, agir et interagir. Toulouse: Octares; 1997.

5. Pastré P. Apprendre par la simulation. De l'analyse du travail aux apprentissages professionnels. Toulouse: Octares; 2005.

6. Chiniara G. Simulation médicale pour acquisition des compétences en anesthésie. In: Société française d'anesthésie et de réanimation, ed. Congrès national d'anesthésie et de réanimation 2007. Conférences d'actualisation. Paris: SFAR; 2007. p. 41-9.

7. Cooper JB, Taqueti VR. A brief history of the development of mannequin simulators for clinical education and training. Postgrad Med J 2008;84(997):563-70.

8. Rosen KR. The history of medical simulation. J Crit Care 2008;23(2):157-66.

9. Rattner Gelbart N. The king's midwife. A history and mystery of madame du Coudray. Berkeley: University of California Press; 1998.

10. Chamberland G, Provost G. Jeu, simulation et jeu de rôle. Québec: Presse de l'Université du Québec; 1996.

11. Hoffman KI, Abrahamson S. The 'costeffectiveness' of Sim One. J Med Educ 1975;50(12 Pt1):1127-8.
12. Barrows HS. An overview of the uses of standardized patients for teaching and evaluating clinical skills. Acad Med 1993;68(6):443-51.

13. Institut of Medicine, Kohn LT, Corrigan JM, Donaldson MS. To err ris human. Bulding a safer health system. Washington: National Academy Press; 1999.

http://www.nap.edu/openbook.php?isbn=030906837 1

14. Clostermann JP. La conduite du navire marchand. Facteurs humains dans une activité à risques. Rennes: InfoMer; 2010.

15. Buessard MJ, Fauquet P. Impacts de la prescription sur les activités de travail en centrale nucléaire. Thème 3 : prescription efficacité santé. In: Société d'ergonomie de langue française, Evesque JM, Gautier AM, Revest C, Schwartz Y, Vayssiere JL, ed. 37ème congrès de la SELF "Les évolutions de la prescription". Paris: GREAT; 2002. p. 326-35.

16. Lagrange $\mathrm{V}$, Desmares $\mathrm{E}$. De l'erreur humaine au management de la sûreté, l'ergonomie est-elle encore légitime face aux nouveaux enjeux de la sûreté ? In: Société d'ergonomie de langue française, ed. 34ème Congrès de la SELF, Caen 15 septembre 1999. Toulouse: SELF; 1999.

17. Fauquet-Alekhine P. Facteurs Humains dans l'industrie nucléaire française. Rev Maritime 2010;(490):4-11.

18. Fauquet-Alekhine P. Analyse de risques des activités de travail en centrale nucléaire: du contexte de l'apprentissage à l'application. In: Société d'ergonomie de langue française, Vallery $G$, Amalberti $R$, ed. 38ème Congrès de la SELF, Paris "Modèles et pratiques de l'analyse du travail 1988-2003 15 ans d'évolution". Paris: SELF; 2003. p. 636-46.

19. Fauquet-Alekhine P. Importance of decentralized organization for safety sharing. In: European Federation of Chemical Engineering, Pasman HJ, Sharka J, Babinec F, ed. Loss Prevention and Safety Promotion in Process Industries. Vol A. Risk assessment safety management legislative and initiatives. Praha: 
PCHE; 2004. p. 1378-1380.

20. Klein D, Simoëns P, Theurier JP. Témoignage d'entreprise : une collaboration recherche-industrie conséquente sur l'utilisation pédagogique des simulateurs à EDF. In: Pastré $P$, ed. Apprendre par la simulation. De l'analyse du travail aux apprentissages professionnels. Toulouse: Octares; 2005.

21. Béguin P, Pastré P. Working, learning and designing through simulation. In: Bagnara S, Pozzi S, Rizzo A, Wright P, ed. 11th European conference on cognitive ergonomics: cognition, culture and design. Catalina, Italy. Rome: CNR Publishing; 2002. p. 5-13.

22. Pastré P. Apprendre par résolution de problème : le rôle de la simulation. In: Pastré $P$, ed. Apprendre par la simulation. De l'analyse du travail aux apprentissages professionnels. Toulouse: Octares; 2005. p. 17-40.

23. Fauquet-Alekhine P. Développement des pratiques de fiabilisation sur simulateur de pilotage de réacteur nucléaire. In: Association internationale des sociologues de langue française, ed. Colloque de l'Association internationale des sociologues de langue française. Risques industriels majeurs. Toulouse. AISLF: Toulouse; 2007.

24. Fauquet-Alekhine P. Cognitive disorder and professional development by training: comparison of simulator sessions for anaesthesists and for nuclear reactor pilots. In: European Society for Developmental Psychology, Harris P, Kagitcibasi C, ed. XVe Conference on developmental psychology, Bergen Norvège, 23-27 august 2011. Philadelphia: Thomson Reuters; 2011.

25. Fauquet-Alekhine P. Synthèse d'observations et d'analyses de l'activité de travail des instructeurs simulateurs. Note d'étude du CNPE de Chinon, référence: D.5170/DIR/NED/05.003. Chinon: CNPE Chinon; 2005.

26. Clot Y, Fernandez G, Carles L. Crossed selfconfrontation in the "clinic of activity". In: Bagnara S, Pozzi S, Rizzo A, Wright P, ed. 11th European conference on cognitive ergonomics: cognition, culture and design. Catalina, Italy. Rome: CNR Publishing; 2002. p. 13-8.

27. Clot Y. La fonction psychologique du travail.
Paris: PUF; 1999.

28. Fauquet-Alekhine P. Applied crossed confrontation for context evolution. In: Dey A, Kokinov B, Leake D, Turner R, Brézillon P, ed. 5th International and interdisciplinary conference on modeling and using context. Brevia and demonstrations presentations, Paris. CNPE Chinon: Chinon; 2005. p. 36-41.

29. Fauquet-Alekhine P. Confrontation croisée ou analyse collective sur la base de restitutions d'entretiens individuels : deux approches pour l'analyse évènementielle. Activités Rev Electro 2006;3(2):2-14.

30. Fauquet-Alekhine P, Pahuet N, Labrucherie M, Maridonneau C, Geeraerts T, Trabold F, et al. Améliorer la pratique professionnelle par la simulation. Toulouse: Octares; 2011.

31. Fauquet-Alekhine P. Use of simulator training for the study of operational communication. The case of pilots of French nuclear reactors : reinforcement of reliabilit. In: Society for Modeling \& Simulation International, ed. 43rd Annual simulation symposium proceedings. 11-15 April 2010, Orlando, Florida, USA. 2010 Spring simulation multiconference. Curran Associates Inc: Miami; 2010.

32. Le Bot P. Human reliability data, human error and accident models. Illustration through the Three Mile Island accident analysis. Reliab Engin System Safe 2004;83(2):153-67.

33. Fauquet-Alekhine P. Etude cognitive et impact organisationnel du voyant RGL. Note d'étude du CNPE de Chinon, référence: D.5170/DIR/NED/04.003. In: Chinon: CNPE Chinon; 2004.

34. Fauquet-Alekhine P. Phase expérimentale relative à la communication opérationnelle sécurisée. Résultats 2005. Note d'étude du CNPE de Chinon, référence: D.5170/DIR/NED/06.001. Chinon: CNPE de Chinon; 2006.

35. Soler L, Marescaux J. Simulation chirurgicale virtuelle : les premiers pas d'une nouvelle formation. In: Fauquet-Alekhine $\mathrm{P}$, Pahuet N, Labrucherie M, Maridonneau C, Geeraerts T, Trabold F, et al., ed. Améliorer la pratique professionnelle par la simulation. Toulouse: 
Octares; 2011

36. De La Garza C, Fadier E. Le retour d'expérience en tant que cadre théorique pour l'analyse de l'activité et de la conception sûre. Activités [rev électr] 2007;4(1):188-97.

\section{Fauquet-Alekhine P. Avantages et} inconvénients de l'avatar et du virtuel : analyse de la dimension psychologique sur simulateur pleine échelle, hybride, et simulateur de réalité virtuelle. In: Colloque serious games et simulation pour la gestion des risques, 4-5 october 2011. Vélizy: 2011.

38. Morgan PJ, Cleave-Hogg DM. Cost and resource implications of undergraduate simulatorbased education. Can J Anaesth 2001;48(8):827-8.

39. Andreatta PB, Woodrum DT, Birkmeyer JD, Yellamanchilli RK, Doherty GM, Gauger PG, et al. Laparoscopic skills are improved with LapMentor training: results of a randomized, double-blinded study. Ann Surg 2006;243(6):854-60.

40. Sutherland LM, Middleton PF, Anthony A, Hamdorf J, Cregan P, Scott D, et al. Surgical simulation: a systematic review. Ann Surg 2006;243(3):291-300.

41. Van Nortwick SS, Lendvay TS, Jensen AR, Wright AS, Horvath KD, Kim S. Methodologies for establishing validity in surgical simulation studies. Surgery 2010;147(5):622-30.

42. Cooper JB, Taqueti VR. A brief history of the development of mannequin simulators for clinical education and training. Qual Saf Health Care 2004;13(Suppl 1):i11-i18.

43. Abrahamson S, Denson JS, Wolf RM. Effectiveness of a simulator in training anesthesiology residents. $\mathrm{J}$ Med Educ 1969;44(6):515-9.

44. Gaba DM, DeAnda A. A comprehensive anesthesia simulation environment: re-creating the operating room for research and training. Anesthesiology 1988;69(3):387-94.

45. Chopra V, Engbers FH, Geerts MJ, Filet WR, Bovill JG, Spierdijk J. The Leiden anaesthesia simulator. Br J Anaesth 1994;73(3):287-92.

46. Gordon MS. Cardiology patient simulator. Development of an animated manikin to teach cardiovascular disease. Am J Cardiol 1974;34(3):350-5.

47. Calman L, Watson R, Norman I, Redfern S, Murrells T. Assessing practice of student nurses: methods, preparation of assessors and student views. J Adv Nurs 2002;38(5):516-23.

48. Alinier G, Hunt B, Gordon R, Harwood C. Effectiveness of intermediate-fidelity simulation training technology in undergraduate nursing education. J Adv Nurs 2006;54(3):359-69.

49. Nursing Midwifery Council. Simulation and practice learning project for pre-registration nursing programmes. Circular 38/2006. London: NMC; 2006.

50. Moule P, Wilford A, Sales R, Lockyer L. Student experiences and mentor views of the use of simulation for learning. Nurse Educ Today 2008;28(7):790-7.

51. Baillie L, Curzio J. Students' and facilitators' perceptions of simulation in practice learning. Nurse Educ Pract 2009;9(5):297-306.

52. Nursing Midwifery Council. Supporting direct care through simulated practice learning in the preregistration nursing programme. NMC Circular 36/2007. London: NMC; 2007.

http://www.nmc-uk.org/Documents/Circulars/2007 circulars/NMCcircular36_2007.pdf

53. Nehring WM. U.S. boards of nursing and the use of high-fidelity patient simulators in nursing education. J Prof Nurs 2008;24(2):109-17.

54. Rothgeb MK. Creating a nursing simulation laboratory: a literature review. J Nurs Educ 2008;47(11):489-94.

55. Messmer PR. Enhancing nurse-physician collaboration using pediatric simulation. J Contin Educ Nurs 2008;39(7):319-27.

56. Lassale V, Berton J, Bouhours G, Péres M, Bossard G, Granry JC, et al. Enquête européenne sur la pratique de la simulation médicale pédiatrique. Ann Fr Anesth Réanim 2009;28(78):628-33.

57. Reeves S, Freeth D, McCrorie P, Perry D. 'It teaches you what to expect in future': interprofessional learning on a training ward for 
medical, nursing, occupational therapy and physiotherapy students. Med Educ 2002;36(4): 337-44.

58. Ker J, Mole L, Bradley P. Early introduction to interprofessional learning: a simulated ward environment. Med Educ 2003;37(3):248-55.

59. Wisborg T, Castren M, Lippert A, Valsson F, Wallin CJ, Working Scandinavian Group. Training trauma teams in the Nordic countries: an overview and present status. Acta Anaesthesiol Scand 2005;49(7):1004-9.

60. Seropian M, Dillman D, Farris D. Statewide simulation systems: the next step for anesthesiology? Anesthesiol Clin 2007;25(2): 271-82.

61. Fey MK, Miltner RS. A competency-based orientation program for new graduate nurses. J Nurs Adm 2000;30(3):126-32.

62. Beyea SC, von Reyn LK, Slattery MJ. A nurse residency program for competency development using human patient simulation. J Nurses Staff Dev 2007;23(2):77-82.

63. Landry M, Oberleitner MG, Landry H, Borazjani JG. Education and practice collaboration: using simulation and virtual reality technology to assess continuing nurse competency in the long-term acute care setting. J Nurses Staff Dev 2006;22(4):163-9.

64. Bohan KJ. Human patient simulation in graduate anesthesia education [thesis]. Madison: The University of Wisconsin; 2007.

65. Alinier G. Nursing students' and lecturers' perspectives of objective structured clinical examination incorporating simulation. Nurse Educ Today 2003;23(6):419-26.

66. Parsh B. Characteristics of effective simulated clinical experience instructors: interviews with undergraduate nursing students. J Nurs Educ 2010;49(10):569-72.

67. Alinier G. A typology of educationally focused medical simulation tools. Med Teach 2007;29(8):e243-e250.

68. Nehring WM. Nursing simulation. A review of the past 40 years. Simul Gaming 2009;40(4):

\section{8-52.}

69. McCaughey CS, Traynor MK. The role of simulation in nurse education. Nurse Educ Today 2010;30(8):827-32.

70. Feldman LS, Sherman V, Fried GM. Using simulators to assess laparoscopic competence: ready for widespread use? Surgery 2004;135(1):28-42.

71. Gurusamy KS, Aggarwal R, Palanivelu L, Davidson BR. Virtual reality training for surgical trainees in laparoscopic surgery. Cochrane Database Syst Rev 2009; Issue 1:CD006575.

72. Brindley PG, Dunn WF. Simulation for clinical research trials: a theoretical outline. J Crit Care 2009;24(2):164-7.

73. Taekman JM, Hobbs G, Barber L, Phillips-Bute BG, Wright MC, Newman MF, et al. Preliminary report on the use of high-fidelity simulation in the training of study coordinators conducting a clinical research protocol. Anesth Analg 2004;99(2):521-7, table.

74. Girzadas DV, Antonis MS, Zerth H, Lambert M, Clay L, Bose S, et al. Hybrid simulation combining a high fidelity scenario with a pelvic ultrasound task trainer enhances the training and evaluation of endovaginal ultrasound skills. Acad Emerg Med 2009;16(5):429-35.

75. Boet S, Collange O, Mahoudeau G. La simulation hybride: un nouveau concept pour des nouveaux objectifs pedagogiques. Ann Fr Anesth Reanim 2010;29(5):407-8.

76. Flin R, Maran N. Identifying and training nontechnical skills for teams in acute medicine. Qual Saf Health Care 2004;13 Suppl 1:i80-i84.

77. Boet S, Bould MD, Bruppacher HR, Desjardins F, Chandra DB, Naik VN. Looking in the mirror: Selfdebriefing versus instructor debriefing for simulated crises. Crit Care Med 2011;39(6):1377-81.

78. Kurrek MM, Devitt JH. The cost for construction and operation of a simulation centre. Can J Anaesth 1997;44(11):1191-5.

79. Inventures. NHS Simulation provision and use study summary report. London: Department of 
Health; 2010.

80. Chief Medical Officer's. Safer medical practice. Machines, manikins and polomints. London: $\mathrm{CMO}$; 2008.

http://www.bmsc.co.uk/pdf/DH-096227.pdf

81. Blum MG, Powers TW, Sundaresan S. Bronchoscopy simulator effectively prepares junior residents to competently perform basic clinical bronchoscopy. Ann Thorac Surg 2004;78(1): 287-91.

82. Wayne DB, Butter J, Siddall VJ, Fudala MJ, Linquist LA, Feinglass $\mathrm{J}$, et al. Simulation-based training of internal medicine residents in advanced cardiac life support protocols: a randomized trial. Teach Learn Med 2005;17(3):210-6.

83. Wayne DB, Butter J, Siddall VJ, Fudala MJ, Wade LD, Feinglass $\mathrm{J}$, et al. Mastery learning of advanced cardiac life support skills by internal medicine residents using simulation technology and deliberate practice. J Gen Intern Med 2006;21(3):251-6.

84. Barsuk JH, McGaghie WC, Cohen ER, O'Leary KJ, Wayne DB. Simulation-based mastery learning reduces complications during central venous catheter insertion in a medical intensive care unit. Crit Care Med 2009;37(10):2697-701.

85. Barsuk JH, Cohen ER, Feinglass J, McGaghie WC, Wayne DB. Use of simulation-based education to reduce catheter-related bloodstream infections. Arch Intern Med 2009;169(15):1420-3.

86. Draycott TJ, Crofts JF, Ash JP, Wilson LV, Yard $\mathrm{E}$, Sibanda $\mathrm{T}$, et al. Improving neonatal outcome through practical shoulder dystocia training. Obstet Gynecol 2008;112(1):14-20.

87. Mayo PH, Hackney JE, Mueck JT, Ribaudo V, Schneider RF. Achieving house staff competence in emergency airway management: results of a teaching program using a computerized patient simulator. Crit Care Med 2004;32(12):2422-7.

88. Bruppacher HR, Alam SK, LeBlanc VR, Latter D, Naik VN, Savoldelli GL, et al. Simulation-based training improves physicians' performance in patient care in high-stakes clinical setting of cardiac surgery. Anesthesiology 2010;112(4):985-92.

89. Rodriguez-Paz JM, Mark LJ, Herzer KR, Michelson JD, Grogan KL, Herman J, et al. A novel process for introducing a new intraoperative program: a multidisciplinary paradigm for mitigating hazards and improving patient safety. Anesth Analg 2009;108(1):202-10.

90. Draycott T, Sibanda T, Owen L, Akande V, Winter $C$, Reading $S$, et al. Does training in obstetric emergencies improve neonatal outcome? BJOG 2006;113(2):177-82.

91. Huwendiek S, De leng BA, Zary N, Fischer MR, Ruiz JG, Ellaway R. Towards a typology of virtual patients. Med Teach 2009;31(8):743-8.

92. Meller G. A typology of simulators for medical education. J Digit Imaging 1997;10(3 Suppl 1): 194-6.

93. Sfez $M$, de Marcellis-Warin N, Pourreau A, Triadou $P$, Courault $M$, Lienhart $A$. Combiner entretiens et cas simulé pour identifier les facteurs favorisant la récupération. Risque Qual Milieu Soins 2008;5(3):141-50.

94. Vidal-Gomel C, Fauquet-Alekhine P, Guibert S. Réflexions et apports théoriques sur la pratique des formateurs et de la simulation. In: Fauquet-Alekhine P, Pahuet N, Labrucherie M, Maridonneau C, Geeraerts T, Trabold F, et al., ed. Améliorer la pratique professionnelle par la simulation. Toulouse: Octares; 2011.

95. Nyssen AS. Simulateurs dans le domaine de l'anesthésie. Etudes et réflexions sur les notions de validité et de fidélité. In: Pastré $P$, ed. Apprendre par la simulation. De l'analyse du travail aux apprentissages professionnels. Toulouse: Octares; 2005. p. 269-83.

96. Mollo V, Falzon P. Auto- and allo-confrontation as tools for reflective activities. Appl Ergon 2004;35(6):531-40.

97. Agency for Healthcare Research and Quality, Hopkins J. Effectiveness of continuing medical education. Evidence report technology assessment n¹49. Rockville: AHRQ; 2007.

http://www.ahrq.gov/downloads/pub/evidence/pdf/ 
cme/cme.pdf

98. Haque S, Srinivasan S. A meta-analysis of the training effectiveness of virtual reality surgical simulators. IEEE Trans Inf Technol Biomed 2006;10(1):51-8.

99. Hmelo CE. Computer-assisted instruction in health professions education: a review of the published literature. J Educ Technol Systems 1990;18(2):83-101.

100. Gerson LB, Van Dam J. Technology review: the use of simulators for training in Gl endoscopy. Gastrointest Endosc 2004;60(6):992-1001.

101. Gaffan J, Dacre J, Jones A. Educating undergraduate medical students about oncology: a literature review. J Clin Oncol 2006;24(12):1932-9.

102. Goldwater SH, Milkovich G, Morrison AJ, Lindgren B. Comparison of therapeutic interchange with standard educational tools for influencing fluoroquinolone prescribing. Am J Health Syst Pharm 2001;58(18):1740-5.

103. Ravert P. An integrative review of computerbased simulation in the education process. Comput Inform Nurs 2002;20(5):203-8.

104. McGaghie WC, Issenberg SB, Petrusa ER, Scalese RJ. A critical review of simulation-based medical education research: 2003-2009. Med Educ 2010;44(1):50-63.

105. Salas E, Klein C, King $H$, Salisbury $M$, Augenstein JS, Birnbach DJ, et al. Debriefing medical teams: 12 evidence-based best practices and tips. Jt Comm J Qual Patient Saf 2008;34(9):518-27.

106. Fanning RM, Gaba DM. The role of debriefing in simulation-based learning. Simul Healthc 2007;2(2):115-25.

107. McGaghie WC. Research opportunities in simulation-based medical education using deliberate practice. Acad Emerg Med 2008;15(11):995-1001.

108. Edelson DP, Litzinger B, Arora V, Walsh D, Kim $S$, Lauderdale DS, et al. Improving in-hospital cardiac arrest process and outcomes with performance debriefing. Arch Intern Med
2008;168(10):1063-9.

109. Wayne DB, Barsuk JH, O'Leary KJ, Fudala MJ, McGaghie WC. Mastery learning of thoracentesis skills by internal medicine residents using simulation technology and deliberate practice. J Hosp Med 2008;3(1):48-54.

110. Wayne DB, Didwania A, Feinglass J, Fudala MJ, Barsuk JH, McGaghie WC. Simulation-based education improves quality of care during cardiac arrest team responses at an academic teaching hospital: a case-control study. Chest 2008;133(1):56-61.

111. Barsuk JH, Ahya SN, Cohen ER, McGaghie WC, Wayne DB. Mastery learning of temporary hemodialysis catheter insertion by nephrology fellows using simulation technology and deliberate practice. Am J Kidney Dis 2009;54(1):70-6.

112. Barsuk JH, McGaghie WC, Cohen ER, Balachandran JS, Wayne DB. Use of simulationbased mastery learning to improve the quality of central venous catheter placement in a medical intensive care unit. J Hosp Med 2009;4(7):397-403.

113. Pugh CM, Domont ZB, Salud LH, Blossfield $\mathrm{KM}$. A simulation-based assessment of clinical breast examination technique: do patient and clinician factors affect clinician approach? Am J Surg 2008;195(6):874-80.

114. Kneebone R. Perspective: Simulation and transformational change: the paradox of expertise. Acad Med 2009;84(7):954-7.

115. Kneebone RL, Kidd J, Nestel D, Barnet A, Lo $B$, King R, et al. Blurring the boundaries: scenariobased simulation in a clinical setting. Med Educ 2005;39(6):580-7.

116. Murray DJ, Boulet JR, Avidan M, Kras JF, Henrichs B, Woodhouse J, et al. Performance of residents and anesthesiologists in a simulationbased skill assessment. Anesthesiology 2007;107(5):705-13.

117. American College of Chest Physicians, McGaghie WC, Siddall VJ, Mazmanian PE, Myers J. Lessons for continuing medical education from simulation research in undergraduate and graduate medical education: effectiveness of continuing medical education. Chest 2009;135(3 Suppl): 
62S-8S.

118. Seymour NE. VR to OR: a review of the evidence that virtual reality simulation improves operating room performance. World $\mathrm{J}$ Surg 2008;32(2):182-8.

119. Salas E, DiazGranados D, Weaver SJ, King H. Does team training work? Principles for health care. Acad Emerg Med 2008;15(11):1002-9.

120. Berkenstadt H, Ziv A, Gafni N, Sidi A. Incorporating simulation-based objective structured clinical examination into the Israeli National Board Examination in Anesthesiology. Anesth Analg 2006;102(3):853-8.

121. Berkenstadt H, Ziv A, Gafni N, Sidi A. The validation process of incorporating simulationbased accreditation into the anesthesiology Israeli national board exams. Isr Med Assoc J 2006;8(10):728-33.

122. Weller J, Morris R, Watterson L, Garden A, Flanagan B, Robinson B, et al. Effective management of anaesthetic crises: development and evaluation of a college-accredited simulationbased course for anaesthesia education in Australia and New Zealand. Simul Healthc 2006;1(4):209-14.

123. Vollmer J, Monk S, Heinrichs W. Staff education for simulation: train-the-trainer concepts. In: Kyle RR, Murray WB, ed. Clinical simulation: operations, engineering, and management. Burlington: Academie Press; 2008. p. 625-42.

124. Chow RE, Naik VN. Experiential training for new simulation coordinators. In: Kyle RR, Murray WB, ed. Clinical simulation: operations, engineering, and management. Burlington: Academic Press; 2008. p. 643-6.

125. Inspection générale des affaires sociales, Bras L, Duhamel G. Formation médicale continue et évaluation des pratiques professionnelles des médecins. Paris: IGAS; 2008.

http://lesrapports.ladocumentationfrancaise.fr/BRP/ 084000751/0000.pdf

126. Kirkpatrick DL, Kirkpatrick JD. Evaluating training programs: the four levels. San Francisco: Berrett-Koehler Publishers; 1998.

127. Kirkpatrick D. Great ideas revisited. Techniques for evaluating training programs. Revisiting Kirkpatrick's four-level model. Training
Dev 1996;50(1):54-9.

128. Haute Autorité de Santé. Élaboration de critères de qualité pour l'évaluation et l'amélioration des pratiques professionnelle. Guide méthodologique. Saint-Denis La Plaine: HAS; 2007. http://www.has-sante.fr/portail/upload/docs/ application/pdf/criteres_de_qualite_pour_levaluatio n_et_lamelioration_de.pdf

129. Michel P, Minodier C, Lathelize M, MotyMonnereau C, Domecq S, Chaleux M, et al. Les événements indésirables graves associés aux soins observés dans les établissements de santé. Résultats des enquêtes nationales menées en 2009 et 2004. Doss Solidarité Santé 2010;(17).

130. Moll MC, Granry JC. Simulation et DPC : les RMM simulés. Rev Samu 2010;32(n Spécial).

131. Stoschek F. Les pratiques de l'intervenant, performance humaine EDF; Les règles de la sécurité de fonctionnement en aéronautique. In: Journées des grandes écoles de la mer. Colloque "Facteurs humains et sécurité maritime". Havre 26 et 27 janvier 2011. Brest: Association française des capitaines de navires; 2011.

132. Roussel P, Moll MC, Guez P. Méthodes et outils essentiels de la gestion des risques en santé. Étape 2 : identifier les risques à priori. Risque Qual Milieu Soins 2007;4(4).

133. Howard SK, Gaba DM, Fish KJ, Yang G, Sarnquist $\mathrm{FH}$. Anesthesia crisis resource management training: teaching anesthesiologists to handle critical incidents. Aviat Space Environ Med 1992;63(9):763-70.

134. Marsch SC, Muller C, Marquardt K, Conrad G, Tschan F, Hunziker PR. Human factors affect the quality of cardiopulmonary resuscitation in simulated cardiac arrests. Resuscitation 2004;60(1):51-6.

135. Pittman J, Turner B, Gabbott DA. Communication between members of the cardiac arrest team: a postal survey. Resuscitation 2001;49(2):175-7.

136. Brindley PG, Reynolds SF. Improving verbal communication in critical care medicine. J Crit Care 2011;26(2):155-9.

137. Cooper S, Wakelam A. Leadership of resuscitation teams: "Lighthouse Leadership". 
Resuscitation 1999;42(1):27-45.

138. Harrison TK, Manser T, Howard SK, Gaba DM. Use of cognitive aids in a simulated anesthetic crisis. Anesth Analg 2006;103(3):551-6.

139. Haute Autorité de Santé. CD-Rom Audit clinique ciblé (ACC) 2007. Saint-Denis La Plaine: HAS; 2007.

http://www.has-sante.fr/portail/jcms/c_746701/cdrom-audit-clinique-cible-acc-2007

140. Haute Autorité de Santé. Revue de mortalité et de morbidité (RMM). Guide méthodologique. Saint-Denis La Plaine: HAS; 2009.

http://www.has-sante.fr/portail/upload/docs/ application/pdf/2009-08/guide_rmm_juin_09.pdf

141. Haute Autorité de Santé. Chemin clinique. Une méthode d'amélioration de la qualité. Saint-Denis La Plaine: HAS; 2005.

http://www.has-sante.fr/portail/upload/docs/ application/pdf/2009-08/chemin_clinique_4.pdf

142. Gale TC, Roberts MJ, Sice PJ, Langton JA, Patterson FC, Carr AS, et al. Predictive validity of a selection centre testing non-technical skills for recruitment to training in anaesthesia. $\mathrm{Br} \mathrm{J}$ Anaesth 2010;105(5):603-9.

143. Kurrek MM, Fish KJ. Anaesthesia crisis resource management training: an intimidating concept, a rewarding experience. Can J Anaesth 1996;43(5 Pt 1):430-4.

144. Devitt JH, Kurrek MM, Cohen MM, CleaveHogg D. The validity of performance assessments using simulation. Anesthesiology 2001;95(1):36-42.

145. Weller J, Wilson L, Robinson B. Survey of change in practice following simulation-based training in crisis management. Anaesthesia 2003;58(5):471-3.

146. Abella BS, Alvarado JP, Myklebust $\mathrm{H}$, Edelson DP, Barry A, O'Hearn N, et al. Quality of cardiopulmonary resuscitation during in-hospital cardiac arrest. JAMA 2005;293(3):305-10.

147. Naik VN, Matsumoto ED, Houston PL, Hamstra SJ, Yeung RY, Mallon JS, et al. Fiberoptic orotracheal intubation on anesthetized patients: do manipulation skills learned on a simple model transfer into the operating room? Anesthesiology 2001;95(2):343-8.

148. Barsuk D, Ziv A, Lin G, Blumenfeld A, Rubin O, Keidan I, et al. Using advanced simulation for recognition and correction of gaps in airway and breathing management skills in prehospital trauma care. Anesth Analg 2005;100(3):803-9, table.

149. Schwid HA, Rooke GA, Ross BK, Sivarajan M. Use of a computerized advanced cardiac life support simulator improves retention of advanced cardiac life support guidelines better than a textbook review. Crit Care Med 1999;27(4):821-4.

150. Abraham J, Wade DM, O'Connell KA, Desharnais S, Jacoby R. The use of simulation training in teaching health care quality and safety: an annotated bibliography. Am J Med Qual 2011;26(3):229-38.

151. Andersen PO, Jensen MK, Lippert A, Ostergaard D. Identifying non-technical skills and barriers for improvement of teamwork in cardiac arrest teams. Resuscitation 2010;81(6):695-702.

152. Cook DA, Hatala R, Brydges $R$, Zendejas $B$, Szostek JH, Wang AT, et al. Technology-enhanced simulation for health professions education: a systematic review and meta-analysis. JAMA 2011;306(9):978-88.

153. Gouvistos F, Vallet B, Scherpereel P. Les simulateurs d'anesthésie: intérêts et limites à travers l'expérience de plusieurs centres universitaires européens. Ann Fr Anesth Réanim 
1999;18(7):787-95.

154. McIntosh C, Macario A, Flanagan B, Gaba D. Simulation: what does it really cost? [poster presentation]. In: Society for Medical Simulation, ed. 6th International meeting on medical simulation. January 14-17 2006, San Diego, CA, USA. Santa Fé: SMS; 2006.

155. Savoldelli GL, Naik VN, Hamstra SJ, Morgan PJ. Barriers to use of simulation-based education. Can J Anaesth 2005;52(9):944-50.

156. Botden SM, Buzink SN, Schijven MP, Jakimowicz JJ. Augmented versus virtual reality laparoscopic simulation: what is the difference? A comparison of the ProMIS augmented reality laparoscopic simulator versus LapSim virtual reality laparoscopic simulator. World J Surg 2007;31(4):764-72. 
